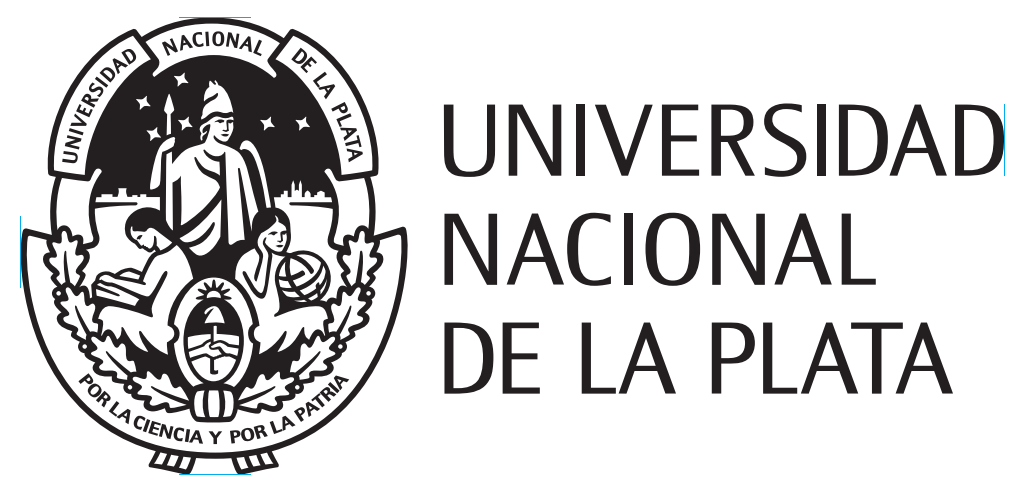

Facultad de Ciencias Astronómicas y Geofísicas

\title{
Modelado de efectos de carga en la Patagonia austral
}

Geof. Eric Rodolfo Marderwald

Director: Dr.-ing. habil. Andreas Jörg Richter

Codirector: Lic. Raúl Aníbal Perdomo 



\section{Agradecimientos}

Quiero expresar mi gratitud a mis directores, el Dr.-Ing. Andreas J. Richter y el Lic. Raúl A. Perdomo por su permanente esfuerzo y dedicación con que han dirigido mi trabajo doctoral. Su ayuda y consejo han sido fundamentales para el desarrollo y culminación de esta tesis.

Deseo agradecer a la Facultad de Ciencias Astronómicas y Geofísicas por el apoyo y los medios recibidos durante estos años de mi progreso académico. Debo un especial reconocimiento al Consejo Nacional de Investigaciones Científicas y Técnicas (CONICET) y al Servicio Alemán de Intercambio Académico (DAAD), organismos que han hecho posible mediante la concesión de becas de estudio doctorales el llevar a cabo esta tarea.

De igual modo, me gustaría agradecer a los miembros del Institut für Planetare Geodäsie de la Technische Universität Dresden, Alemania, principalmente al Prof. Martin Horwath, el Dr. Andreas Groh, el Dr. Mirko Scheinert y el Prof. Reinhard Dietrich por sus inestimables consejos, guía y colaboración durante mis estadías en Alemania.

A lo largo de estos años he contado con la ayuda inestimable y el estímulo de profesores, investigadores, compañeros, amigos y personal administrativo, deseo expresar mi reconocimiento a cada uno de ellos. Junto a ellos agradezco al jurado examinador de esta tesis por sus valiosas sugerencias.

Hago extensivo estos agradecimientos a los organismos que han proporcionado datos y observaciones (mareográficos, de altimetría por satélite, modelos digitales del terreno, meteorológicos, etc.) imprescindibles para este trabajo y que se citan en esta memoria.

Finalmente, deseo dar las gracias a mi familia, sin su apoyo continuo e incondicional no habría sido posible la realización de esta tesis. 


\section{Resumen}

Los Campos de Hielo Patagónico, las masas de hielo extrapolar mas grandes en el hemisferio sur, se encuentran afectadas por una compleja estructura tectónico-reológica, un intenso ajuste glacio-isostático y un rápido retroceso glaciar. El trabajo de investigación desarrollado tiene, como objetivo general, el avance del conocimiento de los procesos geodinámicos en la región de la Patagonia austral en base al modelado de efectos de la respuesta elástica a cambios de masa superficial: deformación elástica de la corteza, cambios en el campo gravitatorio, deformación y desplazamiento de las superficies equipotenciales, inclinaciones de las superficie terrestre y equipotenciales. Este proyecto incluye el desarrollo de herramientas de cálculo; la compilación de modelos regionales de carga; la integración, interpretación y síntesis de los resultados del modelado. Estos modelados comprenden la respuesta elástica a los cambios actuales de la masa de hielo en el sur de la Patagonia; los efectos de la carga elástica derivados de distintos escenarios de carga hidrológica, incluyendo las rupturas del endicamiento de los brazos Rico y Sur del lago Argentino producto del avance del glacial Perito Moreno y las previstas represas del Río Santa Cruz. Así mismo, se presentan también resultados para la carga oceánica, su uso para la validación de los cálculos y carga atmosférica. Los efectos modelados se integran en modelos regionales, que posibilitan la comparación con observaciones geodésicas y geofísicas. De esta manera se obtiene nueva información sobre las propiedades reológicas de la corteza y se aporta al conocimiento de los procesos geodinámicos efectivos en la región. Estos resultados se evalúan con respecto a los observables geodésicos y su potencial para validar o acotar modelos de tierra elástica. Se discute las consecuencias para la determinación de las tasas de deformación precisas, el alcance para nuestra comprensión del intenso levantamiento observado en el Campo de Hielo Patagónico Sur, y los requisitos y perspectivas de una observación geodésica de los efectos de carga elásticos para el conocimiento de partes de la estructura terrestre. Por lo que los resultados contribuyen a la explicación causal de efectos observados por distintos métodos y permiten recomendaciones concretas para futuros estudios de determinados efectos de carga u otros efectos geodinámicos. 


\section{Índice general}

Agradecimientos $\quad$ I

Resumen

1. Introducción 1

2. Efectos de cargas superficiales y su modelado: bases teóricas $\quad 7$

2.1. Carga superficial, mecanismo y efectos . . . . . . . . . . . . 7

2.2. Respuesta elástica vs. visco-elástica (modelos semi-espacio elástico vs. Max-

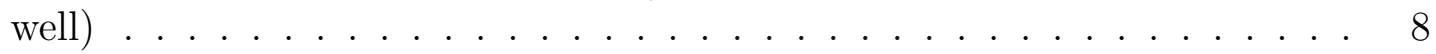

2.3. Modelado mediante funciones de Green . . . . . . . . . . . . . . . . . . 9

2.3.1. Ecuaciones que rigen la deformación . . . . . . . . . . . . . 9

2.3.2. Números de Love . . . . . . . . . . . . . . . . . . . . . . . 11

2.3.3. Funciones de Green . . . . . . . . . . . . . . . . . . . 12

2.3.4. Integral de convolución, cálculo de efectos de carga . . . . . . . . . 13

2.4. Modelos de carga . . . . . . . . . . . . . . . . . . . . 14

2.5. Modelos de Tierra . . . . . . . . . . . . . . . . . . . 14

2.6. Código ELOADE . . . . . . . . . . . . . . . . . . . . . 15

3. Patagonia austral: sinopsis geofísica-geomorfológica-geográfica 19

4. Las Cargas 25

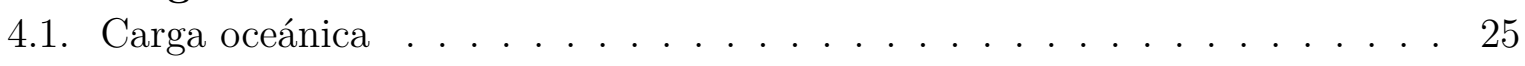

4.2. Carga atmosférica . . . . . . . . . . . . . . . . 27

4.3. Carga hidrológica: hidrología continental . . . . . . . . . . . . . . 31

4.3.1. Los grandes lagos patagónicos . . . . . . . . . . . . . . . . . . . . . . . . . . . 31

4.3.2. Brazos Rico y Sur . . . . . . . . . . . . . . . . . . . . . . . . . . . . . . . . . . . 32

4.3.3. Represas del Río Santa Cruz . . . . . . . . . . . . . . . . . . 34

4.4. Carga glaciar . . . . . . . . . . . . . . . . . . . 37

5. Resultados $\quad 41$

5.1. Efectos de carga oceánica . . . . . . . . . . . . . . . . . 42

5.1.1. Predicciones por Bos and Scherneck (2011) - Modelo EOT11a . . . 42

5.1.2. Predicciones - ELOADE, modelo EOT11a . . . . . . . . . . . 45

5.1.3. Modelo Regionalizado: EOT11a más D'onofrio et al. (2016) . . . . . 47

5.1.4. Efectos de carga oceánica no-mareal . . . . . . . . . . . . . . . . . 49

5.2. Efectos de carga atmosférica . . . . . . . . . . . . . . . . 49 
5.2.1. Ciclo anual y diurno . . . . . . . . . . . . . . . . . . . 49

5.2.2. Evento de cambio abrupto de presión atmosférica . . . . . . . . . . 49

5.3. Efectos de carga hidrológica . . . . . . . . . . . . . . . . . . 52

5.3.1. Los grandes lagos patagónicos . . . . . . . . . . . . . . 52

5.3.2. Brazo Rico/Sur . . . . . . . . . . . . . . . . . . . 55

5.3.3. Represas del Río Santa Cruz . . . . . . . . . . . . . . . . . . 58

5.4. Efectos de carga glaciar . . . . . . . . . . . . . . . . 64

$\begin{array}{ll}\text { 6. Integración y aplicaciones } & 67\end{array}$

6.1. Integración espectral . . . . . . . . . . . . . . . . 68

6.2. Integración local $\ldots \ldots \ldots \ldots$. . . . . . . . . . . . . . 69

$\begin{array}{ll}\text { 7. Conclusiones } & 73\end{array}$

$\begin{array}{ll}\text { Índice figuras } & 75\end{array}$

$\begin{array}{ll}\text { Índice tablas } & 77\end{array}$

$\begin{array}{ll}\text { Bibliografía } & 77\end{array}$ 


\section{Capítulo 1}

\section{Introducción}

La Patagonia austral, región geográfica ubicada entre los países de Argentina y Chile, se extiende en latitud desde la mitad del Golfo de San Jorge hasta el punto mas austral del archipiélago de Tierra del Fuego, el Cabo de Hornos (ver mapa, figura 1.1). Esta se destaca, por un lado, por la acumulación de masa de hielo templado más grande del hemisferio sur (Casassa et al., 2014), que responde muy sensiblemente a cambios climáticos (Mercer, 1976; Strelin et al., 2011; F. et al., 2011; Strelin et al., 2014), los Campos de Hielo Patagónico Norte y Sur. Por otro lado, a una situación tectónica-reológica particular, determinada por la subducción de una dorsal oceánica activa a la altura de la península Taitao, el punto Triple de Chile (Bird, 2003; Russo et al., 2010), la apertura de la Ventana Astenosférica Patagónica (Gorring and Kay, 2001; Breitsprecher and Thorkelson, 2009), el ascenso de material caliente y de baja viscocidad del manto debajo de los Campos de Hielo Patagónico (Lin, 2014; Russo et al., 2010) y el movimiento transformante entre las placas de Sudamérica y Scotia en Tierra del Fuego (Mendoza et al., 2015).

En la superficie de la Tierra ocurren continuamente cambios y desplazamientos de masas. La tierra sólida responde a estas cargas en la superficie con una deformación y cambios del campo gravitatorio. La magnitud de los efectos de la carga depende, por un lado, de la masa, la distancia y el tiempo de la carga y, por otro lado, de las propiedades reológicas de la tierra sólida. Los efectos de carga pueden modelarse, lo que requiere la introducción de un modelo de tierra, que describe las propiedades mecánicas de la Tierra, y un modelo de carga, que describe la distribución de la masa de la carga en el espacio y el tiempo. En el enfoque clásico, los efectos de carga se predicen como la suma de dos contribuciones, que se modelan por separado: en primer lugar, una respuesta puramente elástica y, en segundo lugar, una respuesta visco-elástica gravitacional con escalas de tiempo climáticas. Para la predicción de la respuesta elástica se utiliza un modelo de tierra elástico. Normalmente se aplican modelos terrestres de simetría radial, asumiendo propiedades elásticas homogéneas en todo el mundo. Estos modelos se derivan de información sismológica, donde se supone que el comportamiento medio en la escala de tiempo de las ondas sísmicas es representativo de la respuesta instantánea a cualquier fuerza de carga superficial. El Ajuste Glacio-Isostático (AGI) debido a los cambios de la masa de hielo impulsados por el clima es el ejemplo clásico de la respuesta visco-elástica. Los modelos 


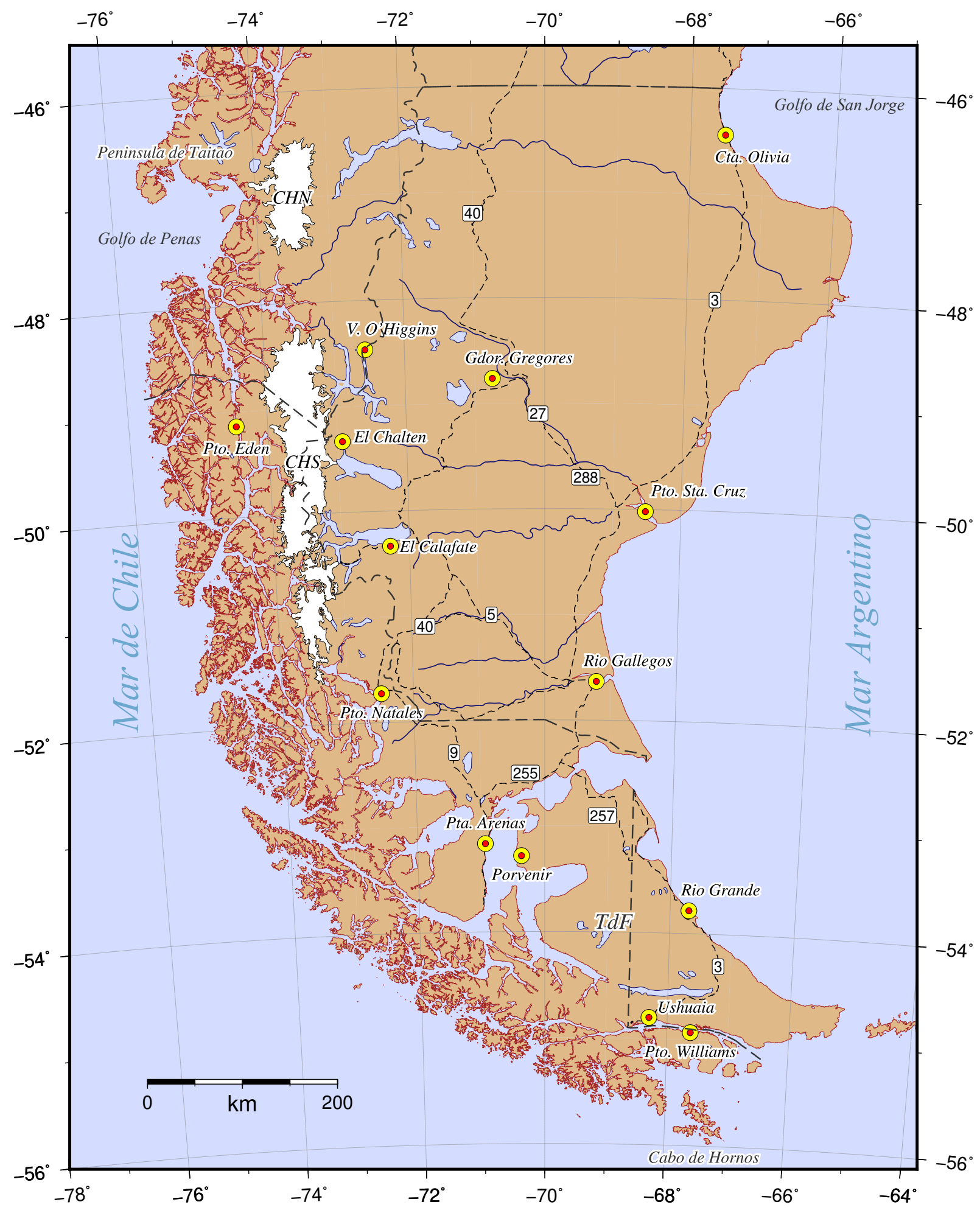

Figura 1.1: Mapa correspondiente a la Patagonia austral. Se muestran algunos de los principales centros urbanos, así también rutas nacionales y provinciales de Argentina y Chile. Campo de Hielo Norte (CHN), Campo de Hielo Sur (CHS) e Isla Grande de Tierra del Fuego (TdF). 
de AGI aplican un modelo terrestre visco-elástico, que describe la magnitud de los efectos no sólo en función de la masa y la distancia de la carga, sino también en función del tiempo transcurrido desde la aplicación de la carga. Normalmente se utiliza un modelo de Maxwell (Whitehouse, 2018). Dado que las propiedades visco-elásticas de la Tierra varían sustancialmente en todo el mundo, se aplican parámetros de modelos terrestres regionales individuales. Pero dentro de una región (dominio del modelo), las propiedades visco-elásticas se asumen generalmente como lateralmente homogéneas. Limites sobre los valores de los parámetros de los modelos regionales de tierra visco-elásticos pueden determinarse de las observaciones geodésicas de los efectos de ajuste glacio-isostático (por ejemplo, Milne et al. (2001); Larsen et al. (2005); Hu and Freymueller (2019)).

Los Campos de Hielo Patagónico, se extienden entre los 46,4 y 51,6 latitud Sur a lo largo de los Andes Patagónicos. Estos actualmente se encuentran sujetos a una rápida pérdida de masa de hielo (Malz et al., 2018; Braun et al., 2019; Richter et al., 2019; Dussaillant et al., 2019). Ubicados en una región donde la reología está regida por un peculiar entorno tectónico. La muy baja viscosidad del manto (1,8-8 x $1018 \mathrm{~Pa}$ s; Lange et al. (2014)) y la litosfera relativamente fina $(36,5 \mathrm{~km}$; Lange et al. (2014)) implican tiempos de relajación visco-elástica mucho más cortos que en otras regiones afectadas por el AGI. Además, los modelos sugieren una modulación significativa del patrón de intensidad del AGI por las heterogeneidades laterales impuestas por la configuración geométrica de las placas subductadas y la circulación del manto a través de la ventana astenosferica (Klemann et al., 2007). Las observaciones realizadas con los Global Navigation Satellite System (GNSS) en la región del Campo de Hielo Patagónico Sur revelan un intenso levantamiento con un patrón concéntrico en forma de cúpula (Dietrich et al., 2010a; Lange et al., 2014; Richter et al., 2016). Con una tasa máxima de $4 \mathrm{~cm} / \mathrm{a}$, ubicada en la parte septentrional del Campo de Hielo Sur, es una de las tasas de levantamiento más rápidas notificadas en todo el mundo (por ejemplo, Barletta et al. (2018); Larsen et al. (2005); Dietrich et al. (2005)). Los modelos regionales de AGI (Ivins and James, 1999, 2004; Klemann et al., 2007; Lange et al., 2014) explican este levantamiento como respuesta a los cambios en la masa de hielo desde el máximo alcanzado durante la Pequeña Edad de Hielo (AD 1870, Lange et al. (2014)). La contribución elástica puede alcanzar la mitad del levantamiento total en las proximidades de los glaciares con rápido retroceso del Campo de Hielo Patagónico Sur (Lange et al., 2014). Lange et al. (2014) utilizan las tasas de levantamiento observadas por GNSS para ajustar los parámetros del modelo terrestre visco-elástico. Sin embargo, las velocidades de levantamiento por sí solas no pueden resolver completamente las ambigüedades entre los modelos terrestres y la historia de carga de hielo. Persisten incertidumbres sustanciales con respecto al historial de la carga de hielo posterior al Pequeña Edad de Hielo, así como en la configuración y la elección de los parámetros de los modelos reológicos.

El motivo más importante se presenta ante la incertidumbre sobre la dependencia temporal de los efectos de la carga. Las cargas, es decir, las masas distribuidas sobre la superficie terrestre que cambian continuamente, ¿en qué proporciones, y a través de qué mecanismo, contribuyen las diferentes escalas de tiempo de los ciclos de carga y descarga a la respuesta total observable?. De hecho, en los períodos de marea se observa una transición de una respuesta elástica pura a una respuesta anelástica (Bos et al., 2015). Esto pone en duda la práctica común de aplicar modelos terrestres puramente 
elásticos para predecir los efectos de la carga a corto plazo y, más en general, la tradicional separación discreta entre la contribución elástica instantánea y la contribución viscoelástica a largo plazo. Además, cada vez se encuentra un mayor cuestionamiento sobre la idoneidad de una tradicional reología maxwelliana en la modelización del AGI (Bos et al., 2015; Ivins and Sammis, 1996). Los resultados de las observaciones realizadas en diferentes partes del planeta sugieren que la respuesta visco-elástica a las cargas superficiales se describe mejor mediante modelos reológicos alternativos como, por ejemplo, el modelo Burgers (Ivins and Sammis, 1996). En este contexto, y en vista de la particular estructura reológica, las inconsistencias entre las predicciones de los modelos clásicos y los efectos de las cargas observadas no sorprenderían en la Patagonia austral. De hecho, las mareas lacustres observadas en el Lago Fagnano en Tierra del Fuego, a unos $500 \mathrm{~km}$ al sur de Campo de Hielo Sur, han revelado una amplificación del efecto de la carga de las mareas oceánicas de entre 20 a $30 \%$ en relación con las predicciones de los modelos elásticos convencionales (Richter et al., 2009). La validación regional de los modelos de mareas oceánicas basados en registros de mareas de presión en la plataforma atlántica fueguina (Richter et al., 2012a) permitió descartar las deficiencias del modelo de carga aplicado como causa de la inconsistencia entre los efectos de la carga de mareas oceánicas modelizada y la observada. Esto llevó a la conclusión de una anomalía regional en las propiedades elásticas. Se ha señalado que esta anomalía está relacionada con el sistema de fallas transformante Magallanes-Fagnano (Richter et al., 2009), pero también podría ser causada por la Ventana Astenosférica Patagónica. Si en efecto está relacionada con la ventana astenosférica, la anomalía descubierta en el Lago Fagnano tendría consecuencias para la respuesta a los cambios actuales de la masa de hielo en el Campo de Hielo Sur y la modelización del AGI en la Patagonia austral. Por otro lado, en el Lago Argentino ubicado en la margen oriental de la Cordillera de los Andes, cubriendo $1330 \mathrm{~km} 2$ entre 50.0-50.5 ${ }^{\circ} \mathrm{S}$ y $72.0-73.3^{\circ} \mathrm{W}$, es alimentado por varios glaciares que descienden del Campo de Hielo Sur. Las observaciones de la marea lacustre han revelado un cambio de fase en la respuesta de carga de las mareas oceánicas en comparación con las predicciones del modelo (Richter et al., 2016). Esta inconsistencia de fase puede ser el resultado de deficiencias locales de los modelos globales de mareas oceánicas y no permite sacar conclusiones sobre la validez del modelado convencional de los efectos de la carga elástica en la región.

El presente trabajo tiene como objetivo contribuir a la resolución de estas ambigüedades a través del desarrollo de una herramienta computacional operativa y su aplicación para el cálculo de efectos de la respuesta elástica de la tierra sólida a cargas superficiales. De esta manera aporta al entendimiento del ajuste glacio-isostático en la Patagonia austral y del comportamiento visco-elástico de la tierra sólida en general, a la explicación causal de las observaciones geodésicas y geofísicas, y a la reconstrucción de la evolución glaciar y de cambios climáticos en la zona de estudio. Un modelo preciso del ajuste glacio-isostático también es un requisito fundamental para la cuantificación de la pérdida de masa actual a partir de datos de gravimetría satelital, como los provistos por las misiones Gravity Recovery and Climate Experiment (GRACE) y GRACE-Follow On (GRACE-FO) (Richter et al., 2019). Los cambios de masa de hielo, a su vez, afectan de manera directa reservas de recursos hídricos, el nivel del mar y el ecosistema. Mejoras en los modelos existentes de efectos del ajuste glacio-isostático y de la respuesta elástica a señales de carga actuales ayudan de esta manera a desarrollar estrategias de monitoreo y mitigación de 
consecuencias del cambio climático. Por otro lado, la validación de los modelos reológicos de la tierra mediante una comparación de observaciones geodésicas de efectos de carga con predicciones de un modelado geofísico contribuye a la evaluación del riesgo sísmico en esta región de compleja tectónica. Motivo de este trabajo es también aportar al crecimiento de la estructura geodésica provincial, tanto en Tierra del Fuego como en Santa Cruz, buscando mejorar las redes gravimétricas y altimetrías, determinando los posibles efectos sobre estas.

Esta tesis se estructura de la siguiente manera: El siguiente capítulo resume las bases teóricas del modelado de la respuesta elástica de la Tierra a cargas superficiales. El mismo culmina con la descripción breve del código ELOADE desarrollado en el marco de este trabajo. El tercer capítulo comprende una descripción de la región de estudio, la Patagonia austral, su geomorfología y características climáticas más significativas. Seguido se presenta el cuarto capítulo que presenta la descripción de las cargas consideradas y su discretización en modelos de carga, a saber: la carga oceánica, atmosférica, hidrológica y glaciar. El quinto capítulo resume los resultados del cálculo de una serie de efectos generados por estas cargas obtenidos mediante el código desarrollado y una validación del mismo. El capítulo sexto resume los resultados obtenidos en forma espectral y local, proporcionando información conceptual y cuantitativa acerca de la magnitud de los efectos las cargas combinadas. La tesis concluye con un resumen de las principales conclusiones y proyecciones futuras. 


\section{Capítulo 2}

\section{Efectos de cargas superficiales y su modelado: bases teóricas}

En este Capítulo se revisan algunos conceptos y desarrollos teóricos fundamentales que serán utilizados en el estudio de la respuesta de la Tierra sólida a las distintas clases de cargas superficiales como, los océanos, la atmósfera, la hidrología continental y los glaciares. Se recopilarán las ecuaciones elementales que rigen las deformaciones elásticas de la Tierra sólida, originadas por las fuerzas que derivan de un potencial perturbador, considerando diversos modelos terrestres. Finalmente se extienden las bases teóricas al desarrollo de un software que permite la evaluación de estos modelos y obtener valores estimados de la respuesta elástica de la Tierra a cargas superficiales, por lo que se explica brevemente el funcionamiento y uso.

\subsection{Definición de carga superficial, mecanismo bási- co y efectos fundamentales}

Entendemos por carga superficial a una masa dispuesta sobre la superficie terrestre. Un cambio de masa actúa sobre la superficie terrestre mediante una fuerza superficial, un potencial de carga y un potencial de deformación, ambos componen el potencial perturbador. El primero es el potencial generado por los cambios de masas (o masa aplicada) el cual aplica la fuerza y el segundo generado por la propia deformación, la cual tiene la capacidad de almacenar energía potencial elástica (memoria a la deformación). Los efectos fundamentales son deformación del cuerpo terrestre y cambios de su campo gravitatorio. A partir de estos se generan una serie de efectos observables como: desplazamientos de puntos de observación geométrica en la superficie terrestre, deformación y desplazamiento de las superficies equipotenciales, cambio de la gravedad, inclinaciones de la superficie terrestre y equipotenciales y cambios relativos del nivel del mar, entre otros (Lambeck, 1988). Las magnitudes de estos efectos en un determinado punto dependen de la masa, la distancia de la carga y las propiedades elásticas de la litosfera. 
En el marco de este trabajo nos concentramos en los cuatro agentes principales del sistema terrestre que generan efectos de carga: hidrosfera continental, océano, atmósfera y criósfera. De esta manera se entiende que, los modelos de carga representan, cambios recientes de la masa glaciar, mareas oceánicas, cambios en la hidrología continental y variaciones de la presión atmosférica.

\subsection{Respuesta elástica vs. visco-elástica (modelos semi- espacio elástico vs. Maxwell)}

Cuando se coloca una carga en la superficie terrestre, la Tierra responde inicialmente de forma elástica, con subsidencia cerca de la carga y pequeños levantamientos en la lejanía. Si la carga se mantiene fija, la Tierra (asunción más simple es un sólido de Maxwell) continúa deformándose viscoelásticamente, acercándose a un límite de compensación casi isostática.

Cuando un cuerpo sólido es sometido a una fuerza (carga, esfuerzo o tensión) se produce en él una deformación. Si al cesar la fuerza aplicada el material vuelve a su estado inicial y de manera instantánea, diremos que ha experimentado una deformación elástica. La deformación es reversible y no permanente. Los limites a los cuales un cuerpo puede deformarse elásticamente depende de las propiedades reológicas del mismo. Sobre la superficie terrestre esta respuesta se encuentra determinada principalmente por la elasticidad de la litosfera, donde la deformación depende de cada punto e instante en que se observa.

Si la carga persiste por un período extenso, se suma a la respuesta elástica de la litosfera un ajuste isostático a través del desplazamiento paulatino del material viscoso del manto (Peltier, 1998). Este proceso perdura por cientos y/o miles de años con intensidad decreciente. La reacción viscosa se combina con los mismos efectos como la respuesta elástica (deformación cortical y de superficies equipotenciales, inclinaciones, cambios relativos del mar, etc.). La magnitud de estos efectos depende de la masa, la distancia a la carga, el tiempo transcurrido desde la aplicación de la carga y de las propiedades reológicas de la corteza y manto superior (Scherneck, 1990; Ivins and James, 1999).

En contraste con los efectos de carga oceánica, los efectos de carga glaciar causan un flujo en el manto. Para la descripción de tal fenómeno se requiere un estudio mucho más complicado que las ecuaciones de un cuerpo perfectamente elástico. Un modelo muy común en este caso es un sólido de Maxwell, cuyo comportamiento está bien representado por un elemento elástico en serie con un amortiguador (modelo reológico), lo que comprendería un sólido visco-elastico, en este caso su comportamiento está regido por un fluido newtoniano, su densidad y los parámetros de Lamé (parámetros $\lambda$ y $\mu$, constantes que caracterizan el comportamiento elástico lineal de un sólido isótropo en pequeñas deformaciones). Estos modelos incorporan a la respuesta elástica una dependencia a la velocidad e historia de la deformación, siendo imprescindibles en el modelado de sólidos que poseen comportamientos de fluencia y relajación. 


\subsection{Principio del modelado de efectos elásticos de carga utilizando funciones de Green}

Se describe el modelado de la deformación elástica de un modelo de Tierra esférico, radialmente estratificado y gravitante en respuesta a una carga de masa puntual, superficial e impulsiva. No es menester de este trabajo el presentar un desarrollo exhaustivo de las ecuaciones y procedimientos, dicho esto, se presenta un desarrollo consiso de las ecuaciones, tales procedimientos pueden ser revisados de diferentes fuentes ampliamente citadas a lo largo de este trabajo, como son Pekeris and Jarosch (1958); Longman (1962, 1963); Backus (1967); Farrell (1972); Dahlen et al. (1975); Scherneck (1990); Agnew (1997).

El desarrollo contempla el cálculo de los números de Love partiendo de un modelo de Tierra elástico generalizado, de los cuales se obtienen las funciones de Green como una suma ponderada de estos. Los efectos, desplazamientos, inclinaciones, aceleraciones y deformaciones sobre la superficie terrestre causada por cualquier carga superficial pueden ser calculados resolviendo la integral de convolución entre la carga determinada y las funciones de Green (Farrell, 1972).

\subsubsection{Ecuaciones que rigen la deformación}

La teoría permite el cálculo de efectos elásticos de carga en un punto de la Tierra causado por una masa unitaria, esta respuesta de la Tierra a las fuerzas derivadas de un potencial perturbador se extiende posteriormente a una distribución arbitraria de carga superficial. Se proveen las soluciones para perturbaciones donde la primer aproximación corresponde a un modelo de la Tierra que está en equilibrio mecánico y se encuentra en reposo con respecto a algún marco de referencia inercial. Al limitarnos al efecto elástico y por ende instantáneo, nos independizamos de la rotación de la Tierra. Por tal, el modelo de Tierra no posee un momento angular neto. Suponemos que este modelo de Tierra está compuesto por un continuo aislado, autogravitante, perfectamente elástico (Dahlen et al., 1975) y con cuando los tres parámetros $\lambda, \mu$ y $\rho$ son funciones de la profundidad, siendo este último la densidad volumétrica de la Tierra. Las ecuaciones de equilibrio para la Tierra deformada, en las cuales se toman en cuenta las perturbaciones del campo de gravedad e inicialmente sometida a una tensión hidrostática antes de la aplicación de la carga superficial, son derivadas de las ecuaciones de las oscilaciones libres de la Tierra (ecuaciones de movimiento) dadas por Alterman et al. (1959); Longman (1962).

Partimos de las ecuaciones básicas que rigen la deformación para un modelo de Tierra SNREI (Spherical - NonRotating - Elastic - Isotropic; véase sección 2.5) sometido a un esfuerzo inicial hidrostático y a fuerzas derivadas de un potencial perturbador. Estas son las ecuaciones de conservación de momento (las cuales relacionan las fuerzas aplicadas, ecuación 2.1) y la ecuación de Poisson (satisfecha por el potencial gravitatorio bajo hipótesis de auto gravitación, ecuación 2.2). Donde denotamos por $\rho, \phi, \mathrm{y} \boldsymbol{\tau}$, respectivamente, el campo escalar de densidad de masa, el campo escalar del potencial y el campo tensor de esfuerzo estático inicial de la configuración del equilibrio estático; el tensor de 
esfuerzo estático inicial no es, en general, necesario que sea isotrópico. Estos tres campos están relacionados por la ecuación de Poisson. Siendo, $g_{0}$ la aceleración de la gravedad en ausencia de movimiento, $\boldsymbol{s}$ es el vector desplazamiento y los vectores unitarios $\boldsymbol{e}_{\boldsymbol{r}}$ y $\boldsymbol{e}_{\boldsymbol{\theta}}$ correspondientes a las componentes radial y tangenciales, respectivamente.

$$
\begin{gathered}
\rho \frac{\partial^{2} \boldsymbol{s}}{\partial t^{2}}=\nabla \boldsymbol{\tau}-\nabla\left(\rho g \boldsymbol{s e}_{\boldsymbol{r}}\right)-\rho \nabla \phi+g \nabla(\rho \boldsymbol{s}) \boldsymbol{e}_{\boldsymbol{r}}, \\
\nabla^{2} \phi=-4 \pi G \nabla(\rho \boldsymbol{s}) .
\end{gathered}
$$

Donde, $\phi$ está formado por la perturbación en el potencial gravitacional $\phi_{1}$ (potencial debido a la propia deformación) y el potencial debido a cualquier campo de fuerza gravitacional externo $\phi_{2}$ (potencial de la fuerza aplicada: la carga). Como el campo de fuerzas aplicado proviene de las masas en el exterior de la Tierra, su potencial satisface la ecuación de Laplace dentro de la Tierra y por lo tanto no aparece en forma explícita en las ecuaciones de movimiento. Solo se considera a través de las condiciones de borde sobre la superficie libre (Farrell, 1972).

Las ecuaciones 2.1 y 2.2 se resuelven para un modelo de Tierra de simetría esférica cuyas propiedades dependen solamente de la componente radial $r$. Sobre la superficie el esfuerzo tangencial es nulo y el sistema de ecuaciones se limita a las variables escalares $\boldsymbol{s}_{\boldsymbol{r}}$ $s_{\theta}$ y $\phi$. Desarrollando los desplazamientos y el potencial en armónicos esféricos (Farrell, 1972),

$$
\begin{aligned}
& s(r, \theta)=\sum_{n=0}^{\infty}\left(U_{n}(r) P_{n}(\cos (\theta)) \boldsymbol{e}_{r}+V_{n}(r) \frac{\partial P(\cos (\theta))}{\partial \theta} \boldsymbol{e}_{\theta}\right) \\
& \phi(r, \theta)=\sum_{n=0}^{\infty} \Phi_{n}(r) P_{n}(\cos (\theta)) .
\end{aligned}
$$

Siendo $P_{n}(\cos (\theta))$ los polinomios de Legendre de grado n-ésimo. Para una carga superficial con simetría axial, la solución es independiente de la longitud, por lo tanto solo se desarrolla el orden $m=0$ de las funciones de Legendre. Las funciones $U_{n}, V_{n}$ y $\Phi$ son los coeficientes para las perturbaciones armónicas de grado $n$ en el desplazamiento radial, tangencial y en el potencial gravitatorio, respectivamente. La ley de Hooke proporciona la relación de esfuerzo y deformación, ambos infinitesimales para un cuerpo isótropo. De donde se introducen las variables $\tau_{r r} \mathrm{y} \tau_{r \theta}$, y una tercer variable $q$, relacionada al gradiente del potencial en la dirección radial (Farrell, 1972).

$$
q=\frac{\partial \phi}{\partial r}+\frac{n+1}{r} \phi+4 \pi G \rho \boldsymbol{s e}_{r}
$$

Considerando $T_{r r, n}, T_{r \theta, n}$ y $Q_{n}$ a las componentes de $\tau_{r r}$ y $\tau_{r \theta}$, y $q$ en el desarrollo de armónicos esféricos, respectivamente, las ecuaciones 2.1 y 2.2 se reduce al sistema: 


$$
\frac{d Y}{d r}=A Y
$$

donde $Y=\left(U_{n}, V_{n}, T_{r r, n}, T_{r \theta, n}, \Phi_{n}, Q_{n}\right)^{t}$. $A$ es una matriz de $6 \times 6$ que depende del modelo de Tierra y del grado del armónico esférico $n$. ver Longman (1962).

Para determinar la respuesta de la Tierra se resuelve la ecuación 2.5 relativo a las condiciones de frontera apropiadas.

\subsubsection{Números de Love}

Los números de Love y Shida adimensionales, $h_{n}(r), I_{n}(r)$ y $k_{n}(r)$, se introdujeron en el caso de una Tierra esférica no rotante con el fin de describir las propiedades elásticas de esta. Estos caracterizan la respuesta de la Tierra a la atracción luni-solar. Posteriormente fueron generalizados para el caso de un potencial de carga externa, donde la única diferencia entre las condiciones de contorno asociadas es que en la superficie libre en el segundo caso ejerce una tensión normal que falta en el primero. Los llamados números de Love de carga se suelen distinguir de la siguiente manera: $h_{n}^{\prime}, l_{n}^{\prime}$ y $k_{n}^{\prime}$ (Farrell, 1972; Francis and Dehant, 1987).

La respuesta de la Tierra a las cargas superficiales se resuelve al integrar la ecuación de movimiento, la relación entre la tensión-deformación y la ecuación de Poisson dentro de la Tierra, desde el centro de masa hasta la superficie. Dichas ecuaciones quedan parametrizadas por las propiedades reológicas de la Tierra, dicho de otro modo, los números de Love de carga dependen de los "modelos sismológicos"(Francis and Dehant, 1987). Los números de Love de carga pueden obtenerse de las soluciones a la ecuación 2.5, dichas soluciones se expresan en términos de los números adimensionales de Love, cada uno de los cuales es función de $n$ y $r$. Cuando las componentes de los desplazamientos $U_{n}, V_{n}$ y $\Phi_{1, n}$ surgen de un campo de fuerzas axialmente simétrico con un potencial $\phi_{2}$ y coeficientes $\Phi_{2, n}$, se definen de la siguiente manera (Farrell, 1972),

$$
\left[\begin{array}{c}
\frac{U_{n}(r)}{g} \\
\frac{V_{n}^{g}(r)}{g} \\
\Phi_{1, n}(r)
\end{array}\right]=\Phi_{2, n}(r)\left[\begin{array}{c}
\frac{h_{n}(r)}{g} \\
\frac{l_{n}(r)}{g} \\
k_{n}(r)
\end{array}\right]
$$

y dado que, nuestro interés esta en los desplazamientos en la superficie, el valor de $r=a$ corresponde al radio medio terrestre.

Love (1911) consideró la propiedad de ortogonalidad de las funciones armónicas por lo que todas las deformaciones causadas por las fuerzas derivadas de dicho potencial perturbador podían representarse, con suficiente aproximación, mediante un desarrollo en serie de armónicos esféricos, siendo cada uno de sus términos proporcional al de igual grado del desarrollo del potencial. Definiendo así los números de Love. 


\subsubsection{Funciones de Green}

Las Funciones de Green se definen como la deformación de la superficie de la Tierra debido a una masa puntual en la superficie de esta. Para obtener dichas funciones es necesario calcular las series infinitas de los polinomios de Legendre ponderados con los números de Love de carga $\left(h_{n}^{\prime}, l_{n}^{\prime}, k_{n}^{\prime}\right)$. Las funciones de Green representan la respuesta de la Tierra en función de la distancia angular entre el punto de observación y el punto de aplicación de la carga. Los dos grupos de funciones de Green que nos interesan son las componentes de los desplazamientos y aceleraciones, dado que son los principales observables de los efectos de carga (Wang et al., 2012; Peltier and Andrews, 1976; Farrell, 1972; Longman, 1962, 1963; Scherneck, 1990).

El desplazamiento de la componente vertical, horizontal, aceleración y potencial, respectivamente, a una distancia $\theta$ del punto en donde se aplica la carga de 1 kilogramo es

$$
\begin{gathered}
u(\theta)=\frac{a}{m_{T}} \sum_{n=0}^{\infty} h_{n}^{\prime} P_{n}(\cos (\theta)), \\
v(\theta)=\frac{a}{m_{T}} \sum_{n=1}^{\infty} I_{n}^{\prime} \frac{\partial P_{n}(\cos (\theta))}{\partial \theta}, \\
g(\theta)=\frac{g_{0}}{m_{T}} \sum_{n=0}^{\infty}\left[n+2 h_{n}^{\prime}-(n+1) k_{n}^{\prime}\right] P_{n}(\cos (\theta)), \\
\phi(\theta)=\frac{a g_{0}}{m_{T}} \sum_{n=0}^{\infty}\left[1+k_{n}^{\prime}\right] P_{n}(\cos (\theta)),
\end{gathered}
$$

determinando así las funciones de Green para el desplazamiento, aceleraciones y potencial en función de la distancia angular $\theta$, donde $m_{T}$ es la masa de la Tierra normalizada con la masa unidad. Tanto para gravedad como potencial, la sumatoria del primer termino entre corchetes es la aceleración y el potencial directo o newtoniano correspondiente a la carga.

Dado que los números de Love presentan un comportamiento asintótico es factible reescribir las expresiones de las funciones de Green mediante la transformación de Kummer, permitiendo así una mejor convergencia de la serie, aunque igualmente lenta, dado que la amplitud de los $P_{n}$ decae a razón de $n^{-1 / 2}$; otras consideraciones se tienen en cuenta para acelerar la convergencia (Farrell, 1972; Francis and Dehant, 1987). 


\subsubsection{Integral de convolución, cálculo de efectos de carga}

$\mathrm{Al}$ convolucionar las funciones de Green para un determinado efecto con la carga superficial se obtiene la respuesta de la Tierra en la superficie. Expresando la convolución espacial de la siguiente manera:

$$
L\left(\varphi^{\prime}, \psi^{\prime}\right)=\int G(\theta) m(\varphi, \psi) d m
$$

donde,

$$
\begin{aligned}
& L\left(\varphi^{\prime}, \psi^{\prime}\right) \\
& (\varphi, \psi) \\
& \left(\varphi^{\prime}, \psi^{\prime}\right) \\
& \theta \\
& G(\theta) \\
& m(\varphi, \psi)
\end{aligned}
$$

Efecto de carga en el punto de observación Coordenadas de la carga Coordenadas del punto de cálculo Distancia esférica Función de Green Distribución de la masa

Las funciones de Green se caracterizan por tener una singularidad de primer orden en el origen. Una manera eficiente de resolver esto es utilizando celdas limitadas por ángulos geocéntricos y azimutales con centro en el punto de cálculo, similar al método de plantilla (Heiskanen and Moritz, 1967), en lugar de usar áreas limitadas por latitud y longitud, de esta manera aparece un seno de la distancia $\theta$ en la integral, esto permite tratar la singularidad (Goad, 1980; Agnew, 1997).

Los modelados desarrollados en este trabajo comprenden los efectos de carga para cuatro funciones de Green, siendo estas, deformación vertical, horizontal, cambio de gravedad y cambio de potencial. Los cálculos han sido han sido desarrollado para todas estas componentes mencionadas, aun encontrándose en proceso de validación las componentes horizontales. Otros efectos de carga pueden ser determinados de estos mismos efectos, como es el cambio de altura ortométrica $H$, el cual requiere del efecto de carga de potencial y deformación vertical para ser determinado, o cambios en la inclinación $t$, que pueden ser derivados del cálculo de gradientes del cambio de altura ortométrica (calculó de gradiente según Horn (1981) mediante herramientas de Wessel et al. (2013)).

$$
\begin{gathered}
H(\theta)=\frac{\phi(\theta)}{g_{0}}+u(\theta), \\
t(\theta)=\|\nabla H(\theta)\| .
\end{gathered}
$$




\subsection{Modelos de carga}

Según la metodología que se ha seguido, los modelos de carga se componen de archivos con forma de cuadrícula rectangular, puntos con valores de las coordenadas del punto y su variación representada en la vertical (longitud, latitud y variación). Esto representa una cuadrícula cuyas celdas están centradas en el punto de coordenadas conocidas, con superficie proporcional a los intervalos en longitud y latitud. Esto permite el cálculo de convolución con las funciones de Green para cada elemento de masa centrada en la celda. Los modelos representan el cambio en la vertical como cambio de masa para un solo paso temporal, el modelo ingresado se proporciona en unidades de metros para obtener la salida en unidades de $[\mathrm{mm}]$, $[\mu \mathrm{gal}] \mathrm{y}\left[\mathrm{m}^{2} / \mathrm{s}^{2} 10^{-3}\right]$, para desplazamientos y/o desplazamientos, gravedad y potencial, respectivamente. Son diversas las cargas que se consideran para el cálculo de efectos. En el capítulo 4 se amplia la información respecto a la construcción de modelos de carga oceánica, carga atmosférica, carga hidrológicas y carga glaciar utilizados.

El cálculo de efectos de carga utilizando las funciones de Green tiene la ventaja no solo de ser más rápido computacionalmente, sino también de alcanzar un mayor grado de resolución espacial, al permitir la división de la carga a geometrías más precisas. Algo muy distinto en comparación con el desarrollo en armónicos esféricos, el cual para obtener mayor resolución espacial requiere el desarrollo de muy alto grado, difícil de alcanzar e implicando mayor tiempo computacional.

\subsection{Modelos de Tierra: modelos SNREI, funciones de Green para G-B y PREM}

La forma idealizada más habitual de representar la Tierra es mediante los modelos de Tierra SNREI (Spherical - NonRotating - Elastic - Isotropic), donde se considera un modelo compuesto por capas esféricas, homogéneas e isótropas, con parámetros elásticos y densidad dependientes del radio. En principio, estos parecen ser válidos para mareas terrestres, las cuales actúan sobre toda la Tierra, más que para efectos de carga oceánica (aplicación más difundida), cuyos efectos son apreciables solo para la Litosfera (Farrell, 1972). Por lo que, diferencias en la estructura de la Tierra, como por ejemplo cuencas oceánicas y continentales, verán afectada en mayor medida los efectos de carga oceánica que de marea terrestre (Farrell, 1972). Esto está basado en la idea que las funciones de Green son sensibles a las estructuras regionales de la corteza y manto dentro de cierto tamaño de escala si la carga esta situada dentro de una distancia de escala semejante; a distancias mayores a $500 \mathrm{~km}$, las deformaciones se comportan como si la Tierra tuviese una estructura lateral homogénea como la obtenida de modelos globales de Tierra. Heterogeneidades someras debajo de la carga, punto de observación y la región entre estos causa un menor impacto en la respuesta a la carga a medida que la distancia entre la carga y el punto aumentan (Scherneck, 1990).

Una parte clave de la teoría de la respuesta dinámica de la Tierra a las fuerzas externas 
o internas proviene del estudio de las oscilaciones libres. Siendo fundamental para la sismología, los modos normales de la Tierra, u oscilaciones libres, son ondas estacionarias a lo largo de la superficie y el radio de la Tierra. El estudio de las oscilaciones libres en sí mismo se ocupa de analizar y extraer información en períodos muy largos (período de 3000-100 s), ya que en este rango de períodos los modos intrínsecos de ondas estacionarias de oscilación son evidentes en los espectros sísmicos. (Woodhouse and Deuss, 2015). Los diferentes modelos de Tierra que se utilizan para describir la estructura interna de la Tierra se basan en observaciones indirectas. Esta información se obtiene a partir de la propagación de ondas sísmicas, las velocidades de propagación dependen de la densidad y del módulo elástico del medio, entre otras. Estudios de la precesión, nutación, mareas terrestres y oscilaciones libres en geodesia y geofísica, requieren de información precisa de las propiedades y estructura interna de la Tierra. La importancia de las nutaciones libres y forzadas, por ejemplo, el movimiento polar, las componentes de Chandler y anuales, y el movimiento diurno de la Tierra, en los diferentes campos de la ciencia, pone de manifiesto el valor de la contribución de la sismología para estas investigaciones (Dziewonski and Anderson, 1981).

Comparaciones en los cálculos de efectos de carga utilizando las funciones de Green con base en los modelos de Tierra más difundidos, Gutenberg-Bullen y PREM (Preliminary reference Earth model; (Dziewonski and Anderson, 1981)), han sido llevadas a cabo por Scherneck (1990). Diferencias con respecto a los resultados utilizando ambas funciones y método provisto por Farrell (1972) tienden a ser pequeñas en la mayoría de las componentes. Cálculos de carga oceánica comparando las distintas funciones de Green concluyen que errores en los modelos regionales y globales de marea oceánica son aproximadamente un orden de magnitud más importante que las funciones de Green de diferentes modelos de tierra solida (Scherneck, 1990).

Los modelos de Tierra seleccionados para el cálculo de efectos de carga quedan representados por las funciones de Green. Dichas funciones intervienen en el cálculo proporcionando la respuesta a una determinada carga. O de manera equivalente, las funciones de Green determinan el comportamiento del modelo a fuerzas externas aplicadas sobre el mismo. Queda claro que los modelos de Tierra mencionados corresponden a modelos estándar que representan valores reológicos medios de la Tierra. Esto corresponde con los resultados obtenidos por Scherneck (1990).

\subsection{ELOADE: una herramienta para el cálculo de efectos elásticos de carga}

Se desarrolló un código en $\mathrm{C}++$ dedicado al cálculo de efectos de carga según Farrell (1972). Program «ELOADE» - Calculation of Elastic LOADing Effects on a sphere - (Cálculo de efectos elásticos de carga sobre una esfera). El programa realiza la integral de convolución entre los elementos del modelo de carga y las funciones de Green para un modelo de Tierra dado. El cálculo se realiza de manera secuencial para los puntos de cálculo que son seleccionados. Como herramienta fundamental para el cálculo y almace- 
nar variables se utilizan punteros. La compilación se realizó con GNU C++ versión 6.3, compilador estándar de código abierto.

Para su uso, siendo este muy simple, el cálculo requiere de tres archivos de entrada en formato ASCII:

- Modelo de tierra: archivo de cinco columnas, estas son, distancia angular y las funciones de Green para las deformaciones superficiales vertical, horizontal, gravedad y potencial, respectivamente. En este trabajo se utilizaron las funciones de Green proporcionadas por Farrell (1972) del modelo Gutenberg-Bullen a excepción de la función de Green para el potencial, correspondiente al modelo PREM (Francis and Mazzega, 1990). Funciones de Green correspondiente a otros modelos de Tierra que se dispongan pueden ser utilizadas por el código.

- Modelo de carga: archivo de tres columnas, consta de longitud, latitud y variación (estando representada por altura en metros de cada elemento de carga). Este representa una cuadrícula regular, donde el equiespaciado para los elementos de carga en longitud y latitud no requieren ser iguales. Sin embargo, las coordenadas deben seguir un orden predeterminado, secuencialmente comenzando en el punto extremo noroeste de la cuadrícula de carga recorriendo hacia el este y luego bajando en latitud hasta terminar en el extremo sureste.

- Archivo de parámetros: «eloade.par», donde consta de varias filas con la siguiente información en orden:

- Densidad de la masa de carga en $\mathrm{kg} / \mathrm{m}^{3}$.

- Ubicación y nombre del archivo con las funciones de Green.

- Cantidad de filas para las funciones de Green.

- Cantidad de columnas para las funciones de Green.

- Ubicación y nombre del archivo de carga.

- Cantidad de puntos en la dirección este-oeste para el archivo de carga.

- Cantidad total de filas del archivo de carga.

- Nombre del modelo de Tierra.

Y por último, las coordenadas de los puntos donde se quieren obtener los efectos indicando longitud y latitud, respectivamente, las cuales se ingresan por standard input (pudiendo ser fácilmente ingresadas mediante un pipe en un script). Es importante mencionar que tanto el modelo de carga como los puntos de cálculo deben ser representados en correspondencia el uno del otro, en el mismo sistema de coordenadas geográficas y grados decimales. Así mismo el modelo de carga debe ser ingresado en unidades de metros para corresponder con las unidades de los efectos calculados.

A continuación se presenta una breve descripción del código y su funcionamiento. El mismo consta de las siguientes funciones:

* ReadData: lectura de archivos de datos que se almacenan en punteros a punteros.

* SphericalDistance: calcula la distancia esférica entre dos puntos. 
* Mass: calcula la masa total de la carga.

* Azimut: determina el azimut desde un punto de cálculo a un elemento de carga.

* SplineCoeff: determina los coeficientes de la interpolación spline cubic en base a las funciones de Green.

* SplineInterpolation: realiza la interpolación spline cubic sobre las funciones de Green para las distancias determinadas por SphericalDistance.

* BilinearInterpolation: realiza la interpolación bilineal para los elementos de carga cercanos (nearby load elements) sobre la cuadrícula del modelo de carga.

* CoordinatesTransformation: transforma azimut y distancia esférica de los elementos cercanos a coordenadas geográficas.

* Convolution: realiza la integral de convolución para cada uno de los efectos, deformación vertical, horizontal, cambio de gravedad y cambio de potencial. El cálculo se divide en puntos lejanos (distant load elements) y cercanos (nearby load elements) a los elementos de carga de manera de tratar la singularidad de las funciones de Green en el origen.

Al iniciar el computo, el algoritmo requiere almacenar en memoria la cuadrícula del modelo de carga completa, junto con las funciones de Green, por lo que el tamaño y resolución de la misma se encuentra limitada a la memoria disponible (cálculos con archivos de carga de tamaño mayor a 1 GB se llevaron a cabo). Como se mencionó, la convolución con los elementos de carga se divide en dos pasos y todo el computo se lleva a cabo de manera secuencial sobre cada punto de cálculo. Partiendo con base en la resolución de la cuadrícula de carga se determina un valor límite (threshold) para dividir los elementos de carga en lejanos y cercanos al punto de cálculo. En el caso de los elementos lejanos, se aplica simplemente la convolución entre el elemento de carga y la función de Green interpolada (SplineInterpolation) para la distancia esférica correspondiente. Un caso distinto se aplica a los elementos cercanos, donde el procedimiento por el que se ha optado es representar a estos como secciones de anillos concéntricos, subdividiendo un disco de área equivalente al de la superficie de todos los elementos cercanos, de forma radial y azimutal con centro en el punto de cálculo. Sobre cada elemento de anillo, con centro de masa según la distancia radial y azimut (las coordenadas geográficas se determinan con la función CoordinatesTransformation) se determina el valor de la carga por interpolación (uso de la función BilinearInterpolation). Una vez determinado el valor de carga por cada sector de anillo, se suman los efectos de cada uno. De esta manera se determinan los efectos de las masas cercanas. Al finalizar se suman los efectos de puntos cercanos y lejanos para todos los elementos de la cuadrícula de carga. Si desea obtener el código ELOADE.cpp y entrar en detalle del procedimiento, dirija un e-mail a la casilla de correo emarderwald@fcaglp.unlp.edu.ar. 


\section{Capítulo 3}

\section{Patagonia austral: sinopsis geofísica-geomorfológica-geográfica}

Nos centramos en la región de Patagonia austral (ver figura 3.1), esta se encuentra comprendida entre los países de Argentina y Chile, donde nuestro interés se concentra comenzando en la latitud correspondiente al límite norte de la provincia de Santa Cruz hasta la Isla Grande de Tierra del Fuego inclusive. Región que exhibe una gran variabilidad geomorfológica y climática debido a su gran extensión y al accionar de procesos geomorfológicos y tectónicos, así como a su situación geográfica. Territorio cuyo potencial hídrico actual se concentra en los Campos de Hielo Patagónico y precipitaciones nivales.

Es en este sector de la Patagonia donde el modelado glaciar alcanzo su máxima extensión areal y temporal. El período cuaternario de la Cordillera Patagónico-Fueguina está caracterizado por el mayor desarrollo relativo, tanto areal como estratigráfico, de los depósitos glaciogénicos en América del Sur. Estos fueron generados por un manto de hielo y glaciares de montaña, extendidos en forma continua desde el paso Pino Hachado en Neuquén (latitud $38^{\circ} 44^{\prime}$ Sur; longitud $70^{\circ} 56^{\prime}$ oeste) hasta el Cabo de Hornos (latitud $55^{\circ} 59^{\prime}$ Sur; longitud $67^{\circ} 17^{\prime}$ oeste). Las enormes dimensiones de este manto de hielo de montaña lo hacen el mayor de cuantos han existido durante el Pleistoceno en el Hemisferio Sur, fuera de la Antártida. Hacia el final del pleistoceno temprano (quizás pre-límite BrunhesMatuyama, 0,73 Ma.), se produjo un importante episodio de levantamiento y/o cambio climático regional, que provocó la génesis y profundización de las líneas de drenaje como las conocemos en la actualidad (Canyon-cutting). Se desarrollaron entonces condiciones de glaciación de montaña con glaciares de descarga encauzados en sistemas de valle. Los lagos se originaron principalmente como resultado de la excavación glaciar pleistocena, la depositación de morenas terminales y el consecuente retroceso (o derretimiento total) de los glaciares de origen. En particular, durante el último máximo glaciar denominado Nahuel Huapi en Argentina (Flint and Fidalgo, 1964) (aproximadamente 20.000 años atrás).

Geológicamente, la Isla Grande de Tierra del Fuego se divide en tres zonas estructurales (de noreste a suroeste): la cuenca de Magallanes, el cinturón de plegamiento y 


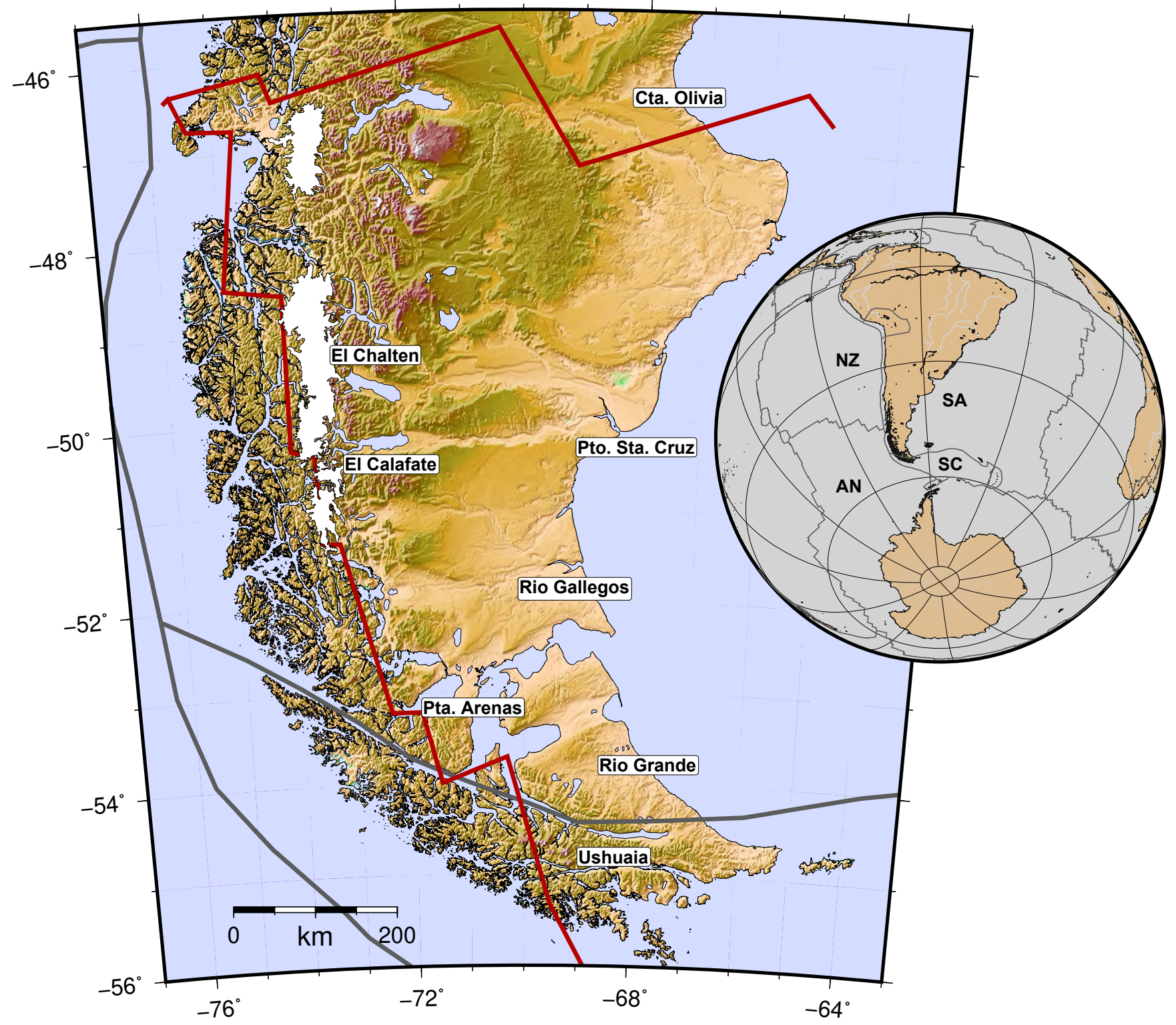

Figura 3.1: Mapa de la región que comprende este trabajo. Se indican limites de placas tectónicas (líneas grises), representadas también en el globo terráqueo (NA: Nazca; SA: Sudamericana; AN: Antártica; SC: Scotia) de acuerdo a Bird (2003). Líneas rojas delimitan la ventana astenosférica de acuerdo a Breitsprecher and Thorkelson (2009). Se incluyen algunas de las principales localidades. Topografía: ETOPO1 modelo de relieve global de 1 minuto de arco (Amante and Eakins, 2009).

empuje de Magallanes y los Andes Fueguinos (Olivero and Martinioni, 2001; Menichetti et al., 2008); este último representa el más meridional segmento de la Cordillera de los Andes. El entorno tectónico actual está controlado principalmente por el sistema de fallas Magallanes-Fagnano (Lodolo et al., 2003). De acuerdo con los resultados geológicos, geomorfológicos y geofísicos, el sistema se caracteriza por un régimen de deslizamiento lateral-izquierdo, el cual representa el límite actual de placas tectónicas Sudamericana- 
Scotia (ver figura 3.1) en esta región y acomoda sus movimientos relativos (Pelayo and Wiens, 1989; Lodolo et al., 2003).

La Patagonia austral actualmente se destaca por tener la acumulación más grande de hielo continental extrapolar en el hemisferio sur (Casassa et al., 2014) y por su gran tasa de cambio en su masa glaciar (Richter et al., 2019; Willis et al., 2012a,b). Estos cambios ocurren cerca del límite tectónicamente activo entre las placas Antártica y Sudamericana. La fuerza mecánica del lado continental está influenciada por la subducción de la placa oceánica joven. Donde una cadena de volcanes jóvenes se presenta paralela a la costa del Pacífico. Una situación tectónica-reológica particular, determinada por la subducción de la dorsal oceánica activa en el Punto Triple de Chile (Bird, 2003; Russo et al., 2010), la apertura de la Ventana Astenosférica Patagónica (Gorring and Kay, 2001; Breitsprecher and Thorkelson, 2009) y el movimiento transformante entre las placas de Sudamérica y Scotia en Tierra del Fuego (Mendoza et al., 2015). Evaluar la influencia de esta estructura sobre la tasa observada de elevación de la tierra viene siendo un tema de enfoque internacional (Ivins and James, 1999, 2004; Klemann et al., 2007; Richter et al., 2016) (ver figura 3.1).

La zona occidental dominada por la orogenia andina es la causa por la cual la Patagonia extra-andina presenta su actual grado de aridez al situarse en sombra orográfica. Muchos de los lagos tienen su orilla oeste cerca de glaciares o hielos continentales, algunos de ellos se alimentan en gran parte de las aguas de deshielo de estos glaciares. Sobre este sector se reciben abundantes precipitaciones. Esto, en consecuencia, propició el desarrollo de densos bosques y vegetación exuberante sobre sus orillas de laderas escarpadas. En esta región los lagos presentan largos y angostos brazos de estructuras intrincadas y muy profundas, brazos similares a fiordos (por ejemplo costa sobre el Pacifico de Patagonia Austral, Noruega, Nueva Zelanda). Estas son artesas, geoformas de erosión glaciar de gran evidencia. A partir del momento en el que los valles fueron abandonados por los glaciares, transitaron por una etapa periglaciar con gran inestabilidad de sus laderas (remoción en masa) logrando recién cierta estabilidad cuando estas pendientes fueron cubiertas por la vegetación. Algunos de dichos valles fueron ocupados por cuerpos lacustres mayoritariamente hacia las pendientes al Este o el mar (Vertiente pacífica), mientras que otros comenzaron a ser modelados por la acción fluvial.

En el este, la Patagonia Extra-andina, a diferencia del ambiente orográfico precedente, está dominada en cambio por un paisaje en el cual la unidad del relieve está representada por superficies subhorizontales, de suave inclinación regional hacia el este y compuestas por mesetas basálticas, extensas terrazas glacifluviales y planicies aluviales. En este ambiente no se aprecian serranías irregulares, más allá de relictos mesetiformes, lomadas morénicas y pequeños aparatos volcánicos. La existencia de geoformas, aun siendo antiguas, es claramente identificable debido a la baja intensidad con la que actúan los procesos fluviales postglaciares, situación que está directamente influenciada por la característica climática que presenta este ambiente. Mientras la región cordillerana posee un clima frío oceánico con precipitaciones importantes sobre la divisoria de aguas continentales (10.000 mm anuales, (Casassa and Rivera, 1999)), hacia el este, en el ambiente correspondiente al sector precordillerano, estos valores decrecen marcadamente alcanzando tan solo 300 $\mathrm{mm}$ anuales; esto implica condiciones climáticas semiáridas. Hacia la región de Patagonia 
Extra-andina las precipitaciones disminuyen aún más, hasta alcanzar valores por debajo de los $200 \mathrm{~mm}$ anuales. En toda la región prevalecen fuertes vientos del oeste. En este ambiente, el bioma es del tipo estepa arbustiva semiárida (estepa patagónica, según Roig (1998)). El sector este de los lagos, en comparación con el oeste, presenta aguas menos profundas y orillas de poca pendiente, de trazado suave y redondeado. Las orillas orientales de los lagos, en muchos casos, están conformados por morenas terminales constituidas por depósitos de till que marcan la extensión de los glaciares durante el último máximo glaciar. El régimen hidrológico de estos lagos y sus cuencas está caracterizado por el hecho que durante una parte determinada e importante del año las precipitaciones caen en forma sólida (nieve) y se acumula durante varios meses en las zonas altas. Como consecuencia, estos lagos experimentan variaciones estacionales del nivel del agua, con un mínimo en invierno (cuando la precipitación se acumula en forma de nieve en las montañas) y un máximo en verano (cuando la lluvia se suma al agua de deshielo de los glaciares).

Sobre la margen atlántica se presentan amplitudes excepcionales de las mareas oceánicas sobre una de las plataformas marinas más extensas en la Tierra (Richter et al., 2012b; Glorioso and Flather, 1997). Región caracteriza por sus condiciones adversas debido a los fuertes vientos y corrientes. Dadas sus dimensiones, con un ancho cercano a la cuarta parte de la longitud de onda de las mareas semidiurnas, la plataforma patagónica favorece la absorción resonante de la energía de las mareas (Webb, 1976). La circulación sobre la plataforma depende de la propagación de las olas de marea, el forzamiento del viento, las descargas de agua dulce y la influencia de las corrientes limítrofes vecinas del oeste. La contribución relativa de cada uno de estos forzamientos a los patrones de circulación varía entre regiones (Saraceno et al., 2010). La anchura media de la plataforma continental sudoccidental alcanza los $800 \mathrm{~km}$ a los $51^{\circ}$ llegando hasta los $55^{\circ}$ de latitud sur y desde la línea de costa hasta la isóbata de $300 \mathrm{~m}$, donde una pronunciada ruptura de la plataforma divide claramente las aguas continentales de las aguas abiertas.(Saraceno et al., 2010). Los modelos de Glorioso and Flather (1997) han revelado fuertes interacciones entre las mareas diurnas y las ondas de la plataforma. Las variaciones del nivel del mar sirven en este contexto como componente e indicador observable de estos procesos hidrodinámicos. La descripción del régimen de mareas en la plataforma del Atlántico Sudoccidental se basa principalmente en el análisis de modelos y altimetría satelital. Existen pocos datos in-situ obtenidos a partir de mediciones de corriente y mareógrafos. La plataforma continental del Atlántico sudoccidental presenta un complejo patrón de amplitudes y fases de mareas. El rango de amplitudes de mareas disminuye desde 4,5 $\mathrm{m}$ en el extremo sur de la plataforma Argentina hasta unos pocos centímetros en el sector brasileño. En la región de Patagonia austral la componente mareomotriz que presenta la mayor amplitud en todo el dominio es el semidiurno lunar M2 (hasta 3,7 $\mathrm{m}$ ).

Determinar propiedades del clima de la Patagonia es de extrema dificultad dada la baja disponibilidad de datos. La densidad de las estaciones meteorológicas oficiales es extremadamente baja y se concentran principalmente en las zonas costeras. Aun así, podemos determinar características muy distintivas y marcadas en las distintas zonas. La mayor parte de la Patagonia está dominada por masas de aire procedentes del Océano Pacífico. La región patagónica se encuentra entre los anticiclones semipermanentes de los océanos Pacífico y Atlántico y el cinturón subpolar de baja presión (Paruelo et al., 1998). El clima está caracterizado por una componente dominante, el fuerte viento que se 
observa en casi toda región, característica ampliamente observada en la Patagonia y su ocurrencia con una relativamente alta frecuencia. Este es un factor adicional de aridez ya que favorece la evaporación en un contexto de escasas precipitaciones. Asimismo, es un fuerte factor erosivo que actúa vigorosamente ante las alteraciones de la frágil cubierta vegetal. Los fuertes y constantes vientos del oeste son dominantes en toda la región. El movimiento estacional de los sistemas de baja y alta presión y las corrientes oceánicas hacia el ecuador determinan el patrón de precipitación. Durante el invierno, la baja subpolar es más intensa. Esta situación, combinada con el desplazamiento ecuatorial del alto del Pacífico y con las temperaturas oceánicas que son más altas que las continentales, provoca un aumento de las precipitaciones durante esta estación.

Una característica del clima patagónico es el predominio de los vientos del oeste que se caracterizan no sólo por su persistencia durante el año sino también por su intensidad. Los valores medios anuales de la velocidad del viento variaron entre 15 y $22 \mathrm{~km} / \mathrm{h}$ en la parte centro-oeste de la región. La distribución anual de la velocidad del viento muestra un máximo entre septiembre y enero y un mínimo en invierno. La frecuencia de las calmas fue mayor durante el invierno y puede explicar para hasta el $25 \%$ de las observaciones bihorarias. Los eventos de calma rara vez ocurrieron durante la primavera y el verano (Beltrán, 1997). En el centro-oeste de la región, los vientos del oeste representan entre el 65 y el $75 \%$ de las observaciones diarias del año. Debido al desplazamiento estacional de los sistemas de presión, el invierno tiene una distribución más uniforme de los vientos del oeste, mientras que en el verano es evidente un componente del sur (Beltrán, 1997). Las direcciones predominantes son del oeste, sudoeste y noroeste que en conjunto suman en general alrededor más del $60 \%$ del tiempo. Actualmente, en la provincia de Santa Cruz se presentan los vientos medios anuales más fuertes de la Argentina $(10 \mathrm{~m} / \mathrm{s})$ en la latitud de $48^{\circ}$ Sur. Desde la franja de máximo viento medio anual en el centro de la provincia, este decrece hacia el Sur y hacia el Norte. La zona de máximo viento se desplaza en latitud, estando en $46^{\circ}$ Sur en invierno y entre esa latitud y $49^{\circ} \mathrm{S}$ en verano.

Una característica del patrón de temperatura es la distribución NW-SE de las isotermas, determinada principalmente por la presencia de los Andes. Las temperaturas medias en la región son relativamente frías, debido al efecto de las corrientes marinas en las temperaturas estivales y a la altitud en la meseta y la zona cordillerana. La costa norte de la Patagonia Austral presenta medias de 8 a $9^{\circ} \mathrm{C}$, uno o dos grados más altas que en la meseta, mientras que en el sur las temperaturas medias anuales más bajas se encuentran en los ambientes costeros con valores de $6^{\circ} \mathrm{C}$. Valores inferiores a $5^{\circ} \mathrm{C}$ y hasta debajo de $0^{\circ} \mathrm{C}$ deben esperarse en las zonas altas de la cordillera de acuerdo con el gradiente vertical de temperatura propio de la latitud y las alturas de la cordillera. Durante el verano el gradiente meridional aumenta considerablemente. En efecto, durante enero las temperaturas medias mensuales en el norte se hallan en $\operatorname{los} 16^{\circ} \mathrm{C}$, mientras que en sur se hallan en torno de $\operatorname{los} 12^{\circ} \mathrm{C}$. Las temperaturas máximas extremas han llegado a estar por encima de $35^{\circ} \mathrm{C}$, con excepción de la zona cordillerana. En invierno, el gradiente meridional se reduce notablemente. En julio, las temperaturas medias van de $4^{\circ} \mathrm{C}$ en el norte hasta $2^{\circ} \mathrm{C}$ en el sur. Las temperaturas mínimas extremas se dan en la meseta patagónica y están debajo de los $-20^{\circ} \mathrm{C}$. Los valores mínimos extremos en la región costera son mucho más altos, esto es, mayores a $-15^{\circ} \mathrm{C}$. En la zona costera y sur de Santa Cruz las temperaturas medias del otoño son mayores que las de la primavera, de acuerdo con lo que es típico de 
los climas marítimos.(Paruelo et al., 1998).

La humedad relativa media anual es en general próxima a $60 \%$ y sin grandes contrastes espaciales, con excepción de los ambientes costeros, donde puede superar el $75 \%$. En todos los ambientes sin excepción, la humedad relativa es menor en verano por las mayores temperaturas. En general, la amplitud anual es de un $25 \%$ en humedad relativa. Las precipitaciones nivales son comunes en invierno en toda la región. En el Norte predominan las precipitaciones invernales sobre las estivales. Desde el centro de Santa Cruz hacia el Sur, las precipitaciones son más regulares a lo largo del año, aunque en algunas localidades del extremo Sur existe cierta predominancia de las lluvias de verano como consecuencia de la mayor baroclinicidad que se produce en esa época del año (Paruelo et al., 1998). 


\section{Capítulo 4}

\section{Las Cargas}

El capítulo presenta la descripción de las cargas consideradas en este trabajo, la carga oceánica, atmosférica, hidrológica y glaciar. Su discretización en los modelos de carga, puntos esenciales para su construcción, como tratamientos previos sobre los modelos digitales de terreno necesarios en la determinación de las geometrías de los embalses y masa total. Para cargas que corresponden a eventos cíclicos, como la carga oceánica, se explica el formato para ingresar las componentes armónicas a ser convolucionadas con las funciones de Green.

\subsection{Carga oceánica}

Se conoce con el nombre de efecto de carga oceánica a la respuesta de la Tierra a las fuerzas superficiales ejercidas por la presión de las mareas oceánicas sobre la corteza. Este puede observarse periódicamente en forma de deformaciones de la corteza terrestre en las direcciones vertical y horizontal, como inclinaciones y extensiones, variaciones de gravedad y desplazamientos de puntos observación. Así, los registros de gravímetros, clinómetros y extensómetros utilizados para detectar variaciones de gravedad, desviaciones de la vertical y deformaciones de la superficie terrestre, respectivamente, están directamente afectados. Tanto la marea terrestre como el efecto de carga oceánica tienen el mismo origen astronómico, el potencial luni-solar, por lo que ambos fenómenos tienen el mismo espectro de frecuencias y, como consecuencia, un carácter temporal muy similar. Al contrario de lo que ocurre con su distribución espacial, esta difiere significativamente. En la dirección de la vertical física, estos desplazamientos pueden alcanzar un rango de varios centímetros; para el constituyente armónico de marea M2, la amplitud de la componente vertical del desplazamiento debido al efecto de carga oceánica, puede superar los $5 \mathrm{~cm}$ en algunas zonas costeras (Baker, 1984). La componente horizontal del desplazamiento, es del orden de un tercio de la componente vertical (Farrell, 1972). Por otra parte, en zonas próximas a la costa e islas oceánicas, las variaciones de la gravedad causadas por el efecto de carga oceánica son, en general, del orden de 0,3-3 $\mu$ Gal (Baker and Bos, 2003). Así, las mareas terrestres varían gradualmente sobre la superficie de la Tierra dependiendo, 
principalmente, de las propiedades globales de su reología más que de sus propiedades locales. Sin embargo, la estructura de la variación espacial de la respuesta de la Tierra a las fuerzas ejercidas por la presión de las mareas oceánicas es más compleja. Asimismo, debido a la variación en amplitud de la marea oceánica causada por la propia dinámica del océano, se producen a su vez alteraciones significativas en el efecto de carga oceánica, principalmente en zonas costeras y sus proximidades.

Las medidas de marea terrestre gravimétrica, clinométrica y extensométrica se encuentran afectadas significativamente por el ECO. En zonas próximas a la costa, este efecto es responsable de, aproximadamente, el $10 \%$, el $90 \%$ y el $25 \%$ de las señales de marea registradas en gravedad, inclinación y extensión, respectivamente (Farrell, 1972). El cálculo preciso del ECO puede servir tanto para corregir estas observaciones de su influencia, como para comparar la marea terrestre teórica con la señal observada directamente. Entre otras aplicaciones, la corrección del ECO tiene gran importancia en las medidas de marea terrestre de alta precisión para la predicción de los movimientos de marea y para la determinación de la estructura elástica de la Tierra.

Dado que el mayor efecto de carga oceánica, alrededor de la mitad de este, resulta de la marea en las cercanías con un radio de $2000 \mathrm{~km}$ (Scherneck, 1991), para el cálculo preciso de los efectos de carga oceánica de marea, los modelos de marea oceánica deben proporcionar una buena cobertura global con una buena resolución espacial y con la mayor precisión posible. Mientras que en alta mar se ha logrado una buena precisión en la medición de la superficie del mar medida por diferentes misiones satelitales (TOPEX/Poseidon, Jason-1, Jason-2 y Jason-3). Esto ha permitido lograr una muy buena calidad de los modelos globales de marea oceánica, entre los que se pueden destacar el EOT11a y el FES2014 (Savcenko et al., 2012; Carrere et al., 2016). Ambos modelos se basan principalmente en los datos de altimetría satelital. Sin embargo, en algunas regiones cercanas a la costa y de plataforma continental se presentan problemas de calibración de las misiones altimetrícas satelitales. Dado que las mareas en la zona del atlántico sudoccidental se encuentran entre las más altas del mundo, alcanzando hasta los 12 metros en ciertos lugares específicos (Saraceno et al., 2010), es evidente la necesidad de aplicar correcciones locales a los modelos globales de marea. Por lo tanto, considerando que sobre las regiones costeras el valor de amplitud y fase pueden ser muy diferentes de aquellas predichas por modelos globales (Saraceno et al., 2010; Clarke and Battisti, 1981), modelos regionales de marea deben ser utilizados para complementar y mejorar la información espacial permitiendo una mejora en la precisión.

El régimen de marea en la plataforma oceánica del sector sudoccidental de la Patagonia se encuentra dominado por la componente de marea semidiurna lunar M2 extendiéndose desde la latitud $41^{\circ} \mathrm{S}$ hacia el sur (Glorioso and Flather, 1997; Saraceno et al., 2010). Otras componentes astronómicas de marea constituyen también un gran aporte, como por ejemplo S2, N2, K1 y O1 (Saraceno et al., 2010). Otra característica de esta zona es la significativa intensidad de aguas poco profundas M4, MN4 y MS4 (Glorioso and Flather, 1997; Richter et al., 2012b). En este trabajo se calcularon los efectos de carga para los modelos de marea oceánica EOT11a (resolución de 0,125 grados decimales) y para el modelo regional elaborado por D'onofrio et al. (2016), utilizando las principales ocho componentes de marea M2, S2, N2, K2, K1, O1, P1 y Q1, calculados así mismo por 
el Ocean Tide Loading Provider (Bos and Scherneck, 2011) y utilizados en este trabajo para la validación del código desarrollado.

Una manera de calcular un modelo de carga a ser convolucionado con las funciones de green de carga superficial, es utilizar las constantes armónicas provistas por los modelos de mareas oceánicos globales como EOT o FES y recurrir a modelos locales para la cercanía a la costa. De las distintas componentes dadas en amplitud y fase es posible obtener una cuadricula de carga donde se calculan los máximos efectos de carga oceánica sobre la región continental. Dichas componentes deben estar representadas como componentes de números complejos, real e imaginario, permitiendo así la convolución con las funciones de Green. Para el sector sur correspondiente a Tierra del fuego se utilizó el modelo desarrollado por D'onofrio et al. (2016). Estos autores presentan un modelo regional comprendido entre las latitudes $52^{\circ} 00^{\prime} 00$ " S y $55^{\circ} 20^{\prime} 30^{\prime \prime} \mathrm{S}$ y las longitudes $63^{\circ} 30^{\prime} 00^{\prime \prime} \mathrm{O}$ y $69^{\circ} 00^{\prime} 00^{\prime \prime} \mathrm{O}$, con una resolución de 500 metros, que resulta de combinar datos de mareógrafos costeros con altimetría satelital. Gracias a la incorporación de observaciones mareográficas locales logran mejorar la precisión de la predicción de la señal de marea en aguas costeras. En virtud de esto y a fin de evitar perdida de la resolución espacial, se armó un modelo combinado, formado por tres partes, un modelo global de mareas (EOT11a) recortado en el sector del modelo local, el modelo local de mayor resolución y un recuadro interpolado del modelo global para el sector comprendido entre ambos. Debiendo de esta manera calcular los efectos de manera separada para ser integrada a posteriori.

\subsection{Carga atmosférica}

En la región de Patagonia la serie de datos de las observaciones de presión atmosférica mayormente presentan máximos en el espectro para las componentes diurna, semidiurna, anual y semianual (ver gráfico 4.1). Por otro lado, modelos globales en general son obtenidos por predicciones numéricas (NWP - Numerical Weather Prediction) y ajustados mediante sensores remotos; ajustes por observaciones in-situ se encuentran en gran medida disponible para el hemisferio norte las cuales mejoran los modelos a un nivel regional.

Desde hace tiempo se conocen las deformaciones de la superficie terrestre debido a la variación de la presión atmosférica (Darwin, 1882). Las técnicas geodésicas espaciales pueden detectar deformaciones elásticas de la Tierra causada por la presión de carga atmosférica. Sin embargo, aún no se ha demostrado si estos desplazamientos de la superficie deban incorporados al momento de la reducción de las observaciones o aplicando correcciones sobre las coordenadas a posteriori con valores medios temporales de desplazamiento (Tregoning and van Dam, 2005).

Las componentes de mayor frecuencia de la señal de carga atmosférica pueden propagarse en períodos anuales y semianuales (Penna and Stewart, 2003; Stewart et al., 2005), valores promedio pueden no representar adecuadamente el movimiento real del sitio y, como resultado, la aproximación promedio diaria podría ser inferior a la aplicación de correcciones a nivel de observación. En el caso de la deformación no-mareal, la mejor opción resulta de aplicar carga atmosférica no-mareal a nivel de observación (Tregoning and van 


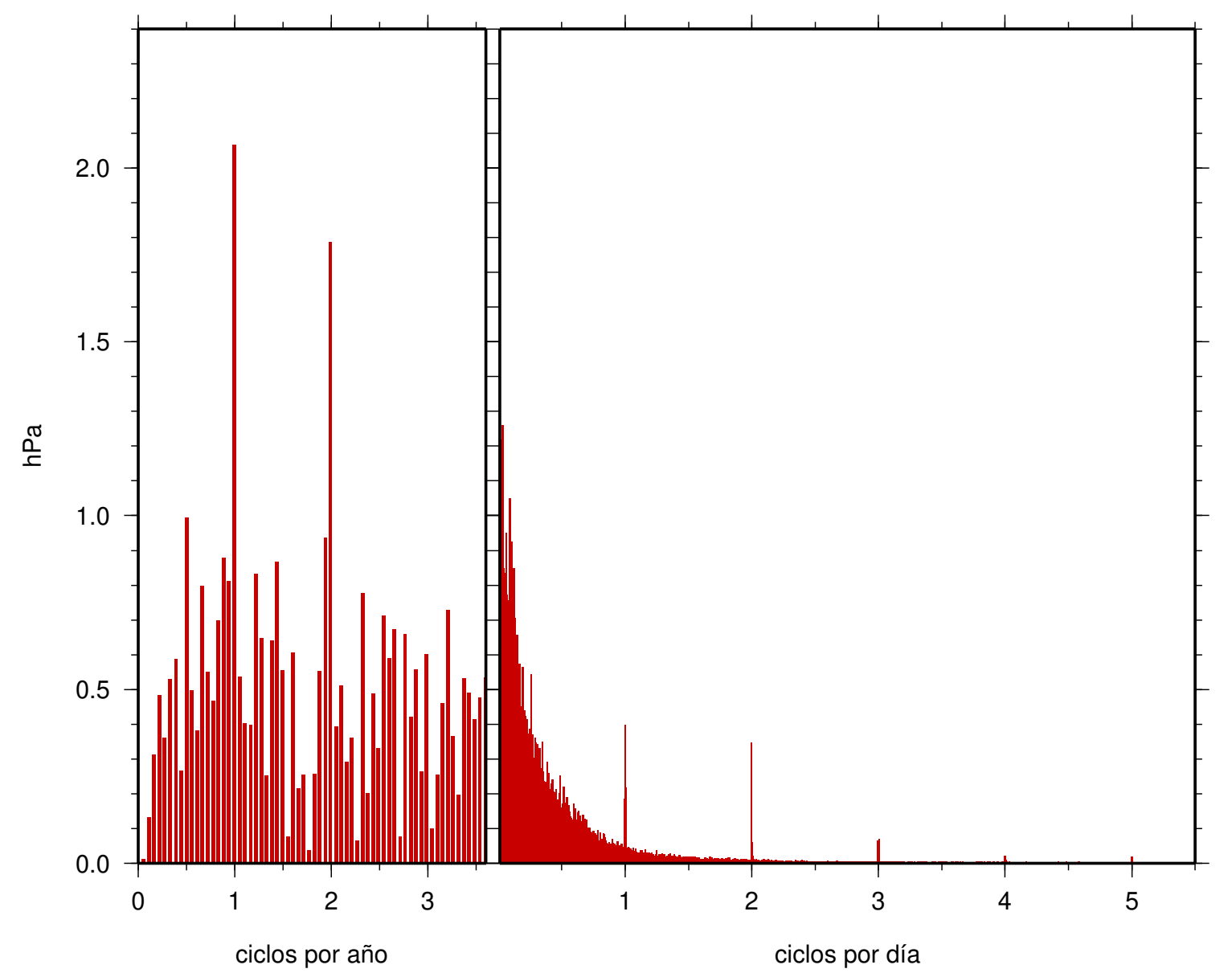

Figura 4.1: Espectro de amplitudes de variación de presión atmosférica observada en la estación sinóptica de Río Gallegos. Se puede distinguir claramente las componentes anual, semianual, diaria y semidiaria.

Dam, 2005).

Con la idea de obtener una estimación de los efectos de carga atmosférica, partiendo de datos sinópticos de presión atmosférica para estaciones meteorológicas de Argentina y Chile (cortesía del Servicio Meteorológico Nacional) se creó un modelo de presión atmosférica regionalizado sobre el continente para las variaciones de las componentes diurna, semidiurna, anual y semianual, estas son S1, S2, SA y SSA, respectivamente, donde se observan los máximos de la densidad espectral. Las variaciones de presión atmosféricas están dominados por un ciclo diurno y un ciclo anual. Ambos no son armónicos. Por este motivo se incluyen las componentes semidiurna y semianual, para describir con mayor precisión los ciclos diurno y anual. Cabe aclarar que estas componentes no representan una fuerza actuando con estos periodos. También, se incluyó un evento particular que tuvo lugar el día 26 de octubre de 2009, donde se muestra con un intervalo temporal que corresponde a las 17, 19 y 22 horas UTC (ver figura 5.6). Estos modelos se obtuvieron de la interpolación de estaciones ubicadas según se muestra en el mapa (ver figura 4.2), derivando en una cuadricula interpolada con una resolución de 0,1 grados longitud y 0,065 grados latitud. Dado que varias de las estaciones no cuentan con suficiente resolución 
temporal, solo algunas de ellas permitieron obtener modelos de carga diaria y semi-diaria (ver figura 4.2).

No se han incluido la respuesta del océano a la presión de carga, a priori una respuesta del barómetro inverso podría esperarse. Pero es necesario partir de una serie de datos observados en tiempo y frecuencia más precisos. Siendo suficiente para obtener valores estimados, donde las variaciones de presión atmosférica suelen ser de muy pequeña magnitud, cuya razón posible podría ser la cercanía al océano en las distintas direcciones y su fácil compensación.

Nos reservamos el uso de la noción de la carga de presión atmosférica únicamente para la deformación de la corteza causada por los cambios de presión atmosférica sobre el continente. Variaciones de la presión atmosférica cambian el nivel del mar. En grandes escalas de tiempo, meses, el cambio del nivel del mar compensa el cambio de la presión atmosférica de manera que la presión del fondo oceánico permanece invariante. El océano responde como el barómetro inverso: a mayor presión, menor el nivel del mar. Cambios en la presión atmosférica causa la redistribución de las masas de agua del océano. El océano responde al barómetro inverso lentamente, que corresponde a cambios de presión atmosférica a escala de un mes o más. A menores escalas temporales la respuesta del océano se desvía ampliamente del modelo de barómetro inverso, por lo que se necesita considerar toda la complejidad de la dinámica oceánica (DO). Modelando la respuesta dinámica del océano es factible con los modelos oceánicos numéricos que toman en cuenta las fuerzas atmosféricas entre otros factores. Las deformaciones de la corteza causadas por cambios de presión del fondo oceánico se denomina carga oceánica no-mareal. Tiene en cuenta los cambios de la presión atmosférica sobre el océano y la respuesta DO a esto, así como el océano repele a otros forzamientos, excluyendo las mareas lunares y solares.

La atracción newtoniana de las masas atmosféricas afecta las mediciones de gravímetros precisas. Para hacer visibles otras señales en series temporales gravimétricas enmascaradas por estos efectos, se debe aplicar una corrección. Hasta el $95 \%$ de los efectos atmosféricos en las series temporales gravimétricas pueden ser eliminados mediante una simple regresión lineal a las variaciones de la presión atmosférica. Sin embargo, esto no es suficiente para muchas aplicaciones en las que se deben interpretar señales pequeñas. Por lo tanto, se debe considerar la distribución espacial de las masas de aire, que se puede realizar utilizando la densidad derivada de los parámetros de los modelos atmosféricos. Para estos fines, Atmacs (BKG - Bundesamt für Kartographie und Geodäsie ) es un servicio para proporcionar series temporales de atracción de masas verticales atmosféricas calculadas sobre la base de los modelos meteorológicos del Servicio Meteorológico Alemán (DWD - Deutscher Wetterdienst), modelo de predicción meteorológica numérica global ICON (Icosaédrico No Hidrostático) (Klügel and Wziontek, 2009). 


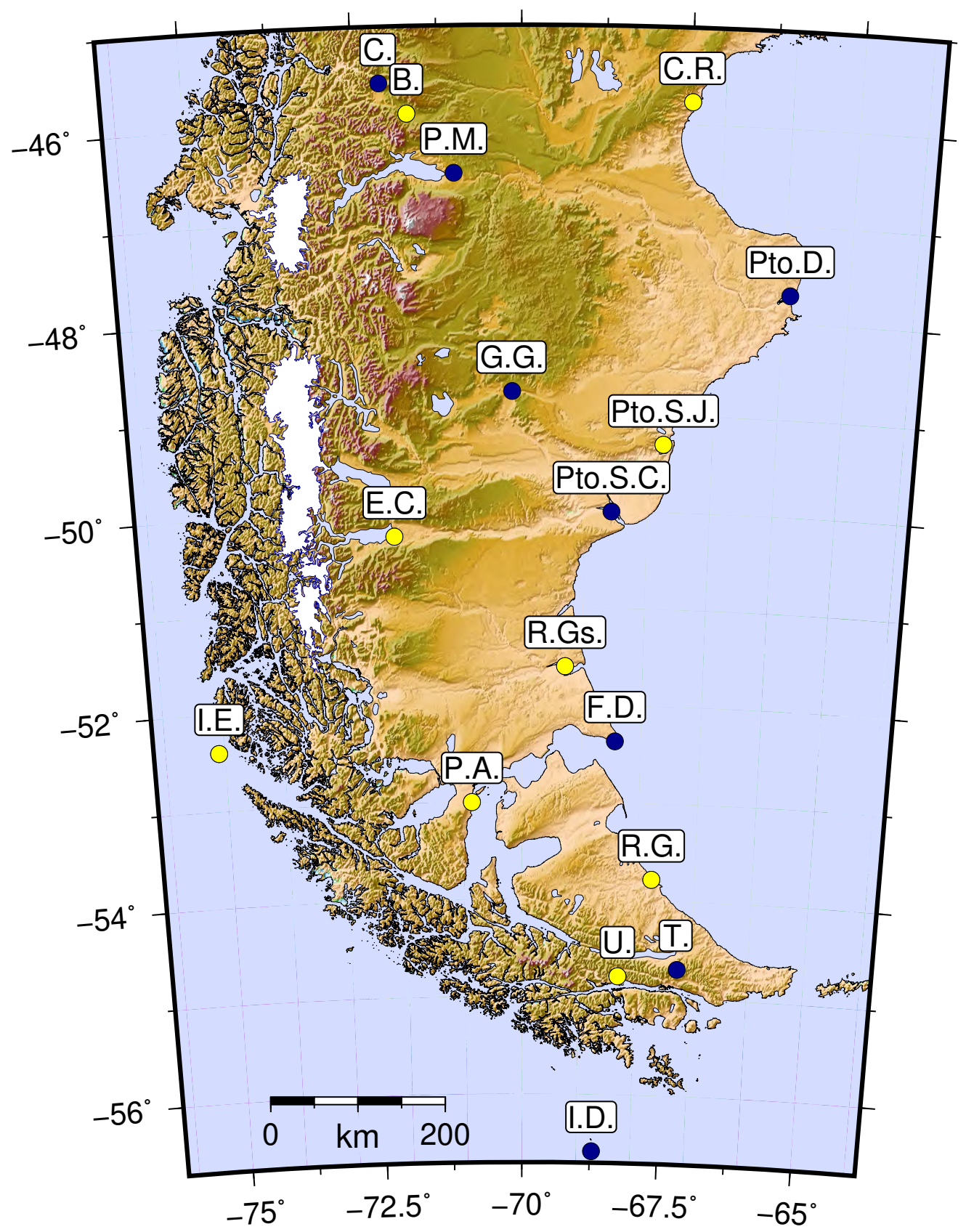

Figura 4.2: Ubicación de las estaciones sinópticas que se dispusieron y analizaron para construir el modelo de carga atmosférica. C.: Coyhaique; B.: Balmaceda; P.M.: Perito Moreno; C.R.: Comodoro Rivadavia; G.G.: Gobernador Gregores; Pto.D.: Puerto Deseado; E.C.: El Calafate; Pto.S.J.: Puerto San Julian; Pto.S.C.: Puerto Santa Cruz; R.Gs.: Río Gallegos; I.E.: Islotes Evangelistas; F.D.: Faro Dungenes; P.A.: Punta Arenas; R.G.: Río Grande; T.: Tolhuin; U: Usuhaia; I.D.: Isla Diego. Solo las estaciones marcadas en amarillo cuenta con la resolución temporal suficiente para obtener modelos diarios. 


\subsection{Carga hidrológica: hidrología continental}

\subsubsection{Los grandes lagos patagónicos}

El sector cordillerano de la Patagonia está caracterizado por la presencia de lagos con características semejantes. El eje principal de estos lagos posee una orientación cercana al eje este-oeste y se extienden sobre la zona de transición entre la ladera oriental de la cordillera patagónica austral en el sector oeste, y sobre el sector Este de la cordillera. La ubicación y orientación de estos lagos está determinada por los valles dispuestos transversalmente a la cordillera, los cuales les une una historia de formación y evolución común. Siendo estos de origen glaciar, reciben su mayor aporte a sus aguas por deshielos del Campo de Hielo Patagónico, dos de estos lagos, lago Argentino y lago Viedma drenan sus aguas hacia el océano Atlántico mientras que otros de los grandes lagos como Buenos Aires/General Carrera, San Matin/O'Higgins y Fagnano lo hacen sobre el océano Pacífico.

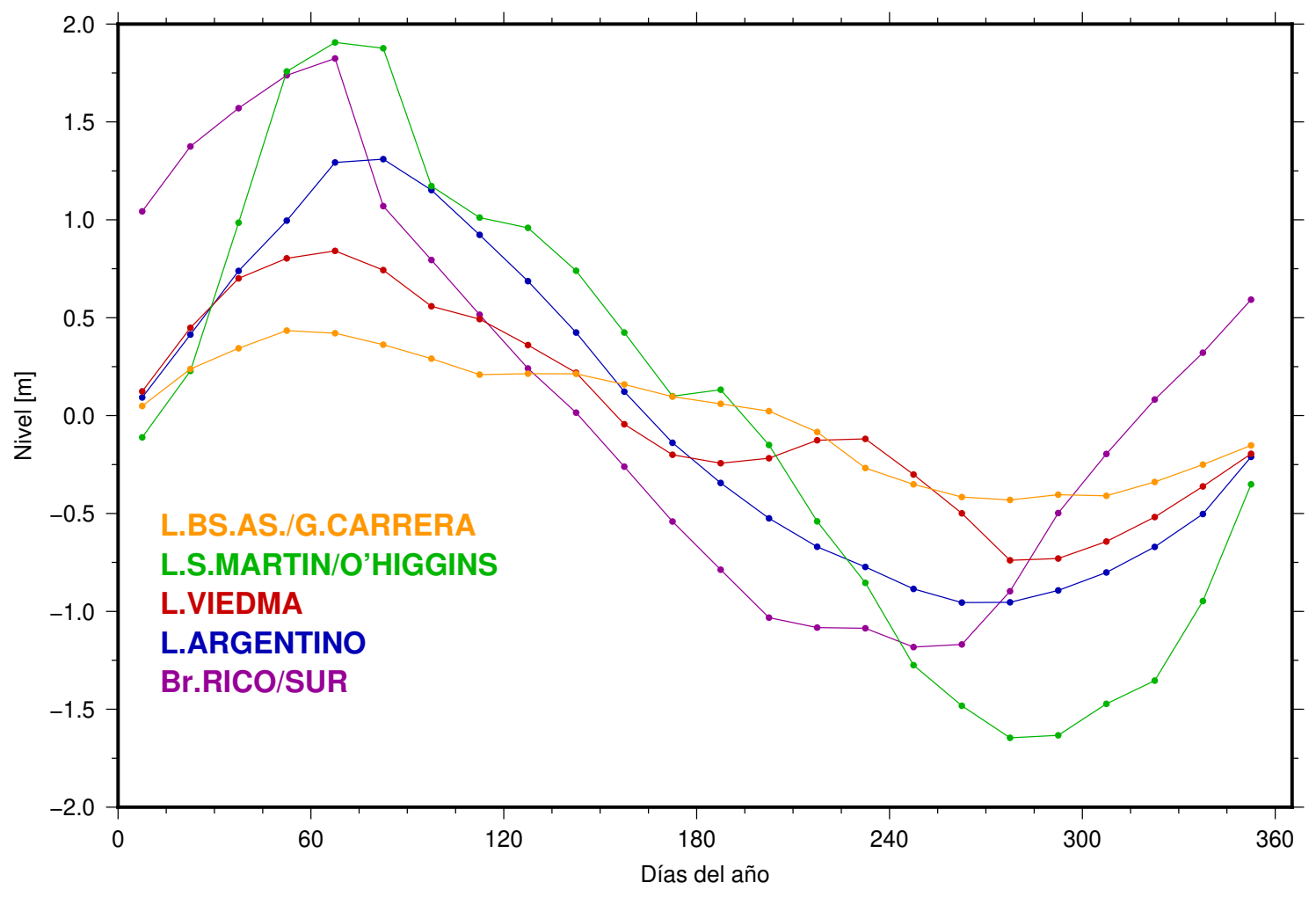

Figura 4.3: Variación anual del nivel de los grandes lagos obtenida través de un apilado con una resolución de 15 días.

Richter et al. (2016) muestra que la variación del nivel del agua en los grandes lagos patagónicos está dominada por un ciclo anual. Estimamos la magnitud de los efectos de la carga hidrológica que representan las variaciones estacionales de la masa de agua en los lagos: Buenos Aires (General Carrera), San Martín (O'Higgins), Viedma y Argentino (considerando a los Brazos Sur y Rico como un lago individual). Sobre la base de los datos de nivel de agua de la Base de Datos de Hidrografía Integrada (BDHI, 2017) se 
determina para cada uno de estos lagos individualmente la señal anual media a través de un apilado con una resolución de 15 días (figura 4.3). La mayor diferencia en la masa total de agua contenida en los lagos (tomando en cuenta el área superficial de cada lago) se produce entre los intervalos correspondientes a principios de marzo (máximo) y principios de octubre (mínimo). Entre estos dos intervalos temporales los desniveles en el nivel de agua en cada uno de los lagos se pueden observar en el siguiente gráfico y se resume en la tabla 4.1. Utilizando estas alturas de agua y las cuadriculas de los lagos (Marderwald, 2014), con una resolución de 5 segundos en longitud y 3 segundos en latitud, se generan modelos de carga que se introducen al cálculo de los efectos de carga elástico.

\subsubsection{Brazos Rico y Sur}

Sobre la margen suroeste del lago Argentino se encuentra el glaciar Perito Moreno, uno de los mayores glaciares en el campo de hielo patagónico sur. A diferencia de la mayoría de los glaciares de la Patagonia, este se encuentra en avance o se ha mantenido estable durante el siglo veinte hasta nuestros días (Guerrido et al., 2014). Los periódicos avances de este glaciar contra la península Magallanes bloquean el canal de los témpanos, ubicado sobre la margen norte del frente glaciar, creando así un endicamiento natural de los brazos Rico y Sur del lago Argentino, ubicados sobre su margen sur, permitiendo de esta manera el aumento del nivel del agua en los Brazos. Los arroyos y ríos afluentes siguen nutriendo los Brazos Rico y Sur, causando de manera progresiva la elevación del nivel de sus aguas. El aumento unilateral de la masa de agua genera gradientes importantes de presión en la base del dique glaciar. Esta fuerza creciente debilita el hielo permitiendo la percolación por debajo de la lengua glaciar con un incremento paulatino del flujo de agua. Este proceso culmina en el rompimiento del frente del glaciar y en consecuencia en el vaciamiento del embalse natural a través del canal de los témpanos, hasta equiparar el nivel con el lago Argentino. Dicho fenómeno se lleva a cabo en un periodo muy corto de tiempo, unos pocos días para el vaciamiento. Estimaciones del caudal promedio han sido realizadas por (Richter et al., 2016) con un valor de $3600 \mathrm{~m}^{3} / \mathrm{s}$ con un nivel de agua en los Brazos Rico y Sur por encima de los 5,5 metros sobre el lago Argentino para el rompimiento ocurrido en marzo de 2012.

Hemos hecho uso de este experimento natural, utilizando dicho endicamiento y se-

Tabla 4.1: Diferencia entre el máximo y el mínimo de la variación anual de cada una de los lagos y su equivalente en masa.

\begin{tabular}{lcc}
\hline Lago & Variación nivel $[\mathrm{m}]$ & Masa $[\mathrm{Gt}]$ \\
\hline BS. AS./Gral. Carrera & 0,85 & 1,5 \\
S. Martin/O'Higgins & 3,55 & 3,6 \\
Viedma & 1,58 & 1,9 \\
Argentino & 2,24 & 2,9 \\
Brazo Rico-Sur & 2,72 & 0,3 \\
\hline
\end{tabular}


Tabla 4.2: Niveles de agua determinados para la construcción de los modelos de carga a ser introducidos en el cálculo de efectos de carga en los Brazos Rico y Sur.

\begin{tabular}{cccl}
\hline Evento & Nivel & Altura ortométrica $[\mathrm{m}]$ & Fuente \\
\hline \multirow{2}{*}{2016} & inicial & 185,876 & BDHI $(2017)$ \\
& máximo & 187,656 & Comisso $(2017)$ \\
& mínimo & 180,616 & Comisso $(2017)$ \\
\hline \multirow{2}{*}{2018} & máximo & 191,716 & BDHI $(2017)$ \\
& mínimo & 181,916 & BDHI $(2017)$ \\
\hline \multirow{2}{*}{1956} & máximo & 204,728 & Guerrido et al. (2014) \\
& mínimo & 180,203 & BDHI $(2017)$ \\
\hline
\end{tabular}

guido del vaciamiento de los Brazos Rico y Sur, cuantificando la señal de carga y su efecto con el objetivo de validar modelos de tierra elástico. (Marderwald et al., 2018a). Utilizando un modelo digital de elevación obtenido por TanDEM-X (TerraSAR-X addon for Digital Elevation Measurement, cortesía de Philipp Malz, Institute of Geography, Friedrich-Alexander-Universität Erlangen-Nuremberg, Alemania), con una resolución espacial de 10 metros. Se desarrollaron diferentes modelos de carga correspondientes a tres episodios de inundación de los Brazos. Estos corresponden a los rompimientos del frente del glaciar Perito Moreno en los años 2016, 2018 y 1956, con alturas del nivel de agua de 7,04, 9,8 y 24,5 metros (ver tabla 4.2), respectivamente (este ultimo tomado como un valor medio entre los mencionados por Mercer (1967); Stuefer (1999); Liss (1970); Heinsheimer (1959). Los niveles máximos y mínimos para cada evento fueron determinaron en base a datos mareógraficos de la Base de Datos Hidrológica Integrada BDHI (2017) y Parques Nacionales (Comisso, 2017) sobre el elipsoide de referencia WGS84. El nivel base del modelo digital de elevación, dado en alturas ortométricas pudo ser ajustado mediante observaciones obtenidas en un relevamiento GNSS cinemático sobre la margen Este del lago en el Brazo Sur, llevado a cabo durante una campaña geodésica en febrero de 2017 (cercanías del punto 3; ver figuras 5.1 y 5.12). Por otro lado, la compensación sobre el lago Argentino al momento del vaciamiento fue tenida en cuenta mediante conservación de la masa.

Se evalúo el uso de otros modelos digitales de terreno para la base del modelo de carga. Un modelo inicial utilizado para obtener modelos de carga se baso en un modelo digital de elevación de ALOS-PALSAR (Advanced Land Observing Satellite - Phased Array type Lband Synthetic Aperture Radar) perteneciente a la Agencia de Exploración Aeroespacial de Japón - JAXA (ALOS-PALSAR, 2017), con una resolución espacial de 12,5 metros, de superior calidad frente a los 30 metros de SRTM (Shuttle Radar Topography Mission, desde la pagina del USGS, (USGS, 2016)) en sus versiones libres. El primero de estos presenta una perdida de precisión en la componente vertical debido que las alturas son dadas cada un metro en resolución vertical. El segundo fue descartado en busca de una mayor resolución espacial. 

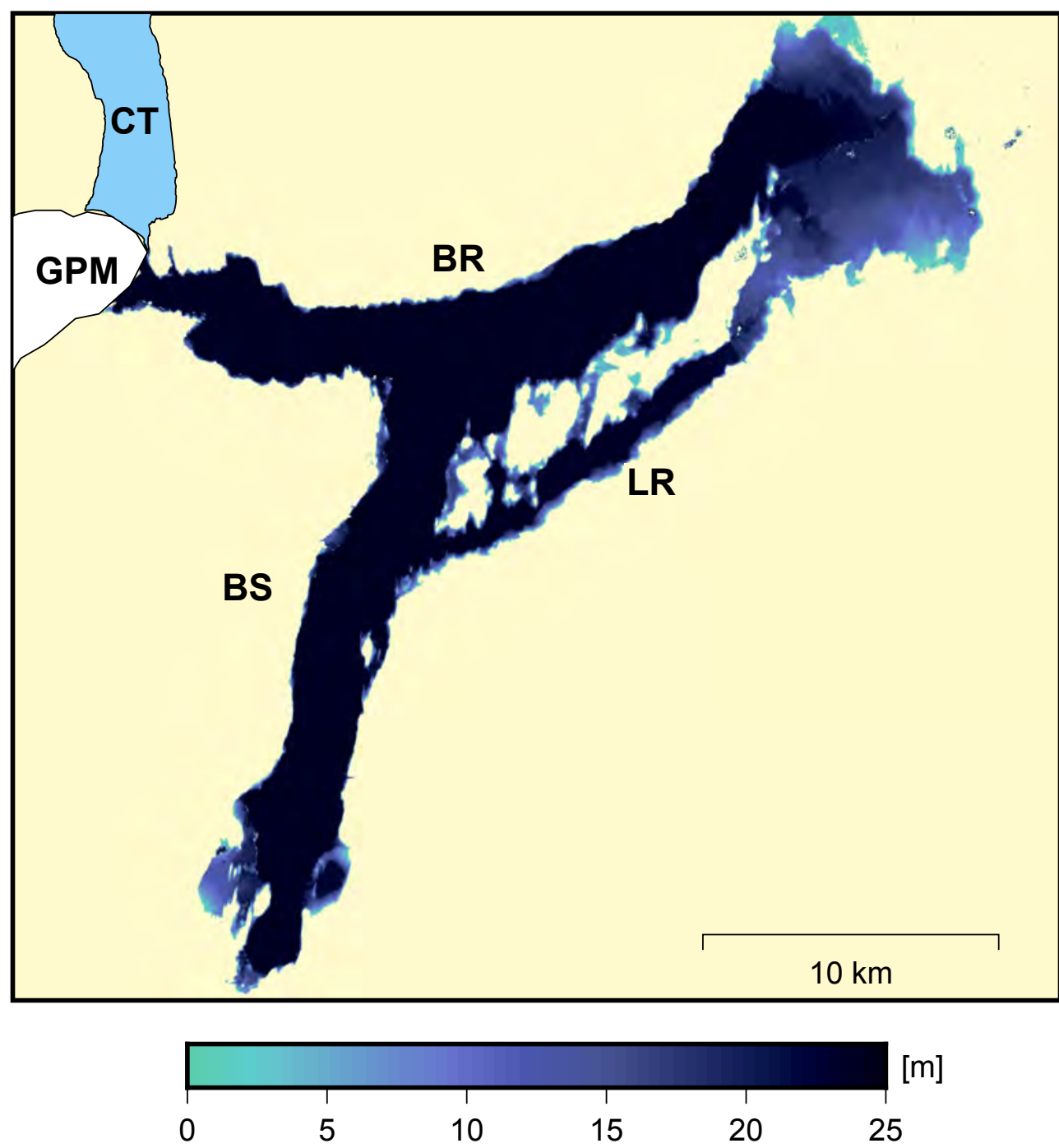

Figura 4.4: Modelo de carga correspondiente al endicamiento del glaciar Perito Moreno en los Brazos Rico y Sur. Representa el máximo semejante al registrado del año 1956.

\subsubsection{Represas del Río Santa Cruz}

En el valle del Río Santa Cruz, en el sur de la Provincia homónima, se iniciaron obras para la construcción de las represas hidroeléctricas Cóndor Cliff y Barrancosa. Una vez terminadas, acumularán niveles máximos de agua cercanos a los 65 y 40 metros, respectivamente. Esta acumulación de masa de agua como consecuencia del endicamiento representara una señal de carga superficial, a la cual responde la tierra sólida a través de una deformación elástica de la corteza terrestre y cambios locales del campo gravitatorio. Estas represas están siendo emplazadas en el kilómetro 250 para Cóndor Cliff y 185 para Barrancosa, definido desde la desembocadura del río Santa Cruz en el mar Argentino como kilometro cero (Massabie et al., 2007). Para el desarrollo del modelo de carga se utilizó un modelo digital de elevación elaborado por el Instituto Geográfico Nacional. Desarrollado con una resolución horizontal de 5 metros y una precisión vertical submetrica. 
Este comprende todo el área correspondiente al valle del Río Santa Cruz. El modelo de carga se construyó utilizando como cotas de referencia el pelo de agua de dichas represas los cuales corresponden a 176,5 msnm para Cóndor Cliff y $114 \mathrm{msnm}$ para Barrancosa (ACT EIA PRESAS SC, 2017) (las alturas msnm son determinadas como alturas elipsoidales y reducidas utilizando el modelo de geoide IGN).

El modelo IGN es un modelo aerofotogramétrico de alta precisión, el cual fue desarrollado con el fin de dar apoyo a las obras y definición de las cotas máximas del proyecto. Por sus características es muy superior en precisión a los modelos satelitales como SRTM (Shuttle Radar Topography Mission). Las alturas están dadas en alturas elipsoidales con marco de referencia geodésico POSGAR07. Este modelo digital de terreno se divide en tres sectores principales correspondientes a las fajas Gauss-Krüger 72, 69 y 66 grados de longitud Oeste. Para nuestro propósito se utilizaron los sectores 1 y 2 , correspondiente a las fajas 1 y 2 de 72 y 69 longitud Oeste, o equivalentemente sector Oeste y Este. Ambos debieron ser previamente revisados y corregidos. Se encontró una diferencia media de 6,01 metros entre los dos sectores mencionados. Esta diferencia se determinó para una zona reducida sobre las margenes del río correspondiente al solapamiento de las partes Oeste y Este del modelo, en la longitud de 71 grados Oeste (área de 0,1021 grados sexagesimales en longitud y 0,048 en latitud). Este sector del modelo comprende la zona del embalse de la represa Cóndor Cliff. El valor correspondiente a dicha diferencia fue utilizado para llevar ambas partes del modelo (Oeste y este) a un mismo nivel, las cuales fueron a su vez contrastadas contra un SRTM de 1 arco de segundo (USGS, 2016) para determinar los desvíos usándolo como nivel de base. Se encontró un buen acuerdo entre el sector Oeste correspondiente a la faja 1 y el SRTM, no así con la faja 2. Esta discrepancia no se llega a explicar con diferencias en el modelo de geoide, por lo que posiblemente se deba principalmente a una falta de ajuste a los puntos de control del sector Este del modelo del IGN. Además, valores atípicos ubicados sobre el lecho del río debieron ser eliminados y rellenados por interpolación. Como resultado, partiendo del modelo aerofotogramétrico del IGN se obtuvieron dos modelos de carga separados para cada represa con una resolución espacial de 5 metros, resultando en 67.714.441 de nodos en total, entre ambos (ver grafico 4.5). Esto representa 1,4 GB para el modelo de carga de la represa Cóndor Cliff y 859,4 MB para el modelo de carga de la Barrancosa. 


\subsection{Carga glaciar}

\section{Carga glaciar: Campos de Hielo Patagonico}

Los Campos de Hielo Patagónico Norte (HPN; 4.000 km2) y Sur (HPS; 12.500 km2), la masa más grande de hielo templado en el hemisferio sur (Naruse and Aniya, 1992). Representa la contribución más grande al aumento eustatico del nivel del mar por unidad de área, por ejemplo, 0,105 \pm 0,011 mm/a, 0,067 $\pm 0,004 \mathrm{~mm} / \mathrm{a}$ y 0,059 $\pm 0,005 \mathrm{~mm} / \mathrm{a}$, según Rignot et al. (2003); Willis et al. (2012b); Foresta et al. (2018), respectivamente. Con una altitud promedio de 1355 msnm (Willis et al., 2012b), los campos de hielo están alineados en dirección norte-sur a lo largo de la cresta de los Andes. Estos están continuamente alimentados por los vientos provenientes del océano Pacifico cargados de humedad que colisionan con el cordón cordillerano, recibiendo precipitaciones de hasta 10 metros por año equivalente de agua (Casassa et al., 2002). Muchos de los glaciares del sector occidental terminan sobre el nivel del mar con lenguas flotantes, mientras que en el sector oriental terminan mayormente en lagos (Casassa et al., 2012; Warren and Aniya, 1999).

Richter et al. (2019) hacen uso la misión satelital GRACE (Gravity Recovery and Climate Experiment), serie de tiempo mensuales de Nivel 2, para determinar los cambios en la masa de hielo de los Campos de Hielo Patagónico. El estudio comprende 15 años de la evolución de estas masas de hielo extrapolar, proporcionando nuevos indicios sobre la perdida de masa de hielo actual en altas latitudes en Patagonia. Esta evolución se encuentra dominada por una fuerte tendencia lineal relativamente ininterrumpida. Distintas correcciones debieron aplicarse en la determinación y ajuste a esta región, comprendiendo por un lado, el ajuste glacio-isostático explicado por un modelo regional que ha sido acotado a las observaciones realizadas con los sistemas globales de navegación por satélite (GNSS) (Lange et al., 2014). Corrección que debe ser previamente ajustada, removiendo la contribución elástica de acuerdo a un patrón de cambio de masa actual previamente establecido, como modelo de carga (Willis et al., 2012a,b), y las funciones de Green como modelo de Tierra (Farrell, 1972). Por otro lado, correcciones en relación al efecto de las variaciones de masa en el océano, en el almacenamiento de agua continental y en la capa de hielo antártico. Las 161 soluciones mensuales del campo gravitacional de GRACE se invierten en el dominio espacial mediante el ajuste de los factores de escala aplicados a los patrones de cambio de masa de hielo a-priori basados en los resultados de teledetección publicados para los Campos de Hielo Sur y Norte de la Patagonia, respectivamente (Willis et al., 2012a,b). Espacialmente de GLIMS (Global Land Ice Measurements from Space) se obtienen los contornos glaciares donde se introducen los mencionados patrones de variación de las masas de hielo. De este trabajo se determinó una tasa de cambio de la masa de hielo de $-24.4 \pm 4.7$ Gt/a para los Campos de Hielo Patagónicos entre abril de 2002 y junio de 2017, lo que corresponde a una contribución al aumento del nivel del mar de $0.067 \pm 0.013 \mathrm{~mm} / \mathrm{a}$, y una variación anual con amplitud de 55 Gigatoneladas (Richter et al., 2019). Sobre el trabajo de Richter et al. (2019) se puso un gran esfuerzo en determinar el aporte individual de los dos Campos de Hielo Patagónico a la perdida de masa total observada por GRACE. Esto revela un aporte inesperadamente grande del Campo de Hielo Patagónico Norte: sobre un cuarto del área, tiene lugar un poco más de 
tercio de la perdida de masa. Aporte que se ve reflejado en los resultados obtenidos del cálculo de efectos elásticos de carga glaciar presentados en este trabajo (capítulo ??). 


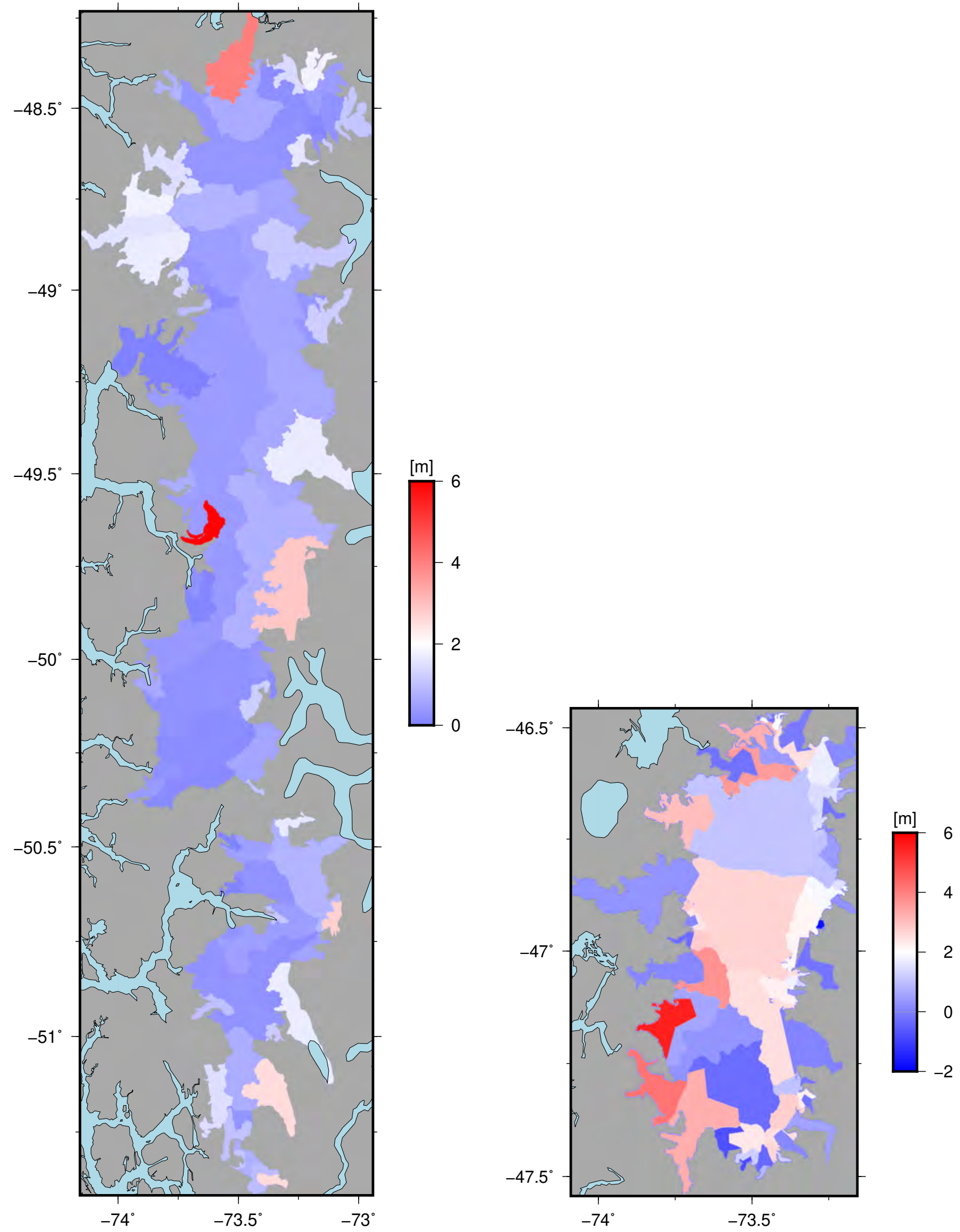

Figura 4.6: Campos de Hielo Patagónico Sur (derecha) y Norte (izquierda). Se muestra la taza de perdida de masa de hielo en metros por año para cada cuenca glaciar. 


\section{Capítulo 5}

\section{Resultados}

En este capítulo se presentan los resultados de la aplicación del programa desarrollado a los modelos de carga correspondientes según los procesos descriptos en el capítulo previo. En la evaluación e interpretación de la magnitud de los efectos se hace énfasis en la precisión alcanzable con métodos observacionales geodésicos (posicionamiento GNSS, gravimetría y otros) (ver tabla 5.1). Esto determina el punto de partida para la utilidad de los valores estimados de efectos de carga elásticos correspondiente a los distintos modelos de carga.

Las funciones de Green utilizadas para el cálculo de efectos de carga son las dadas por Farrell (1972) correspondiente al modelo de Tierra Gutenberg-Bullen-A, esto permitió la comparación de los modelos de carga oceánica con el calculador Ocean Tide Loading Provider (Bos and Scherneck, 2011), desarrollado en la sección 5.1. Debido a que la función de Green para el potencial no está dada por Farrell (1972), y por lo expresado en la sección 2.5 en cuanto a los efectos de carga para ambos modelos de Tierra, se resolvió utilizar las funciones de Green de potencial para el modelo PREM (Francis and Mazzega, 1990). Por otro lado, además de los efectos determinados por ELOADE (capítulo 2), la combinación de las deformaciones obtenidas con las funciones de Green correspondiente al modelo Gutenberg-Bullen-A y los efectos de carga para el potencial correspondiente al modelo PREM, es posible obtener estimaciones del cambio de altura ortométrica, y mediante cálculo de gradientes de la deformación vertical, valores estimados de inclinación (ver sección 2.3.4).

Uno de los motivos de estos cálculos es determinar ubicaciones óptimas de futuros puntos de medición destinados a la determinación observacional de los efectos de carga. Cabe resaltar que la selección de tales sitios no solo tiene que proveer de las máximas magnitudes de los observables, sino también garantizar el acceso para las técnicas de observación geodésica, y por lo tanto, es evidente deben restringirse a los sitios ubicados en tierra. 
Tabla 5.1: Métodos de observación geodésicos

\begin{tabular}{llr}
\hline Efecto & Técnica de observación & Magnitud precisión alcanzable \\
\hline $\begin{array}{l}\text { Desplazamiento } \\
\text { Cambios de inclinación }\end{array}$ & GNSS & $5 \mathrm{~mm}$ (Altamimi et al., 2016) \\
& & $2,5 \mathrm{nrad}$ (resolución) \\
Cambios de gravedad & Gravímetro absoluto & (UNAVCO, 2020; Agnew, 1986) \\
Cambios de tensión & Extensómetro láser & $\begin{array}{r}10^{-9} \text { (Agnel (Sasagawa et al., 1995) } \\
\text { Deformación }\end{array}$ \\
InSAR & $<1 \mathrm{~cm}$ (Gabriel et al., 1989)
\end{tabular}

\subsection{Efectos de carga oceánica}

Se presentan los resultados correspondientes a los modelos de marea oceánica, EOT11a y la combinación de éste con el modelo de marea obtenido por D'onofrio et al. (2016) para la Isla Grande de Tierra del Fuego. Se pretende obtener una estimación de la calidad del cálculo para ELOADE de efectos de carga, contrastando con valores calculados provistos por Bos and Scherneck (2011). Ambos para los mismos modelos de entrada, tanto modelo de Tierra como de carga.

\subsubsection{Predicciones por Bos and Scherneck (2011) - Modelo EOT11a}

Con el objetivo de validar nuestro código para el cálculo de efectos de carga, se calcularon los efectos para una seria de puntos correspondientes a 44 sitios de observación, dedicados estos al estudio del ajuste glacio-isostático del Campo de Hielo Patagónico Sur, ubicados entre Argentina y Chile según la figura 5.1 para las ocho principales componentes de marea diurnas y semi-diurnas (M2, S2, N2, K2, K1, O1, P1 y Q1) del modelo de marea oceánica global empírico EOT11a (Savcenko et al., 2012). Para tal fin, los cálculos con ELOADE se realizaron utilizando las componentes real e imaginaria, para luego transformar a amplitud y fase. Estos resultados fueron comparados con los entregados por el calculador Ocean Tide Loading Provider (Bos and Scherneck, 2011) de la Universidad de Chalmers, Suecia. En la tabla 5.2 se muestran los valores obtenidos para la deformación vertical de la componente semi-diurna M2 calculados por ELOADE y sus diferencias absolutas para las amplitudes y fases, con las calculadas por Bos and Scherneck (2011). La tabla 5.1.1 resume las diferencias para las ocho componentes de marea utilizadas. Se determinaron las diferencias medias de cada componente para todos los puntos mencionados mediante la raíz de la media cuadrática aplicada a las diferencias absolutas.

Tabla 5.2: Comparación componente semi-diurna M2. 


\begin{tabular}{|c|c|c|c|c|c|c|}
\hline $\begin{array}{l}\text { Punto } \\
\text { Nro. }\end{array}$ & $\begin{array}{c}\text { Longitud } \\
\left.{ }^{\circ}\right]\end{array}$ & $\begin{array}{c}\text { Latitud } \\
{\left[{ }^{\circ}\right]}\end{array}$ & $\begin{array}{r}\text { Amplitud } \\
{[\mathrm{mm}]}\end{array}$ & $\begin{array}{r}\text { Fase } \\
{\left[{ }^{\circ}\right]}\end{array}$ & $\begin{array}{r}\Delta \\
{[m m]}\end{array}$ & $\begin{array}{c}\Delta \\
{\left[{ }^{\circ}\right]}\end{array}$ \\
\hline 1 & $-69,5299$ & $-50,2453$ & 13,015 & $-155,517$ & 0,035 & 0,383 \\
\hline 2 & $-72,1421$ & $-50,0048$ & 2,012 & 168,811 & 0,002 & 0,311 \\
\hline 3 & $-72,9529$ & $-50,6573$ & 1,206 & 126,021 & $-0,024$ & 1,621 \\
\hline 4 & $-73,6277$ & $-50,6365$ & 1,173 & 58,772 & $-0,087$ & 2,972 \\
\hline 5 & $-72,4852$ & $-50,3468$ & 1,672 & 156,647 & $-0,008$ & 0,547 \\
\hline 6 & $-73,2524$ & $-50,3518$ & 0,840 & 95,995 & $-0,030$ & 3,195 \\
\hline 7 & $-72,9918$ & $-49,5164$ & 0,543 & 118,034 & $-0,007$ & 2,934 \\
\hline 8 & $-72,8648$ & $-49,6661$ & 0,733 & 134,814 & $-0,007$ & 2,014 \\
\hline 9 & $-70,6522$ & $-50,2840$ & 6,452 & $-167,272$ & $-0,048$ & 0,028 \\
\hline 10 & $-73,1819$ & $-49,9062$ & 0,629 & 99,848 & $-0,031$ & 3,548 \\
\hline 11 & $-72,8938$ & $-50,8362$ & 1,377 & 128,335 & $-0,023$ & 1,335 \\
\hline 12 & $-73,2341$ & $-50,1979$ & 0,760 & 97,001 & $-0,030$ & 3,601 \\
\hline 13 & $-70,7898$ & $-50,2237$ & 5,775 & $-168,789$ & $-0,015$ & 0,111 \\
\hline 14 & $-68,4009$ & $-50,1436$ & 32,114 & $-142,542$ & 3,874 & 1,658 \\
\hline 15 & $-72,8536$ & $-49,9253$ & 0,892 & 137,445 & $-0,008$ & 1,745 \\
\hline 16 & $-72,9122$ & $-49,0986$ & 0,405 & 112,962 & $-0,015$ & 3,262 \\
\hline 17 & $-69,2087$ & $-51,6480$ & 23,746 & $-163,855$ & 0,156 & 0,245 \\
\hline 18 & $-71,6425$ & $-50,3254$ & 3,381 & 178,603 & $-0,009$ & 0,203 \\
\hline 19 & $-72,9902$ & $-48,4250$ & 0,351 & 35,485 & $-0,029$ & $-0,115$ \\
\hline 20 & $-73,1425$ & $-49,3072$ & 0,406 & 83,115 & $-0,024$ & 4,015 \\
\hline 21 & $-74,1459$ & $-50,8348$ & 2,009 & 31,602 & $-0,171$ & 0,802 \\
\hline 22 & $-73,0283$ & $-48,9972$ & 0,334 & 83,095 & $-0,016$ & 3,195 \\
\hline 23 & $-73,7093$ & $-50,6292$ & 1,252 & 52,169 & $-0,098$ & 2,569 \\
\hline 24 & $-73,7011$ & $-50,4972$ & 1,168 & 50,483 & $-0,092$ & 2,783 \\
\hline 25 & $-72,9731$ & $-48,0553$ & 0,473 & 15,390 & $-0,027$ & $-0,210$ \\
\hline 26 & $-73,9212$ & $-49,5211$ & 1,164 & 15,317 & $-0,126$ & 0,517 \\
\hline 27 & $-73,4541$ & $-49,5992$ & 0,616 & 49,393 & $-0,064$ & 2,893 \\
\hline 28 & $-74,0871$ & $-49,2458$ & 1,386 & 3,075 & $-0,154$ & 1,075 \\
\hline 29 & $-73,1343$ & $-48,9309$ & 0,355 & 55,208 & $-0,035$ & 1,508 \\
\hline 30 & $-73,2479$ & $-50,9920$ & 1,271 & 95,576 & $-0,039$ & 2,776 \\
\hline 31 & $-73,5831$ & $-50,4593$ & 1,030 & 59,872 & $-0,070$ & 3,572 \\
\hline 32 & $-73,0464$ & $-48,7020$ & 0,327 & 50,585 & $-0,023$ & 1,485 \\
\hline 33 & $-72,7404$ & $-48,8675$ & 0,423 & 124,528 & $-0,007$ & 2,228 \\
\hline 34 & $-72,5996$ & $-51,5837$ & 2,169 & 131,742 & $-0,001$ & 1,642 \\
\hline 35 & $-73,3527$ & $-49,1663$ & 0,498 & 39,285 & $-0,052$ & 2,585 \\
\hline 36 & $-72,5941$ & $-48,4847$ & 0,366 & 116,827 & $-0,004$ & 2,527 \\
\hline 37 & $-74,4090$ & $-49,1262$ & 1,877 & $-5,950$ & $-0,163$ & 0,450 \\
\hline 38 & $-73,1388$ & $-49,1585$ & 0,373 & 73,371 & $-0,027$ & 3,071 \\
\hline 39 & $-72,4438$ & $-48,2654$ & 0,390 & 116,637 & 0,000 & 2,337 \\
\hline 40 & $-73,1398$ & $-49,1601$ & 0,374 & 73,301 & $-0,026$ & 3,101 \\
\hline
\end{tabular}




\begin{tabular}{rccrrrr}
\hline $\begin{array}{r}\text { Punto } \\
\text { Nro. }\end{array}$ & $\begin{array}{c}\text { Longitud } \\
\left.{ }^{\circ}\right]\end{array}$ & $\begin{array}{c}\text { Latitud } \\
{\left[{ }^{\circ}\right]}\end{array}$ & $\begin{array}{r}\text { Amplitud } \\
{[\mathrm{mm}]}\end{array}$ & $\begin{array}{r}\text { Fase } \\
{\left[{ }^{\circ}\right]}\end{array}$ & $\begin{array}{r}\Delta \\
{[\mathrm{mm}]}\end{array}$ & $\begin{array}{r}\Delta \\
{\left[^{\circ}\right]}\end{array}$ \\
\hline 41 & $-72,8343$ & $-51,3166$ & 1,739 & 123,684 & $-0,001$ & 2,084 \\
42 & $-72,9519$ & $-51,1780$ & 1,542 & 118,107 & $-0,018$ & 2,007 \\
43 & $-73,2797$ & $-51,1190$ & 1,375 & 91,642 & $-0,045$ & 2,942 \\
44 & $-73,2896$ & $-49,3714$ & 0,463 & 60,520 & $-0,047$ & 3,320 \\
\hline
\end{tabular}

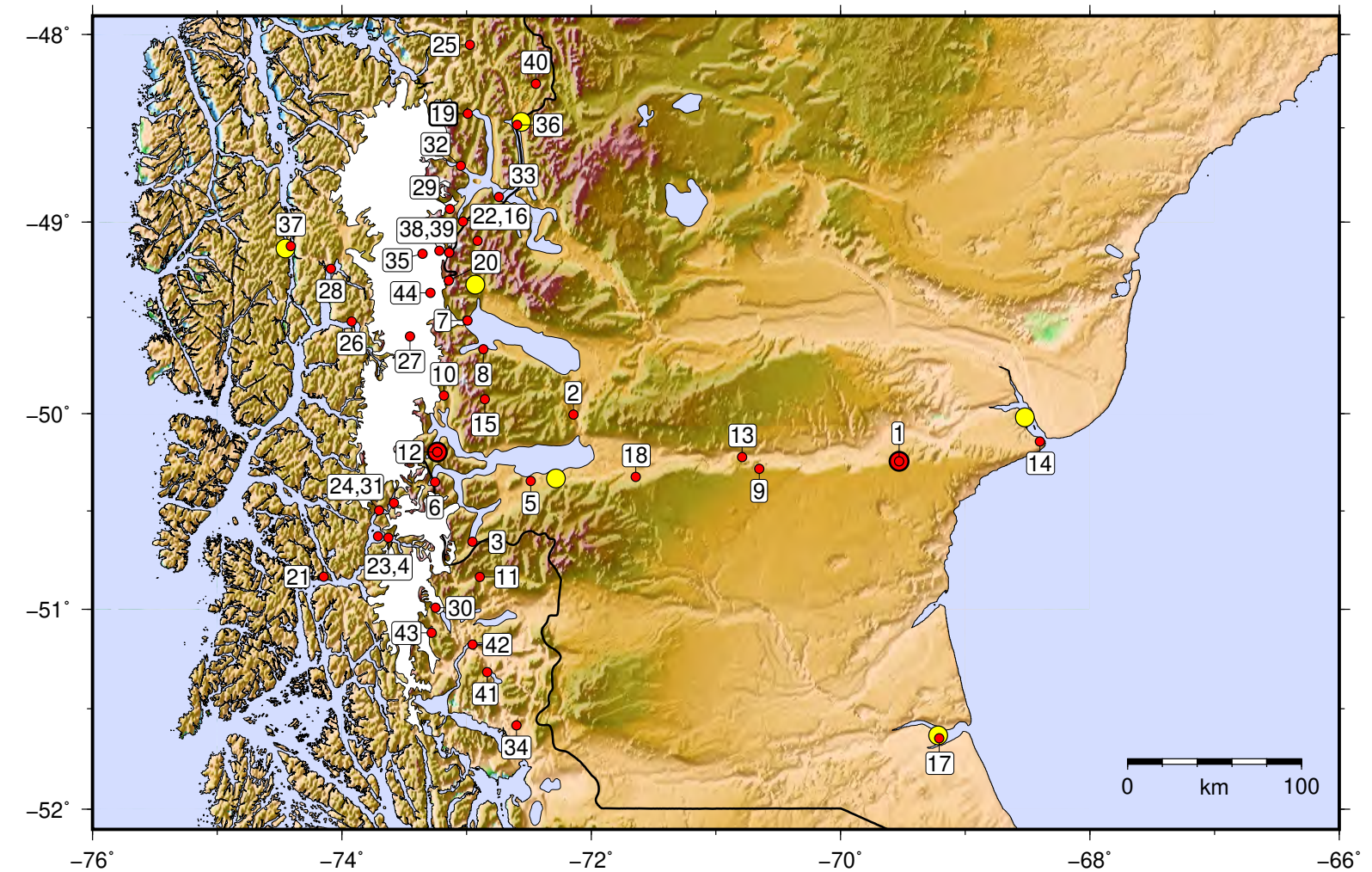

Figura 5.1: Puntos utilizados en la comparación de los efectos de carga con Bos and Scherneck (2011). Estos mismos puntos corresponden a los sitios de observación GNSS creados para el estudio de ajuste glacio-isostático (Richter et al., 2016). Los círculos amarillos indican las localidades de Puerto Eden y Villa O'Higgins en Chile y El Chalten, El Calafate, Puerto Santa Cruz y Río Gallegos en Argentina.

De la tabla 5.2 se observa en general un buen acuerdo entre los valores obtenidos por ambos códigos. Para el punto número 14, ubicado sobre la costa próximo a Puerto Santa Cruz, se obtuvo una diferencia absoluta cercana a los $4 \mathrm{~mm}$, esto representa una diferencia relativa de $12 \%$. Esta diferencia se explica para este punto por la metodología utilizada por Bos and Scherneck (2011) en el cálculo sobre las zonas costeras. Dicho cálculo involucra un gran detalle de la línea de costa que es utilizado para aplicar distintos niveles de resolución en la interpolación de la carga cercana. En cambio, ELOADE aplica una interpolación bilineal, cuya cantidad de celdas (o elementos cercanos) utilizadas en la interpolación dependen de la resolución del modelo de carga, en el caso del EOT11a alrededor de $13 \mathrm{~km}$ $\left(0.125^{\circ}\right)$, manteniendo siempre la misma cantidad de elementos a interpolar sobre una subdivisión radial de estos. Una mayor distancia a la carga disminuye la magnitud del 
efecto y, por tanto, las diferencias debidas a los distintos métodos de interpolación. Esta explicación confirma el resultado del punto 17 de la tabla 5.2, ubicado en Río Gallegos a unos $20 \mathrm{~km}$ dentro del estuario, donde la diferencia entre ambos cálculos no alcanza los 0,2 $\mathrm{mm}$ (menor al 1\%). Diferencias encontradas sobre puntos lejanos a la carga que representan una deformación muy pequeña (submilimétrica), podrían tener diferencias relativas grandes. Esto podría deberse a las diferencias en la representación computacional utilizada en los cálculos, así también como errores de redondeo o incluso por el orden de las operaciones por diferencias en los algoritmos o funciones utilizadas.

Tabla 5.3: Comparación de los resultados obtenidos con ELOADE y Bos and Scherneck (2011) para la deformación vertical de las componentes semi-diurnas y diurnas más importantes. $\mathrm{R}$ es el promedio de los módulos de las diferencias relativas. Los valores se calcularon sobre los 44 puntos indicados en la figura 5.1.

\begin{tabular}{cccr}
\hline Componente & RMS [mm] & ${\text { RMS }\left[^{\circ}\right]}^{\text {R [\%] }}$ \\
\hline M2 & 0,581 & 2,32 & 4,74 \\
K1 & 0,083 & 0,37 & 1,02 \\
K2 & 0,046 & 1,35 & 1,04 \\
S2 & 0,152 & 0,58 & 2,10 \\
N2 & 0,149 & 2,63 & 2,64 \\
O1 & 0,089 & 0,22 & 1,07 \\
P1 & 0,030 & 0,35 & 1,09 \\
Q1 & 0,017 & 0,12 & 0,87 \\
\hline
\end{tabular}

\subsubsection{Predicciones - ELOADE, modelo EOT11a}

Se calculó la deformación vertical y fases para las principales componentes de marea oceánica sobre una cuadrícula correspondiente a toda la región de la Patagonia austral. Partiendo de componentes real e imaginaria se calculan las deformaciones que nuevamente se transforman en amplitud y fase. La figura 5.2 muestra el modelo de marea EOT11a componente más importante semi-diurna M2 y la amplitud y fases para el efecto de deformación vertical.

Los resultados del modelado (ver figura 5.2) sugieren que las deformaciones verticales responden al patrón de mareas oceánicas. En las fases se observa una circulación antihoraria en la deformación alrededor de un punto anfidrómico a una latitud de Puerto Deseado y a una longitud aproximada de Caleta Olivia. De este punto parten líneas de co-fase con forma curva, una de ellas se observa que corresponde a la discontinuidad del ángulo de fase de $\pm 180^{\circ}$. Las fases indicadas a lo largo de esta línea visible se explican como un artefacto debido a los efectos de interpolación y redondeo a través de esta línea al gratificar la imagen. Las amplitudes máximas de los efectos se encuentran a lo largo de la costa atlántica, donde se concentran las máximas amplitudes de marea. La deformación vertical calculada para la componente M2 no supera los $35 \mathrm{~mm}$ a lo largo de la costa para dicha componente. 

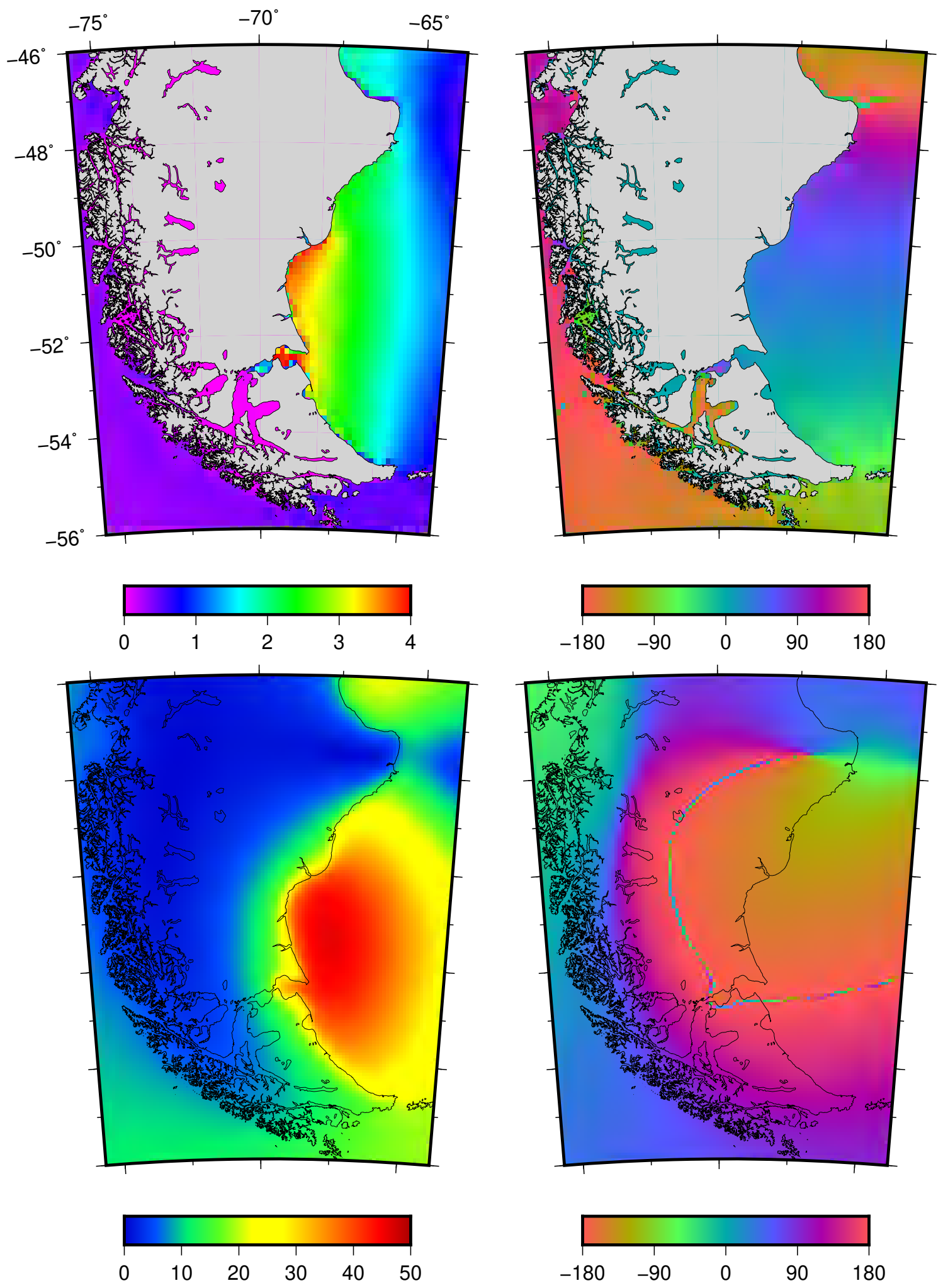

Figura 5.2: Carga oceánica (arriba, amplitudes en $m$ a la izquierda, fases de retardo relativo al meridiano de Greenwich en grados a la derecha) y deformación vertical resultante del efecto de carga (abajo, amplitudes en $\mathrm{mm}$ a la izquierda y fases en grados a la derecha) para la componente semi-diurna M2 correspondiente al modelo EOT11a. 


\subsubsection{Modelo Regionalizado: EOT11a más D’onofrio et al. (2016)}

Sobre los cálculos del modelo combinado EOT11a con un modelo local D'onofrio et al. (2016) (ver Capítulo 4) se pueden observar diferencias significativas sobre la costa de Tierra del Fuego. Un rasgo importante a observar en los mapas de la figura 5.4 correspondiente a los efectos del modelo combinado, es de notar la unión de ambos modelos (línea correspondiente al límite norte del modelo D'onofrio et al. (2016)). Esto se debe a que el cálculo se ejecutó de manera independiente con el objetivo de mantener la resolución espacial. Dado que ELOADE realiza interpolaciones sobre las masas cercanas, esto conlleva a que se observen efectos de borde en los modelos. Estos efectos de borde que se observa en la imagen de la figura 5.4 pueden ser resuelto de diferentes maneras, siendo un suavizado la más práctica.

En la figura 5.3 se calcularon las diferencias de los efectos de carga determinados por ambos modelos, EOT11a y el combinado con D'onofrio et al. (2016). Concentrándonos en la Isla Grande de Tierra del Fuego (dominio del modelo de D'onofrio et al. (2016)), se observa sobre la costa diferencias absolutas que están entre los -2 y $4 \mathrm{~mm}$. Diferencias que son significativas para observaciones GNSS.

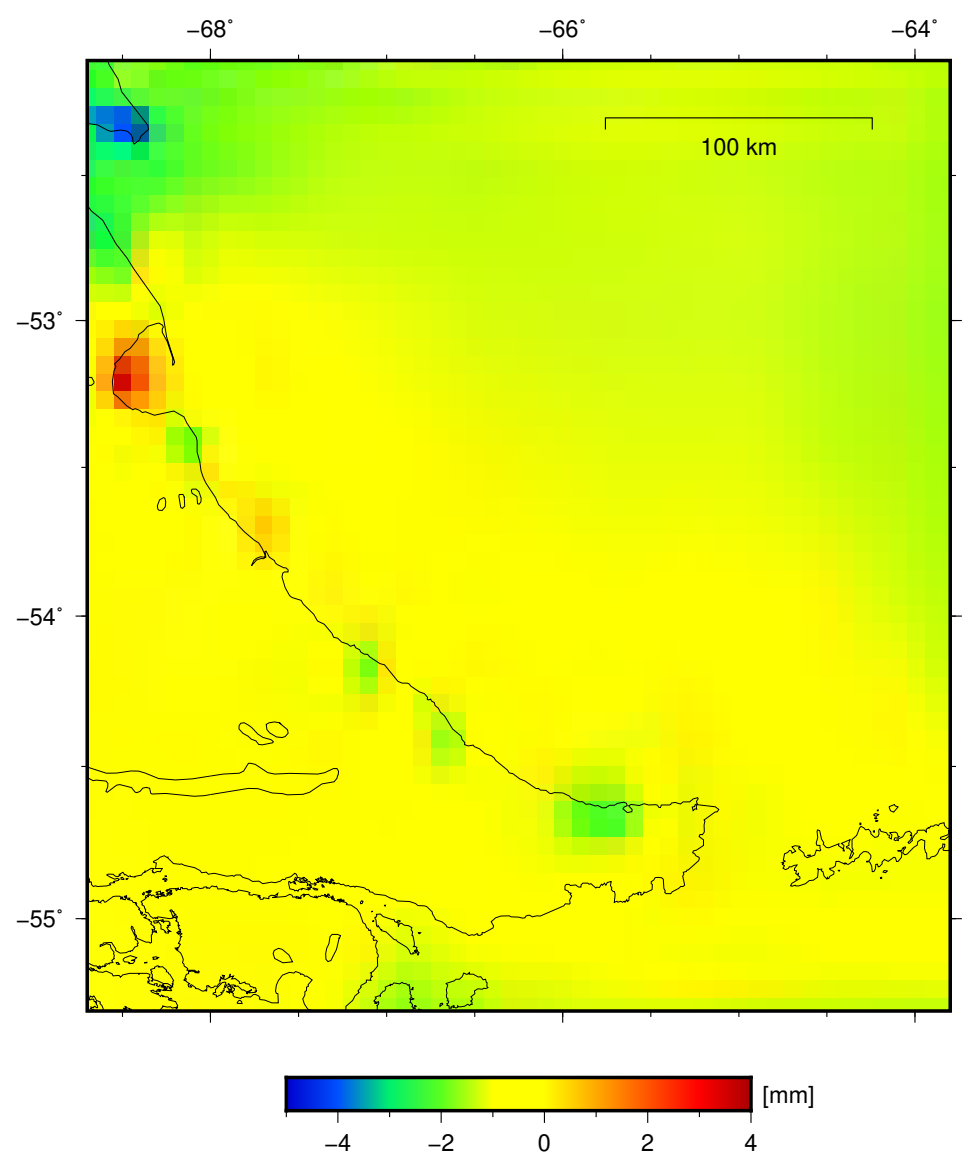

Figura 5.3: Diferencias entre las amplitudes de deformación vertical para la componente semi-diurna M2 entre el cálculo aplicando el modelo global EOT11a y el modelo regionalizado utilizando D'onofrio et al. (2016). 

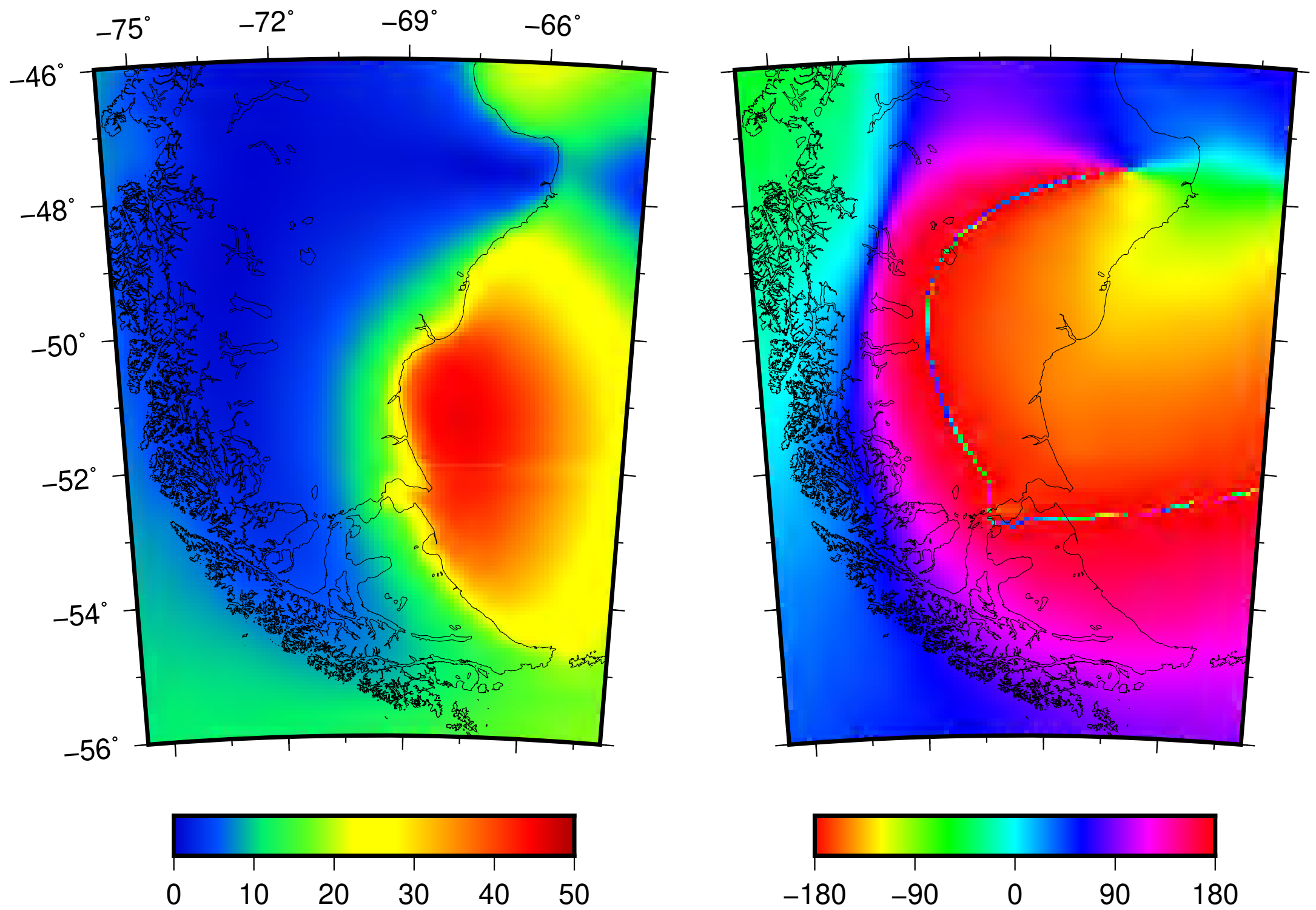

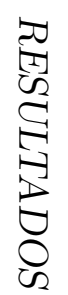

Figura 5.4: Efecto de carga oceánica del modelo regionalizado para la componente M2. Amplitud en $\mathrm{mm}$ (derecha) y fase en grados (izquierda) 


\subsubsection{Efectos de carga oceánica no-mareal}

La herramienta de cálculo utilizada (ELOADE) permite el cálculo de efectos de carga oceánica no-mareal. Esto requiere la introducción de modelos de carga adecuados. Información proveniente de altimetría satelital y mareógrafos costeros podrían utilizarse para estos fines. Por otro lado, se requiere una discretización en tiempo a fin de evaluar su respuesta. En la práctica se compilan modelos de carga diferencial entre dos épocas (por ejemplo: dos épocas de observación geodésica). Sin embargo, la magnitud de las variaciones no maréales del nivel del mar en la zona de estudio es pequeña. Esta se encuentra en el orden de decímetros (Richter et al., 2012b), un orden de magnitud menor en comparación con las amplitudes de marea oceánica, la cual se encuentra en el orden de metros (Glorioso and Flather, 1997). Debido a esto es de esperar que los aportes no sean significativos.

\subsection{Efectos de carga atmosférica}

\subsubsection{Ciclo anual y diurno}

Sobre la base de los datos sinópticos (Servicio Meteorológico Nacional, 2014) utilizados en la construcción de los modelos atmosféricos de carga (ver figura 4.1, sección ??) se presentan las figuras de los modelos de carga y la deformación vertical obtenidos mediante el código ELOADE. En la figura 5.5 se pueden observar a la izquierda, amplitudes y fases de los modelos de carga, y a la derecha, amplitudes y fases de sus efectos. De arriba hacia abajo se ordenan las componentes diurna, semi-diurna, anual y semi-anual, respectivamente. Como es de esperar, las mayores deformaciones se concentran hacia la región interna continental, con mayor lejanía al océano, donde la compensación oceánica es menos efectiva. De esta información se desprende que las amplitudes se encuentran muy por debajo de las precisiones alcanzables por métodos geodésicos (GNSS, tabla 5.1). Esto mismo sugeriría que el uso de modelos de carga atmosférica aplicados en calidad de corrección a las observaciones, no introducirían una mejora sustancial en los resultados observacionales en esta región.

\subsubsection{Evento de cambio abrupto de presión atmosférica}

En términos de amplitud de presión atmosférica, eventos esporádicos pueden alcanzar magnitudes en un orden mayor que las variaciones periódicas descriptas anteriormente. Sin embargo los efectos de carga no alcanzan magnitudes significativas. Eso se debe a que la corteza terrestre actúa como un filtro pasa bajo y la longitud de onda espacial de estas anomalías atmosféricas son relativamente cortas. La figura 5.6 muestra el evento atmosférico mencionado en la sección 4.2, donde se puede observar el desplazamiento del frente de baja presión en dirección oeste este. 

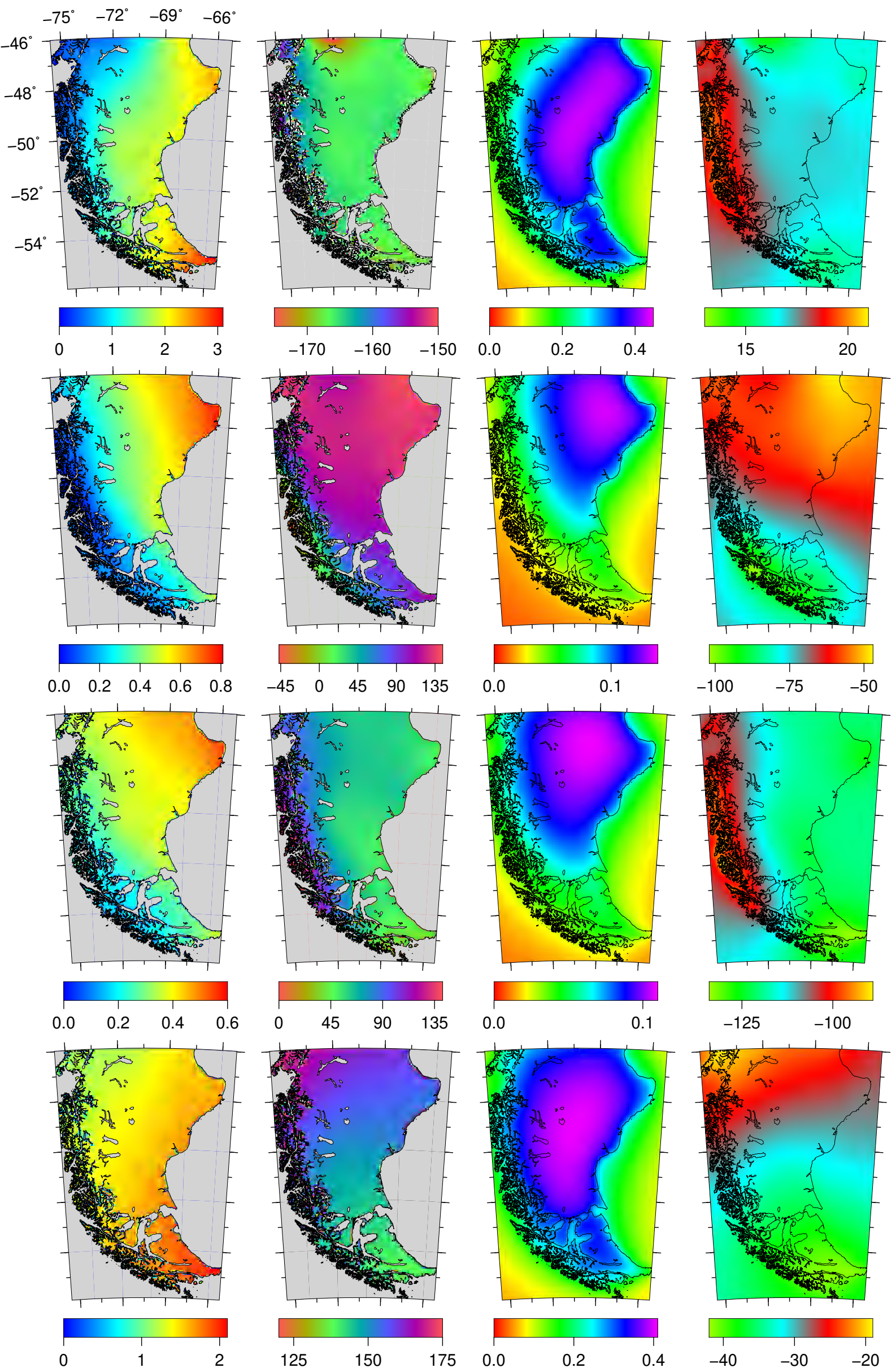

Figura 5.5: Carga atmosférica y efectos de carga, izquierda y derecha, respectivamente. Amplitudes de los modelos de carga se muestran en $h P a$. Amplitudes $[\mathrm{mm}]$ y fases $\left[^{\circ}\right]$ de las componentes diurna, semi-diurna, anual y semi-anual, de arriba hacia abajo, respectivamente para los modelos de deformación vertical. 

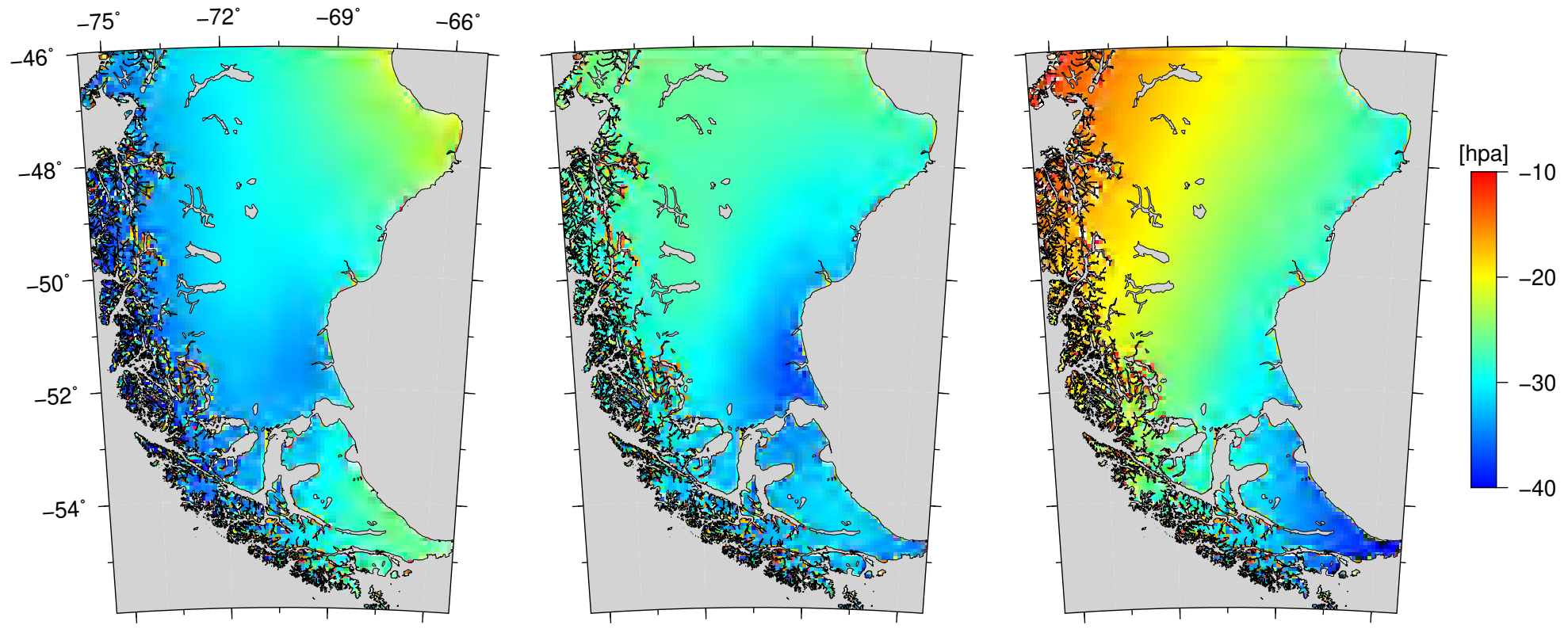

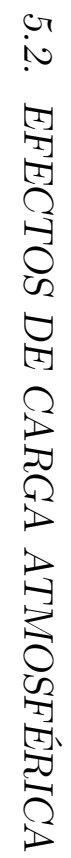
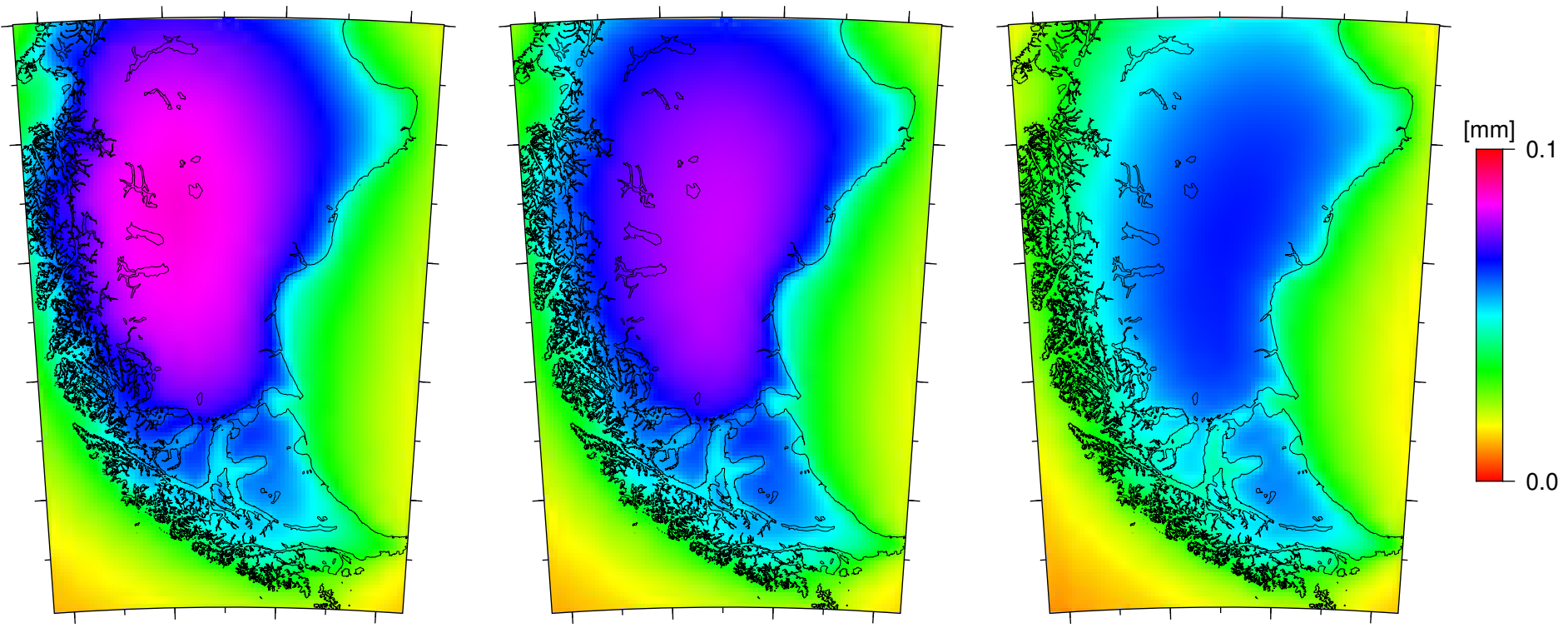

Figura 5.6: Evento atmosférico. Se muestra de derecha a izquierda a las 17, 19 y 22 horas UTC. Arriba se representa el modelo de carga atmosférica para tres pasos temporales. Abajo se muestran los efectos de carga atmosférica según los pasos temporales descriptos. 


\subsection{Efectos de carga hidrológica}

\subsubsection{Los grandes lagos patagónicos}

De los modelados independientes de cada lago se determinó un modelo de efectos de carga correspondiente a los grandes lagos. Un mapa de la deformación vertical se muestra en la figura 5.7. Se observan deformaciones cuya magnitud supera el nivel de ruido de observación GNSS. Las deformaciones máximas se concentran en los lagos cercanos al campo de hielo sur. Ésta puede afectar la determinación en la componente elástica del ajuste glacio-isostático en la región si no es tenida en cuenta. Como los efectos están controlados por la variación estacional del nivel de los lagos, su magnitud depende de la época de observación. Esto sugiere que mediciones dedicadas a la observación de deformaciones causados por otros procesos deberían llevarse a cabo en el mismo periodo del año o aplicar correcciones basados en modelos como el presentado (ver figura 5.7).

Se ha iniciado un proyecto de gravedad absoluta (Scheinert and Rulke, 2018) dedicado al estudio del ajuste glacio-isostático. Las observaciones de gravedad absoluta requieren correcciones por el efecto gravimétrico de carga hidrológica en los lagos próximos a los puntos de medición. Con este fin se prevén observaciones in-situ del nivel del agua en simultáneo a las mediciones gravimétricas. Se aplicará el código ELOADE para el cálculo de estas correcciones. En la figura 5.8 se muestran resultados del modelado de efectos de carga para la gravedad, cuyo modelo de carga introducido corresponde al de cambio de nivel de agua de los grandes lagos, por lo que de esta manera se proporcionan valores preliminares. 


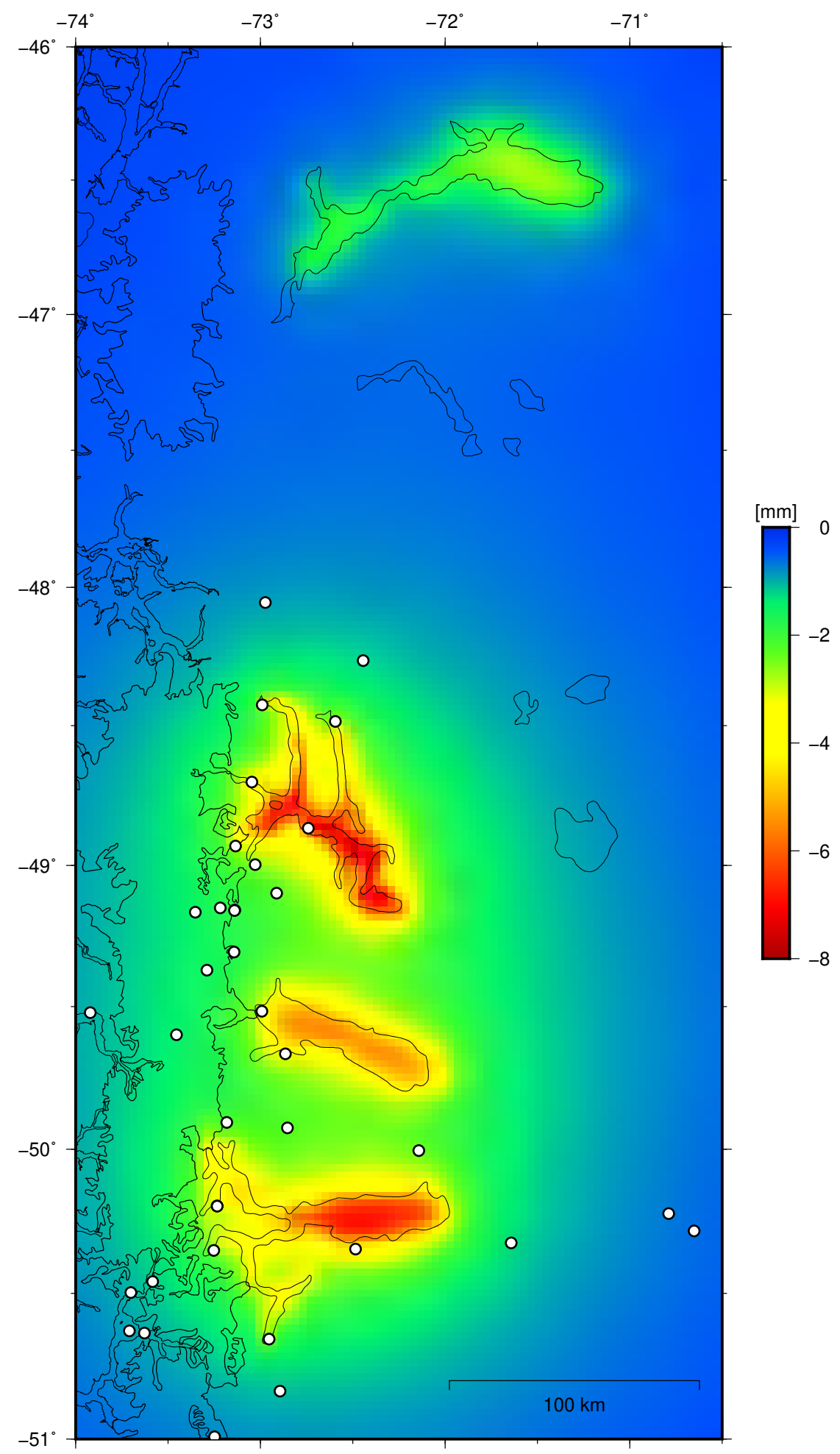

Figura 5.7: Efecto de carga para los grandes lagos correspondiente a la componente vertical. De norte a sur los lagos son según Argentina/Chile: Buenos Aires/General Carrera, San Martin/OHiggins, Viedma y Argentino. Los puntos blancos indican los sitios de observación GNSS dedicados al estudio del ajuste glacio-isostático (Richter et al., 2016). 


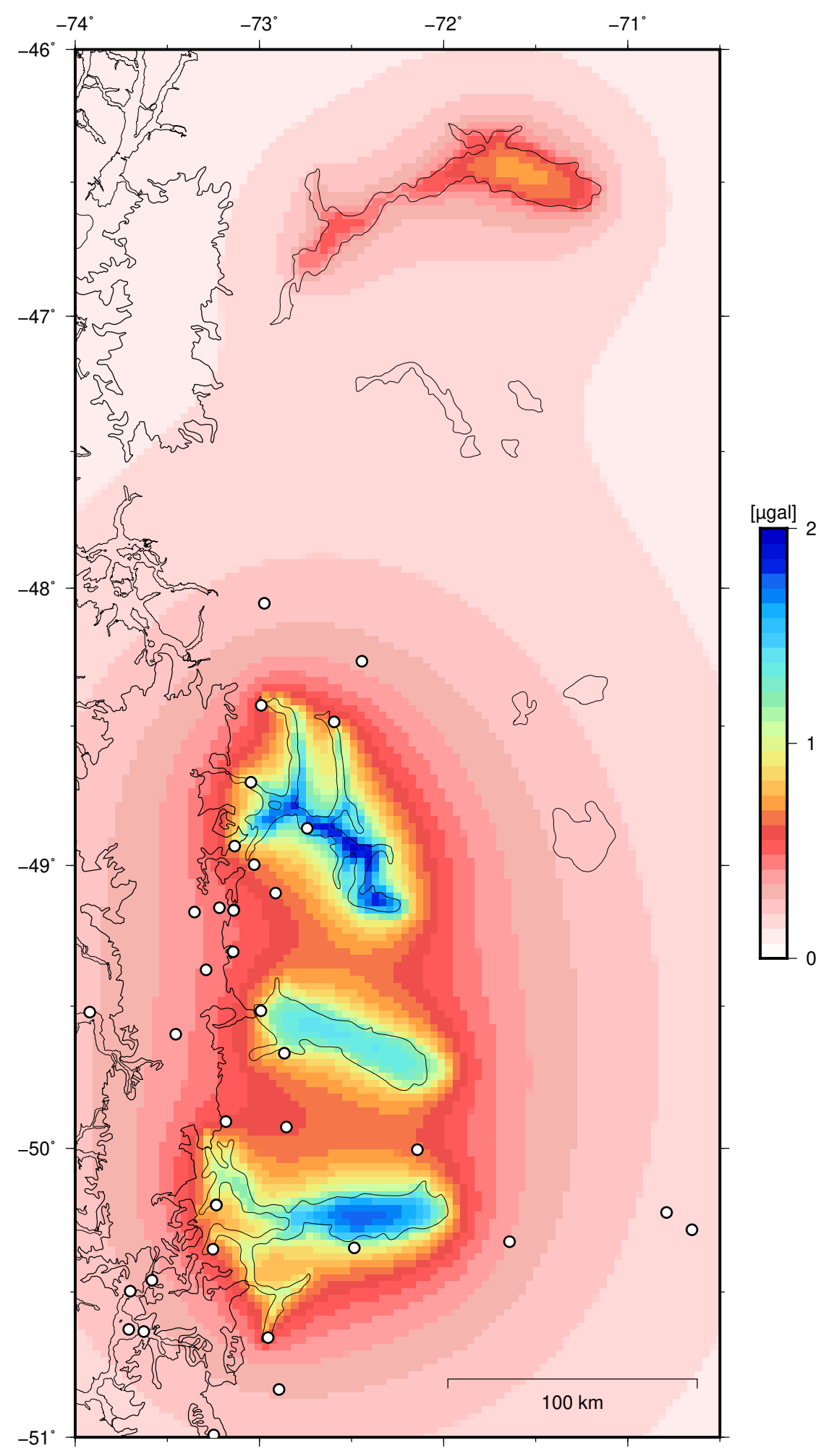

Figura 5.8: Efecto de carga para los grandes lagos correspondiente al cambio de gravedad. De norte a sur los lagos son según Argentina/Chile: Buenos Aires/General Carrera, San Martin/OH́iggins, Viedma y Argentino. Los puntos blancos indican los sitios de observación GNSS dedicados al estudio del ajuste glacio-isostático (Richter et al., 2016). 


\subsubsection{Brazo Rico/Sur}

De los modelos de carga derivados para los distintos endicamientos producidos por el glaciar Perito Moreno, se muestran los resultados correspondientes al rompimiento del frente del glaciar para la carga máxima estimada de los años 2016 y 1956 (ver tabla 4.2). Conjuntamente se calculó el efecto de la masa de agua correspondiente para la compensación sobre el lago Argentino al momento del rompimiento y la descarga del embalse hacia el lago. De estos resultados se ubicaron nuevos sitios para futuras observaciones. Sobre cada modelo de efectos se identifica la línea de costa para el correspondiente modelo de carga.

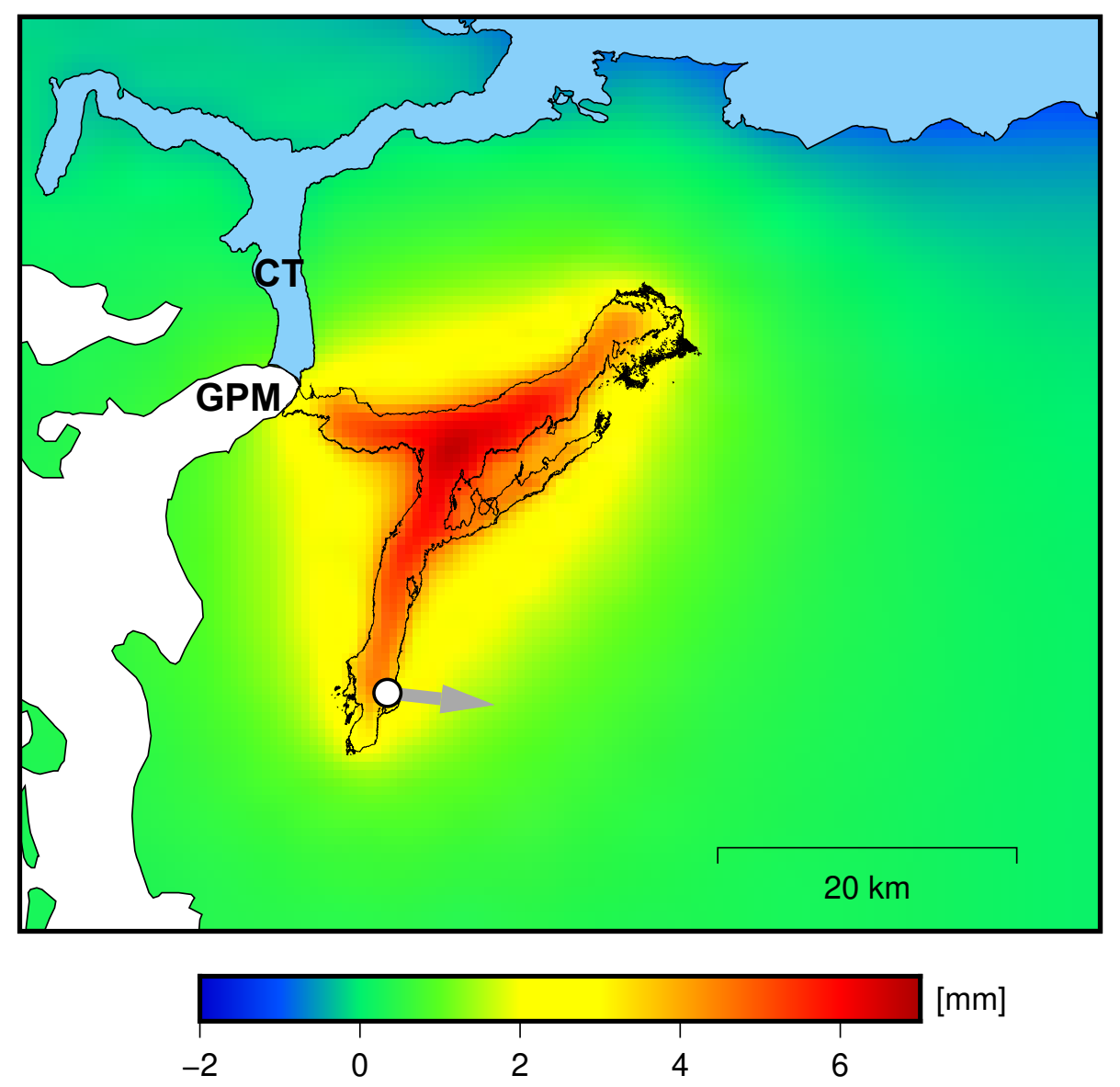

Figura 5.9: Efecto de carga de deformación vertical determinado con base en la aplicación del modelo de carga correspondiente al nivel máximo determinado para 2016 por endicamiento del Glaciar Perito Moreno. Se muestra la ubicación del punto de observación y se representa el vector de desplazamiento horizontal determinado en el procesamiento. El contorno negro indica la línea de costa para el nivel correspondiente, según tabla 4.2.

En la figura 5.9 se observa el resultado de los efectos de carga para el modelado correspondiente al máximo del año 2016. Durante la ruptura y descarga entre el 8 y el 12 de marzo, el nivel del los Brazo Rico y Sur cayó 7,04 $\mathrm{m}$ segun se indica en el capítulo 4. Al mismo tiempo, el nivel del lago Argentino se elevó en unos 0,68 m (BDHI, 2017). Observaciones GNSS continuas fueron registradas sobre roca en la costa Sureste 
del Brazo Sur (punto 3; ver figuras 5.1 y 5.12). Los datos GNSS fueron procesados de manera diferencial con Software Bernese GNSS 5.2 (Dach et al., 2015) y se obtuvieron posiciones diarias para las tres coordenadas. El desplazamiento del punto se deriva de las coordenadas medias antes (20 días) y después (27 días) de la descarga del embalse. A su vez correcciones por efectos de carga debieron aplicarse sobre los 27 días anteriores al rompimiento para determinar la deformación por aumento del nivel del agua para los días mencionados.

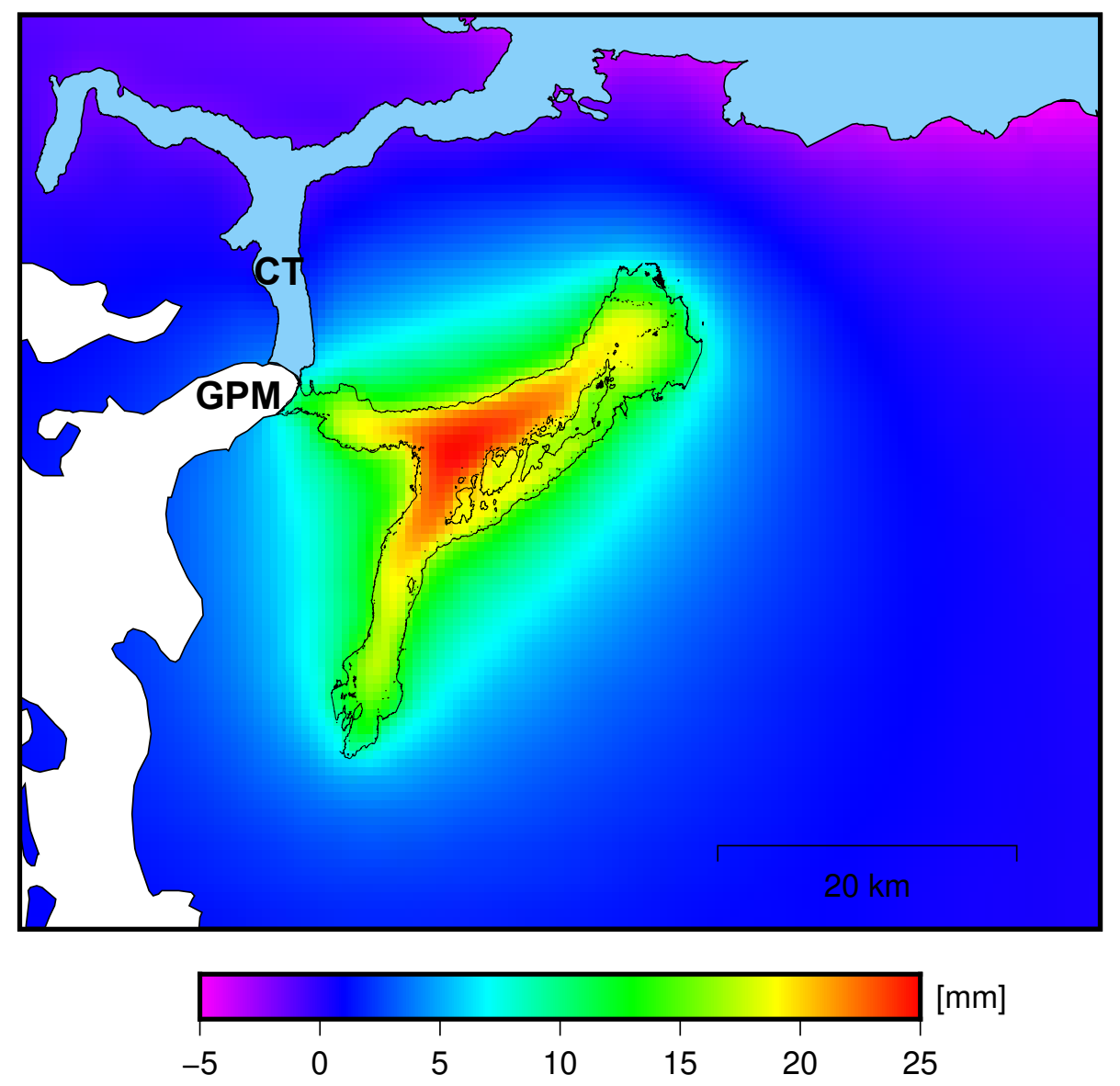

Figura 5.10: Efecto de carga para el modelo de carga correspondiente al máximo histórico registrado en el año 1956 de 24,5 metros (Guerrido et al., 2014). El contorno negro indica la línea de costa para el nivel correspondiente, según tabla 4.2.

Se presentan los resultados arrojados por el procesamiento (ver tabla 5.4). De estos se encuentra consistencia entre la deformación vertical observada y la modelada. Pero para el evento estudiado, estos resultados con una pequeña magnitud de la carga y por consiguiente deformación resultante, así también como el alto nivel de ruido en las observaciones, no permiten obtener una conclusión robusta sobre la validez regional del modelo de Tierra elástico aplicado. De la comparación entre los valores obtenidos se puede determinar que existe un acuerdo cualitativo entre el modelo y la observación. Por un lado parece haber un muy buen acuerdo en la componente vertical, mientras que en la horizontal se aprecia el sentido de la deformación (Marderwald et al., 2018a). 
Tabla 5.4: Comparación cualitativa entre los desplazamientos observados en la estación ubicada en la margen este del Brazo Sur y el modelado para el evento de 2016.

\begin{tabular}{lrr}
\hline Componente & $\begin{array}{r}\text { Observado } \\
{[\mathrm{mm}]}\end{array}$ & $\begin{array}{r}\text { Modelado } \\
{[\mathrm{mm}]}\end{array}$ \\
\hline Vertical & $3,6 \pm 2,5$ & 3,59 \\
Horizontal-N & $-0,2 \pm 1$ & $-0,61$ \\
Horizontal-E & $1.8 \pm 2,1$ & 0.25 \\
\hline
\end{tabular}

El glaciar Perito Moreno se ha embalsado en eventos anteriores con niveles de agua mayores a $20 \mathrm{~m}$ (Guerrido et al., 2014), por lo que, de futuras repeticiones de observación se podría obtener más información. Para ello, se determinan nuevos sitios de observación donde es posible esperar las mayores magnitudes de efectos de carga y por consiguiente dar mayor robustez al estudio. Los futuros sitios se establecen en función del modelado con la carga máxima registrada y determinando distintos niveles de altura de agua para determinar valores esperados. El figura 5.10 muestra los efectos de carga para el valor histórico máximo determinado.

Sobre la base de estos modelados se determinaron diferentes efectos, entre ellos, la inclinación (ver figura 5.11)de la superficie de la tierra solida relativo a una superficie equipotencial deformada. Sobre la margen norte del Brazo Rico se encuentran los valores máximo de este efecto. La magnitud supera el nivel de ruido de clinómetros según tabla 5.1 .

En la figura 5.12 se establece un posible punto de observación y se incluyen puntos cercanos dedicados al estudio del ajuste glacio-isostático y el efecto sobre estos. Los puntos graficados corresponden a un aumento del nivel de agua con pasos de $1 \mathrm{~m}$. El punto X indica un lugar óptimo para observaciones GNSS dedicado al estudio de efectos de carga por endicamiento de los Brazos Rico y Sur. La línea de costa es la determinada acorde al endicamiento máximo como se muestra en el modelo de carga en el capítulo 4. 


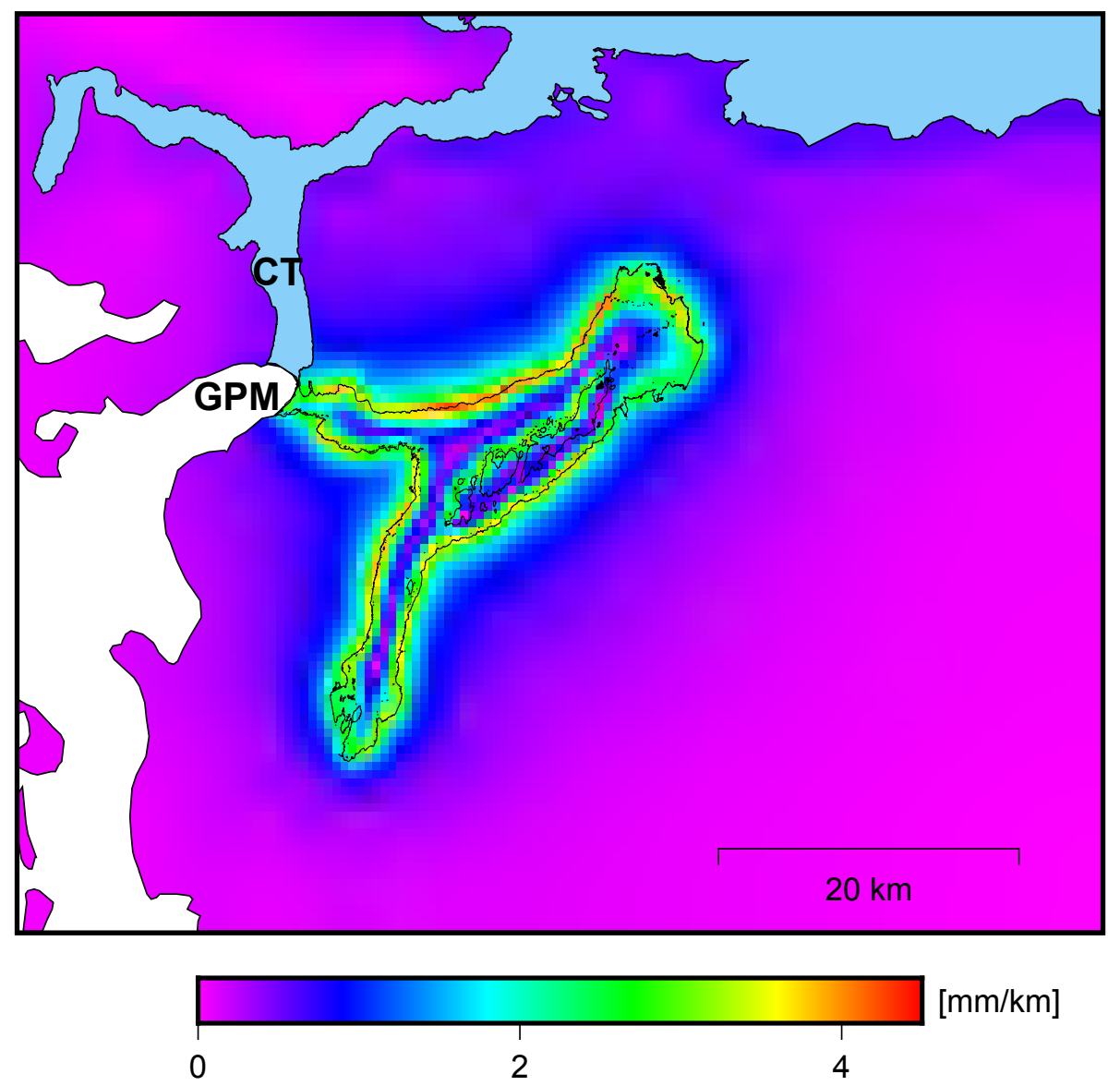

Figura 5.11: Modelo de inclinaciones correspondiente al máximo del 1956. Estas se determinaron del cálculo de efectos de carga para el potencial y la deformación vertical. El contorno negro indica la línea de costa para el nivel correspondiente, según tabla 4.2.

\subsubsection{Represas del Río Santa Cruz}

Con cotas del nivel máximo de endicamiento de 176.5 y 114 msnm (Massabie et al., 2007) y mediante el uso del modelo aerofotogramétrico desarrollado por el Instituto Geográfico Nacional se determinaron los modelos de carga para las represas Cóndor Cliff y Barrancosa, obteniendo una carga total de 5,34 y 3,63 Gt, respectivamente. Actualmente, observaciones GNSS en base al modelado se han proyectado en la inmediación de las represas buscando generar una solida determinación de las coordenadas previas al llenado de los embalses. Las deformaciones obtenidas mediante el modelado de efectos de carga representa una deformación elástica de la litosfera y se considera un efecto regional. Efectos locales, como subsidencia del terreno u otros, no son considerados en este trabajo.

Partiendo de efectos de carga de potencial y deformación vertical se determinó el cambio de altura ortométrica. En la figura 5.14 se puede apreciar en cercanías a la costa del embalse predicciones de cambios de altura ortométrica del orden del centímetro para varios puntos de la red de nivelación nacional de alta precisión. Estos cambios modelados de las alturas ortométrica superan en muchos de los puntos el nivel de ruido de observación de 


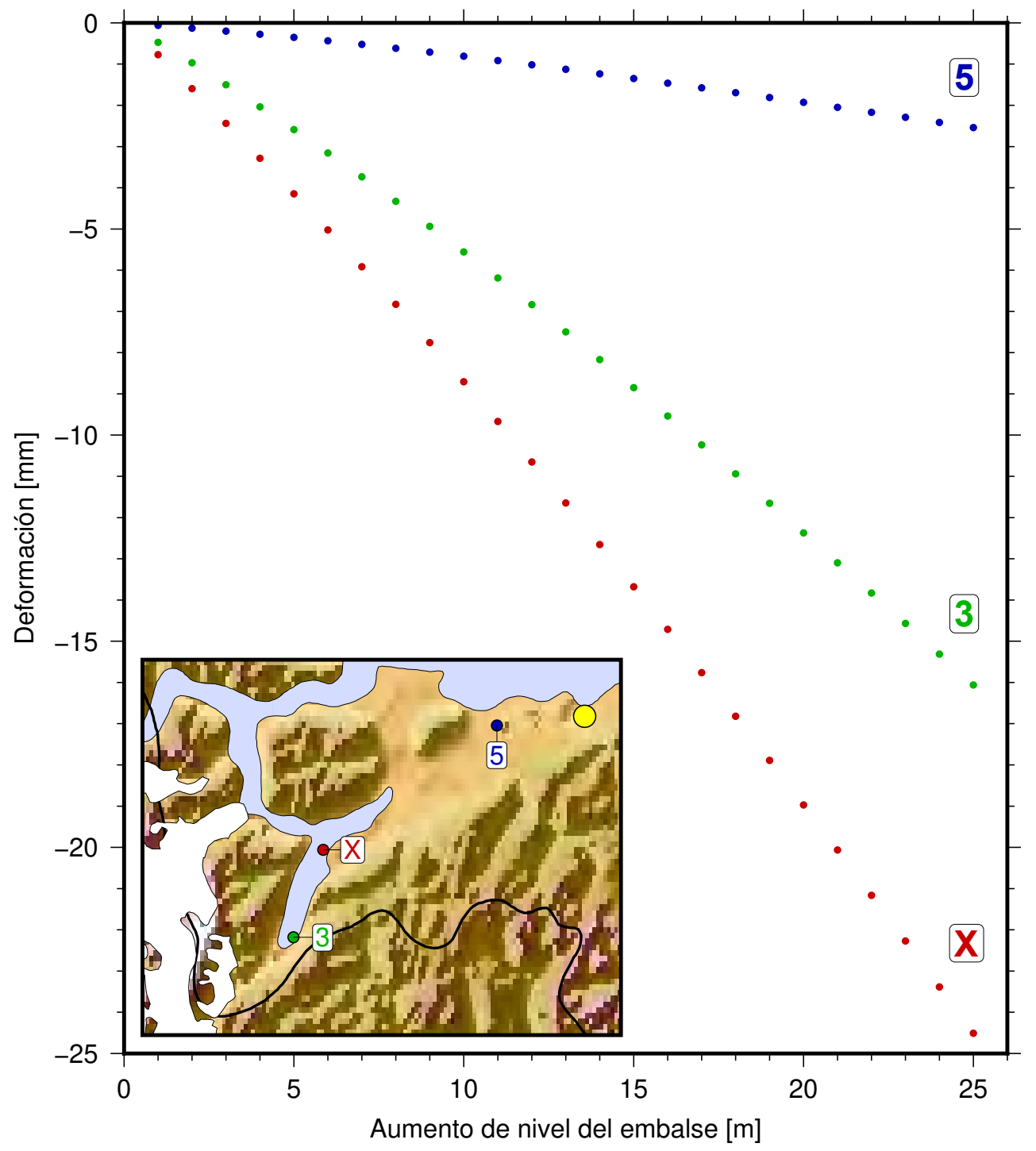

Figura 5.12: Curvas modeladas correspondiente a los efectos de carga para la deformación vertical correspondiente a pasos de un metro en el cambio de nivel de los Brazos Rico y Sur para tres sitios de observación. Puntos de observación 3 y 5, los correspondientes dedicados al estudio del ajuste glacio-isostático (ver figura 5.1).

la técnica. Esto pone en evidencia el impacto de la obra sobre la infraestructura geodésica oficial, no solo se enfrentará una perdida de varios puntos de nivelación como consecuencia de la inundación del embalse, sino también varios puntos sufren cambios significativos en sus valores publicados (altura ortométrica, gravedad). Por otro lado se concluye, que también este efecto puede ser observado por mediciones geodésicas de alta precisión, por esto motivo, puntos de observación pueden ser cuidadosamente seleccionados con el fin de proveer información sobre la respuesta de la tierra solida a cargas superficiales.

El modelo de gravedad para la región de las represas presenta magnitudes por encima del ruido de observaciones gravimétricas, para gravímetros absolutos como el FG5, según tabla 5.1. Sobre los puntos indicados se muestra un punto nodal de la Red Gravimétrica 
de Primer Orden (RPO-Ar). A diferencia de la precisión buscada para la mayoría de las observaciones, la determinación de estos puntos nodales se encuentra determinada con desvíos superiores a los $10 \mu$ gal (Instituto Geográfico Nacional de la República Argentina, 2016). 


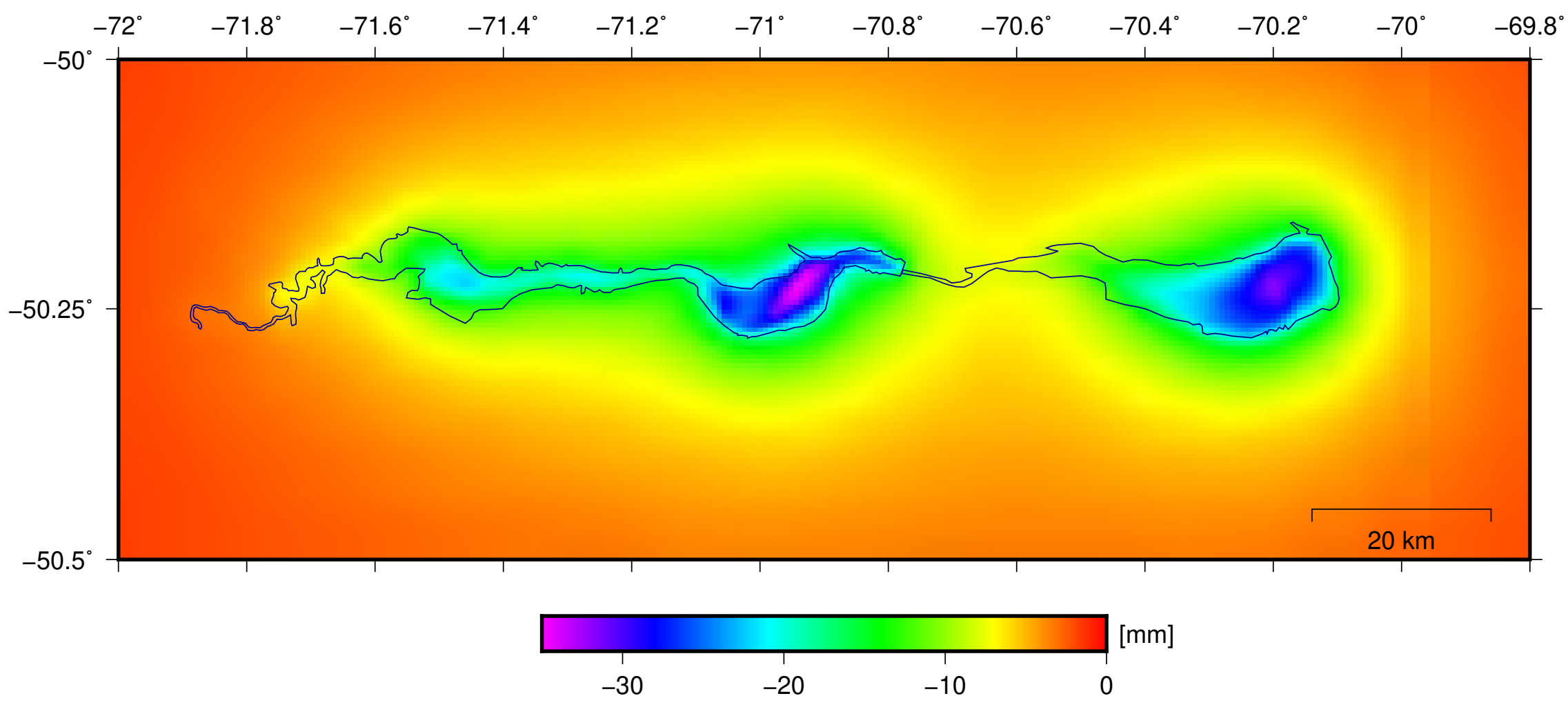

Figura 5.13: Modelo de deformación vertical correspondiente a las represas Cóndor Cliff y Barrancosa. El contorno azul representa la línea de costa de los embalses. 


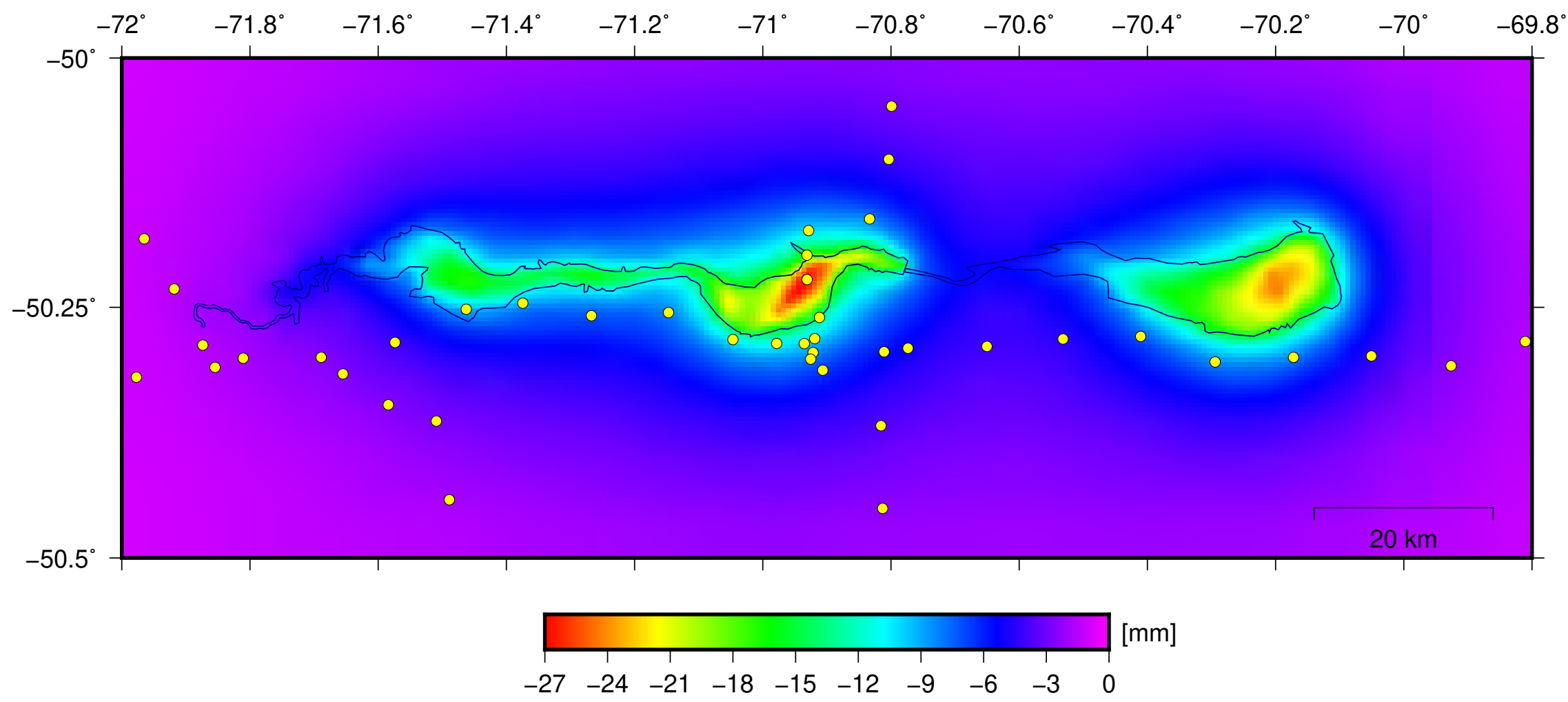

Figura 5.14: Cambio altura ortométrica en las represas Cóndor Cliff y Barrancosa. Los puntos amarillos corresponden a la Red de Nivelación de alta precisión (RN-Ar) (Instituto Geográfico Nacional de la República Argentina, 2016) 


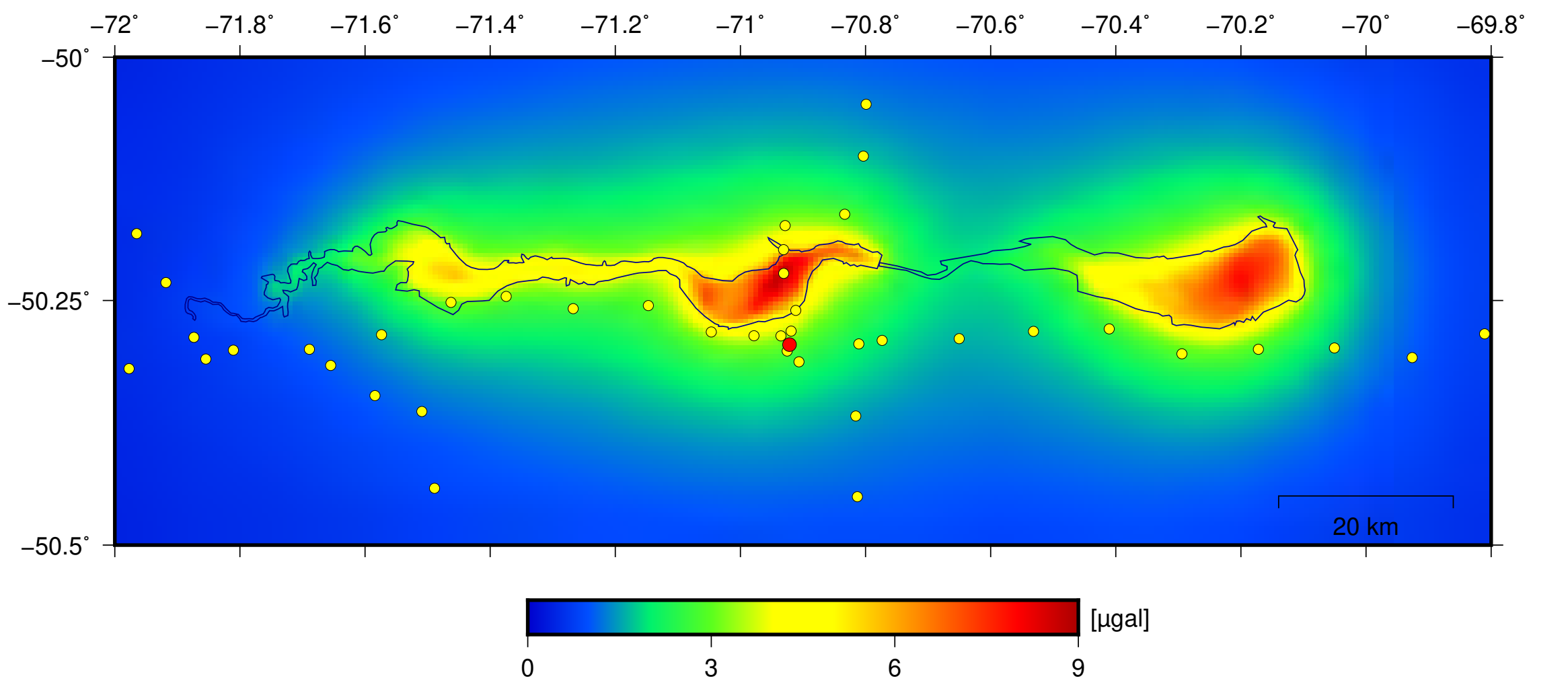

Figura 5.15: Cambio gravedad en las represas Cóndor Cliff y Barrancosa. Los puntos amarillos corresponden a la Red de Nivelación de alta precisión (RN-Ar). El punto rojo a la Red Gravimétrica de Primer Orden (RPO-Ar) (Instituto Geográfico Nacional de la República Argentina, 2016). 


\subsection{Efectos de carga glaciar}

La figura 5.16 muestra la deformación vertical elástica para el modelo de perdida de masa según Richter et al. (2019), donde, por un lado se observa la deformación debido a la tendencia anual por la perdida de masa de hielo neta correspondiente a 24,4 Gt (barra de colores inferior), y por otro lado, el cambio de posición máxima que experimenta cada punto en la coordenada vertical por efecto de la variación anual de la masa de hielo, correspondiente a una amplitud de 55 Gt (Richter et al., 2019).

El modelo presenta consistencia con modelados previos publicados para el efecto elástico en el Campo de Hielo Patagónico Sur (Lange et al., 2014). Por primera vez se presenta un modelo de respuesta elástica a la perdida de masas de hielo en el Campo de Hielo Patagónico Norte, donde hasta el momento su contribución elástica al ajuste glacioisostático se encontraba desestimada (Dietrich et al., 2010b). Determinaciones actuales de perdida de masa glaciar sugieren un mayor aporte por unidad de área sobre el Campo de Hielo Patagónico Norte (ver sección 4.4)(Richter et al., 2019). Esto queda representado por una mayor magnitud en la deformación que se concentra hacia este sector. Una estimación de la respuesta elástica a variaciones de masa glaciar requeriría observaciones continuas en puntos estratégicos. Esto es objetivo de nuevos proyectos en la región con el fin de mejorar la determinación del ajuste glacio-isostático, de manera de entender y establecer una mejor relación entre la perdida de masa de hielo en respuesta al cambio climático y la estructura mecánica de la Tierra. 


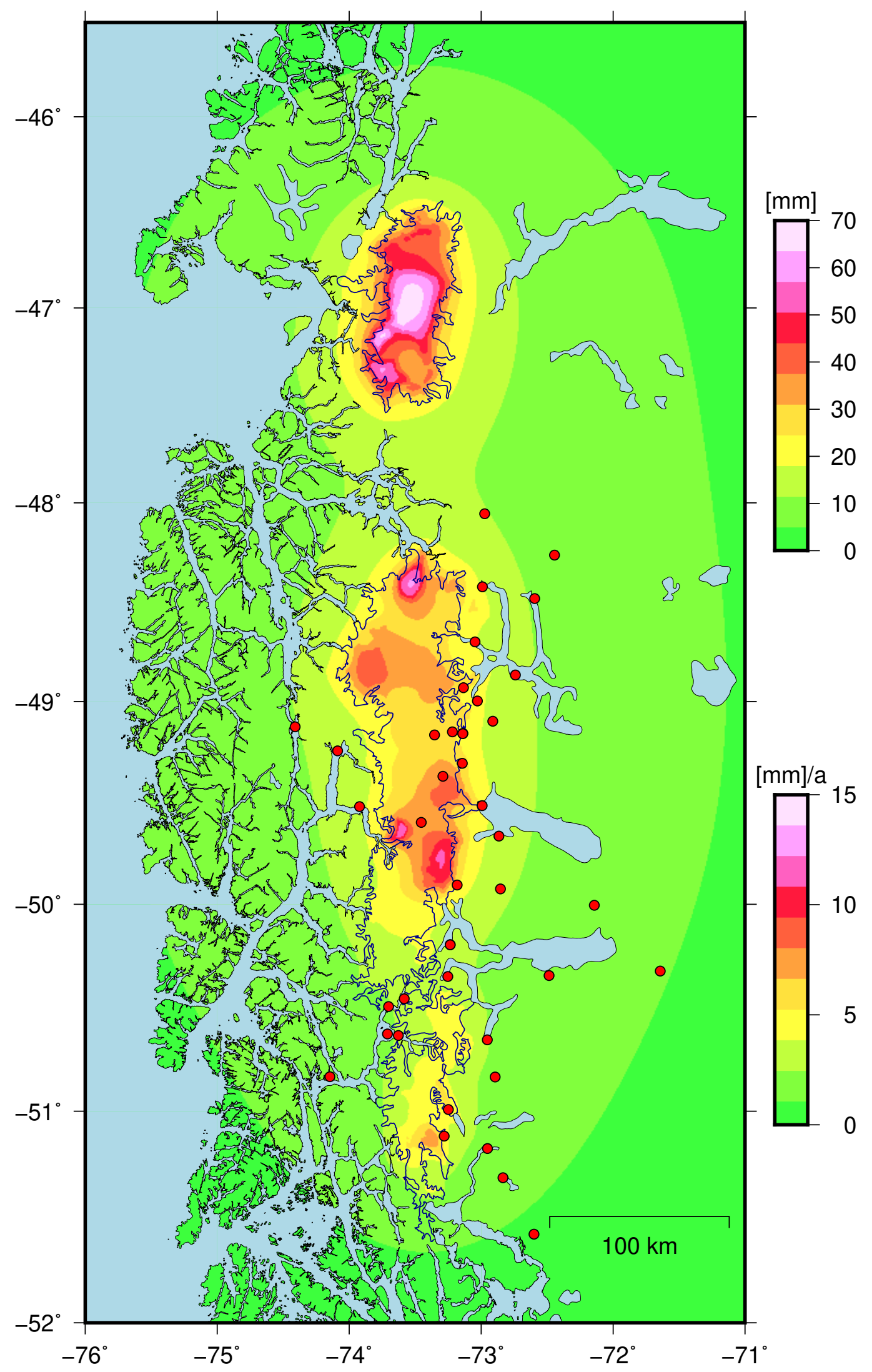

Figura 5.16: Campos de Hielo Patagónico Norte y Sur, deformación vertical elástica. La escala de colores superior representa el cambio en la vertical de las coordenadas de los puntos debido a la variación anual de las masas de hielo correspondientes a una amplitud de 55 Gt. La escala inferior representa el levantamiento debido a la perdida de masa neta anual de 24,4 Gt/a. 


\section{Capítulo 6}

\section{Integración y aplicaciones}

Para entender el sistema terrestre y sus cambios temporales, es necesaria una descripción precisa de los procesos geofísicos que implican el transporte de masa. A escala global, el ciclo hidrológico integra los procesos más relevantes asociados con intercambio de masas entre océanos, el continente y la atmósfera para escalas de tiempo estacionales e interanuales. Asimismo, redistribución de las masas en la atmósfera, caracterizados por cambios en la presión, el balance de masas en los círculos polares asociados a nivel eustático del mar y su influencia en las corrientes marinas desempeñan un rol importante. Estos procesos son simulados por modelos de circulación oceánica, hidrológicos, atmosféricos y cambio en las masas de hielo, respectivamente. Variaciones temporales de las masas en la criósfera, hidrosfera continental, atmósfera y océanos llevan a cambios en el potencial del campo gravitacional asociado a deformaciones de la corteza terrestre por efectos de carga. Para evaluar la validez y la incertidumbre de estos modelos globales, se requieren mediciones independientes del transporte de masas o de los cambios temporales de masa resultantes. Mientras variaciones del campo de gravedad a escala global son observadas por GRACE-FO, anteriormente GRACE, técnicas espaciales como GNSS, Very Long Base Interferometry (VLBI), Satellite Laser Ranging (SLR) y otras, permiten la medición de los desplazamientos de la posición del sitio. En particular, mediciones gravimétricas y geométricas se complementan unas a otras (Davis et al., 2004; van Dam et al., 2007). Las mediciones GNSS se ven afectadas significativamente por los desplazamientos del sitio causados por la deformación de la corteza. La aplicación de series temporales de deformaciones relacionadas con la reducción de la observación mediante GNSS no es todavía una norma común. Más bien, el efecto de los desplazamientos residuales se mantiene en las observaciones ajustadas y hace que las estimaciones de las coordenadas del emplazamiento muestren variaciones sistemáticas con respecto a una posición media (modelo lineal incluyendo las velocidades). Por ejemplo, Dach et al. (2011) mostraron una mejor repetitividad de las alturas semanales de las estaciones GNSS entre el 10 y el $20 \%$ en promedio si se aplican correcciones para el efecto de la carga de presión atmosférica a las observaciones GNSS (Fritsche et al., 2012). 


\subsection{Integración espectral}

Gran parte de los procesos que causan variaciones y/o transporte de masas en la superficie terrestre responden a cambios estacionales con periodos bien definidos como son las variaciones del nivel de los lagos, tasas de acumulación y pérdida de masas de hielo. Estos pueden ser representados gráficamente en espectros de amplitud, con máximos de amplitud en sus periodos de recurrencia. Sobre la base de datos de mareógrafos en grandes lagos (Base de datos Hidrología Integrada, (BDHI, 2017)) se obtienen las componentes anual y semia-anual más importantes de variación de nivel de agua. Datos de GRACE proveen información de cambios de masa de hielo. Datos sinópticos de estaciones meteorológicas proveen información sobre cambios de presión y cambios estacionales en las precipitaciones (siendo de gran relevancia en lugares como la Amazonía según se muestran los modelos hidrológicos globales, (Sood and Smakhtin, 2015)). En cambio, otros procesos, como el endicamiento producido por el glaciar Perito Moreno, no tienen un periodo de recurrencia bien definido, pero como se ha mostrado, puede ser cuantificable e integrado a las observaciones. Otros, como transporte de sedimentos los cuales son de muy difícil separación debido a su complejidad en la cuantificación y tiempos de transporte y acumulación, además de requerir un modelado más complejo, así es también el caso del cambio del nivel eustático del mar. Sesiones de observaciones GNSS diarias (24 horas) permiten eliminar algunos de los efectos de mareas terrestres y mareas oceánicas en el caso de estaciones cercanas a la costa en frecuencias diarias y superiores, Pero así mismo, permiten evaluar el ajuste de los modelos regionales y/o globales de efectos de carga donde los fenómenos naturales se encuentran en las frecuencias mencionadas. Distinto es el caso para fenómenos no recurrentes como es el caso de embalses artificiales, pero que en ciertos casos pueden corresponder con condiciones climáticas estacionales o con periodos de años que pueden alterar el nivel de los mismos (por ejemplo, sequías). La observación de todos estos procesos permite la comparación con los modelados de efectos de carga, así se provee una herramienta de validación, mejora y ajuste tanto de modelos de Tierra como de carga destinados a la reducción de efectos de los mismos sobre las observaciones como al estudio de los mismos.

La figura 6.1 muestra un gráfico cualitativo de los principales efectos de carga presentes en Patagonia austral. A modo de ejemplificar y resumir esquemáticamente, se exhiben las principales componentes, para las cuatro frecuencias en las que se ha hecho énfasis en este trabajo y se mencionan en el gráfico. Con componentes de marea oceánica semi-diaria (M2, componente principal de marea) como más significativa en el efecto de carga oceánica, seguido de las componentes diurnas como K1 D'onofrio et al. (2016). Contrariamente, aportes por efectos de carga atmosférica se consideran prácticamente despreciables, estando estos representados por una componente de muy pequeña magnitud o encontrarse limitados a periodos muy cortos y de poca extensión para sus amplitudes máximas (ver figura 5.5). En contraste, una gran importancia adquieren las componentes anual y semianual correspondiente a los efectos de carga aportados por la variabilidad de las masas en los Campos de Hielo Patagónico, cuyo interés radica en su utilidad como indicadores de cambio climático. Por otro lado, la carga hidrológica debida a la variación de nivel de agua de los lagos, sujeta a los cambios estacionales en los campos de hielo, presenta 


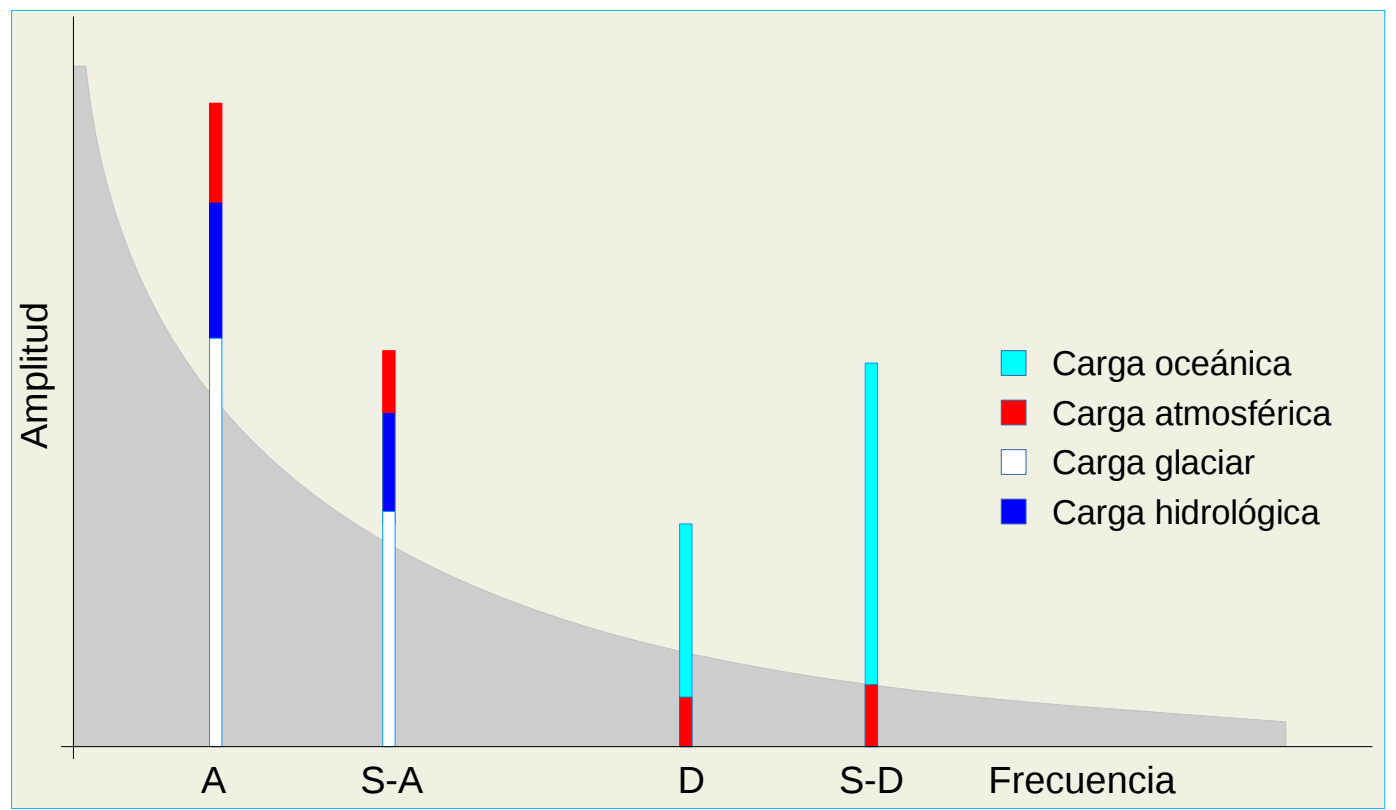

Figura 6.1: Dibujo esquemático de espectro de amplitudes para las variaciones anual (A), semi-anual (S-A), diaria (D) y semi-diaria (S-D). Se representa las cuatro componentes estudiadas, carga oceánica, atmosférica, hidrológica y glaciar, para los periodos mencionados en Patagonia austral. El gráfico se encuentra fuera de escala.

una mayor magnitud en su componente anual; esta ha de ser considerada en mediciones llevadas a cabo sobre sitios muy cercanos a la costa de los lagos, donde se encuentra la mayor concentración de las masas (ver figura 5.7). De esta manera, con un espectro de amplitud cualitativo es posible ejemplificar como se integran en sus respectivas frecuencias los efectos de carga para las variaciones de masas de diverso origen.

\subsection{Integración local}

Se determinaron series temporales en dos puntos (figura 6.2). En ambas, se integran las componentes por efecto de carga glaciar e hidrológica anual y semi-anual. Se observa con claridad las diferencias en ambas series temporales, en la tendencia y amplitudes de las variaciones de la coordenada vertical para ambos puntos ubicados a distintas distancias del campo de hielo. La serie correspondiente al punto próximo al glaciar Upsala (punto 10) tiene una componente muy marcada por los cambios estacionales de las masas de hielo así como función de la tendencia anual en la perdida de masa. Pese a estar ubicado próximo a uno de los brazos del Lago Argentino, el efecto de carga hidrológica no llega a ser considerable debido a que la variación en la masa de agua no es cuantiosa frente al efecto de carga glaciar sobre el área circundante. De la misma manera, la mayor distancia al campo de hielo disminuye los efectos de la carga glaciar. Sobre el punto cercano a la localidad de El Calafate (punto 5), se aprecia una componente del efecto de carga hidrológica de consideración, al ver las diferencias entre las dos curvas. Los efectos de 
carga dependen de la masa y la distancia a la misma. La morfología del lago en este sector permite concentrar una mayor masa en un área cercana al punto, lo que produce efectos de carga de mayor magnitud. Otro componente importante a notar es el cambio de las velocidades en la coordenada vertical, quedando evidenciada por el cambio de pendientes para ambas curvas, relativo no solo a la tendencia, sino también a la cercanía a la carga y máximo en la deformación.

Es importante de recordar que los efectos de carga modelados se desarrollaron sobre un modelo de Tierra perfectamente elástico. Por lo tanto, para los modelos de carga que involucran una variación temporal de las masas que se encuentran en el espectro de bajas frecuencias, es de esperar que una mejora se obtenga con un modelado visco-elástico. Esta clase de modelado presenta mayores dificultades en su determinación, dado que involucra un cálculo más complejo y donde intervienen mayor cantidad de variables, como la viscosidad del manto superior y el tiempo para los modelos de carga; siendo también más difícil alcanzar una alta resolución en el modelado. Mayormente, la respuesta cercana a la carga se rige por la estructura del manto superior y la corteza. Ésta respuesta afecta fuertemente sólo a los modos de alto grado de la Tierra (Farrell, 1972). Por ésta razón, para los modelos de carga presentes en este trabajo, consideramos al modelado de efectos de carga elástico como una buena primera aproximación y valido para el campo cercano.

Actualmente, la gran mayoría de los estudios llevados a cabo han hecho hincapié en la determinación del balance de masas anual, con diversas estimaciones realizadas mediante sensores remotos (Braun et al., 2019; Foresta et al., 2018; Malz et al., 2018; Reager et al., 2016; Willis et al., 2012a,b). Observaciones continuas en puntos previamente determinados permitiría establecer una mejor correlación entre la deformación por efectos de carga y la determinación en el balance de masa anual y así también poder separar la componente viscoelástica. La posibilidad de obtener información de la deformación correspondiente a la componente viscoelástica permitiría ajustar mejor los modelos de perdida de masa glaciar desarrollados hasta la pequeña edad de hielo (Lange et al., 2014) y por consiguiente lograr un aporte al estudio del cambio climático. 


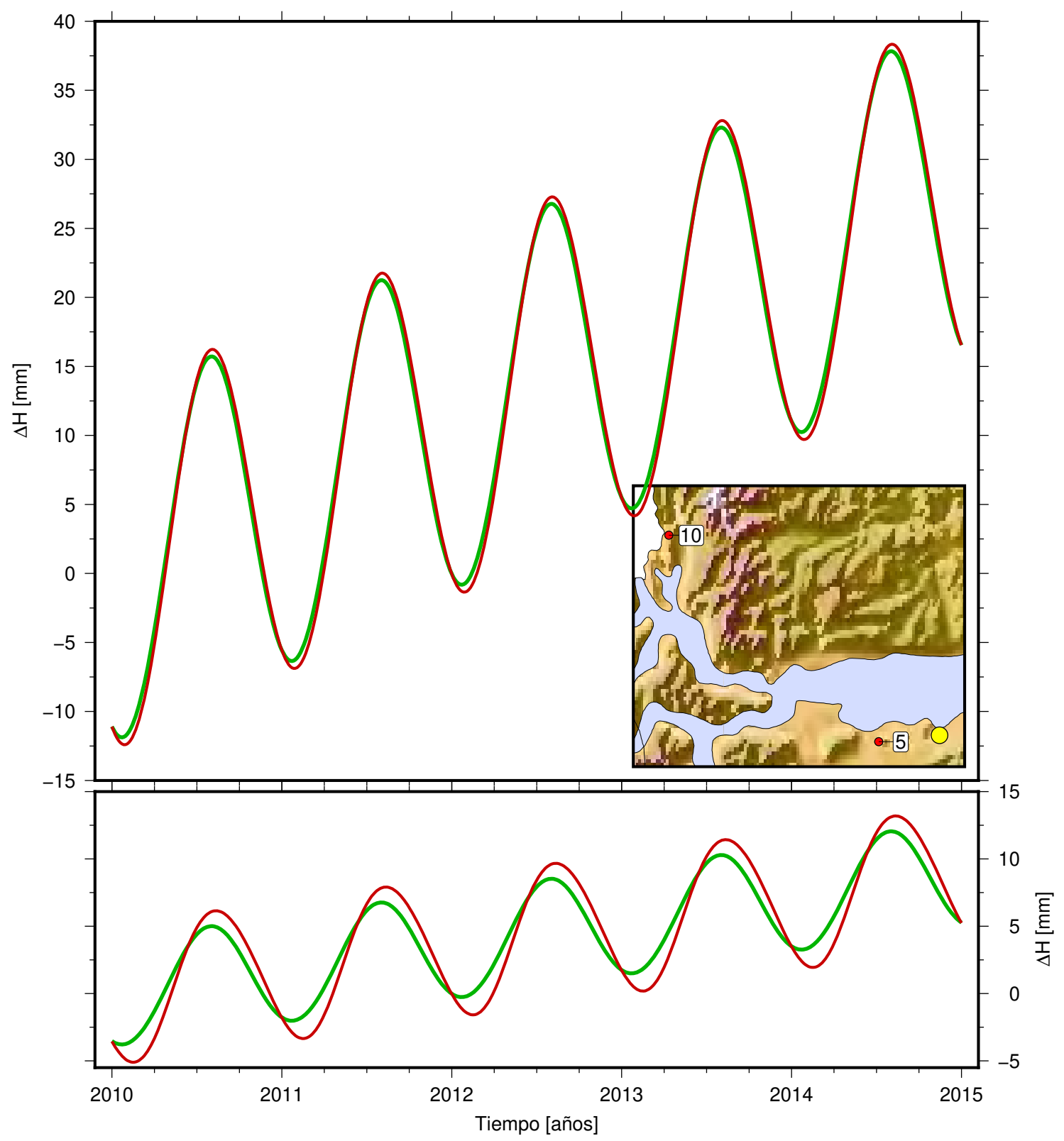

Figura 6.2: Series temporales correspondiente a los modelos de variación de la coordenada vertical por efectos de carga debido a cambios de masa, anual y semi-anual en los campos de hielo patagónico y los grandes lagos (curvas rojas). Estas corresponden a los puntos 10 (gráfico superior) y 5 (gráfico inferior) (ver figura 5.1). Las curvas verdes indican el cambio de la coordenada vertical, debido únicamente, por efecto de la variación de masa glaciar. El punto amarillo indica la localidad de El Calafate. Ambos gráficos se encuentran a la misma escala en las ordenadas. 


\section{Capítulo 7}

\section{Conclusiones}

De los resultados obtenidos para los modelos de carga desarrollados, se estiman magnitudes geodésicamente observables para la respuesta elástica de la Tierra sólida a problemas glaciológicos e hidrológicos a cargas de corto plazo. Recientes observaciones sismológicas llevadas a cabo en sucesivas campañas (Wiens et al., 2017), permitirán establecer y mejorar nuevos modelos de Tierra para la región. La observación de los efectos de la carga elástica está en curso. Esto permitirá una validación o mejora regional de los modelos.

Se muestran por primera vez modelados de la respuesta elástica con base en modelos de variación anual de las masas de hielo para los Campos de Hielo Patagónico Norte y Sur, conjuntamente con los efectos de carga hidrológicos. Los efectos de carga de corto plazo degradan la determinación de las tasas de elevación debido a ajuste glacio-isostático observadas por GNSS. Las contribuciones viscoelásticas a las tasas de levantamiento debido a los cambios en el espesor del hielo en el pasado, tienden a ser varias veces las tasas elásticas. Los patrones espaciales de las contribuciones elásticas y viscoelásticas no tienen por qué ser similares. Estas últimas pueden ser eliminadas del problema de la combinación lineal entre gravedad observada y levantamiento obtenido con técnicas geodésico satelital. Los resultados del modelado permiten corregir estos efectos. Resultados que contribuyen a mejorar la cuantificación de las tasas de pérdida de masa de hielo actuales, validar y/o mejorar nuevos modelos de Tierra regionales y establecer nuevos sitios de observación destinados a observación de efectos de carga elásticos y permitir la separación de tasas de elevación debido a ajuste glacio-isostático.

Desarrollos de modelos independientes, como resulta ser el caso de las represas permiten cuantificar su impacto en la región sobre puntos GNSS destinados a la observación de la deformación glacio-isostática, y asimismo, sobre puntos cercanos de la red de nivelación oficial. Al mismo tiempo se plantea la observación local a los efectos de carga de las represas como una oportunidad única en la validación de modelos de Tierra para la región. Tal validación tendrá implicancias directas sobre el modelado de los efectos de carga generados por los cambios actuales de la masa glaciar y su separación de la respuesta visco-elástica a cambios de masa de hielo en el pasado.

Este trabajo además de producir estimaciones por efectos de carga en el sur de 
la Patagonia, generó una herramienta de cálculo de estos efectos aplicable a modelos independientes, tanto de Tierra como de carga siguiendo la metodología desarrollada por Farrell (1972), la cual puede ser aplicable a cualquier otra región. Programa que puede ser utilizado para la aplicación de correcciones a serie de datos, cuyas observaciones geodésicas se encuentran afectadas por efectos de carga y requieran ser eliminados, o para contrastar entre observaciones y diferentes modelos.

El estudio de efectos de carga elástica plantean nuevos interrogantes y permiten evaluar distintas perspectivas a futuro. Se plantean nuevas preguntas en la determinación de pérdida de masa glaciar, sus variaciones y patrón espacial, como por ejemplo, ¿Cómo establecer una mejor correlación entre la pérdida de masa y la deformación en el tiempo? ¿Hasta dónde es factible usar modelos puramente elásticos en la región? ¿Cuál es el aporte de la tectónica regional en distintos intervalos temporales?.

Se evalúan diferentes puntos de vista a futuro. Mejoras del código para efectos de carga, esto comprende la incorporación y validación de nuevos efectos, como determinación de inclinaciones y cálculos vectoriales de mejor ajuste a la posición de la carga y punto de cálculo, estableciendo una mejor dependencia para la componente de gravedad. Recientemente se ha iniciado un proyecto de medición de gravedad absoluta en puntos estratégicos cercanos a la región del Campo de Hielo Patagónico Sur (Scheinert and Rulke, 2018) con la idea de determinar la respuesta de la Tierra solida a los cambios de masa de hielo. Esta clase de observaciones requieren para su interpretación incluir los efectos de carga elástico en respuesta a variaciones de masa de cortos periodos, tanto hidrológicos como glaciológicos. Esto contribuirá a mejorar la cuantificación del ajuste glacio-isostático en la región del Campo de Hielo Patagónico Sur, estudio que no pueden ser fácilmente determinados por solo una técnica de observación (hasta el momento solo GNSS). Mejorar e incorporar modelos de carga glaciar con dependencia temporal para el modelado visco-elástico es una parte fundamental para imponer condicionamientos a modelos de Tierra a respuestas por efectos de carga, evaluando modelos de Tierra mas complejos. El desarrollo de modelos glaciológicos requiere la evaluación de diferentes criterios en la determinación del retroceso glaciar en el pasado, siendo los espesores de hielo una variable difícil de determinar debido que presenta una gran incertidumbre. Observaciones y análisis de formaciones geomorfológicas influenciadas por la tectónica regional podían contribuir al conocimiento geodinámico en la Patagonia austral, como es el caso de desniveles entre paleo-terrazas lacustres del Lago Argentino (Marderwald et al., 2018b). 


\section{Índice de figuras}

1.1. Patagonia austral . . . . . . . . . . . . . . . . . . . . . 2

3.1. Patagonia austral, limite de placas y topografía . . . . . . . . . . 20

4.1. Espectro amplitudes de variación de presión atmosférica . . . . . . . . . 28

4.2. Ubicación de estaciones sinópticas . . . . . . . . . . . . . . . . . . . . 30

4.3. Variación anual de nivel de los grandes lagos . . . . . . . . . . . . . . . 31

4.4. Modelo de carga correspondiente al endicamiento del glaciar Perito Moreno 34

4.5. Modelos de carga de las represas Cóndor Cliff y La Barrancosa . . . . . . . 36

4.6. Modelos de carga glaciar . . . . . . . . . . . . . . . . . . . . . 39

5.2. Efecto de carga oceánica, componente M2 f . . . . . . . . . . . . 46

5.3. Diferencias entre los modelos EOT11a y D'onofrio et al. (2016) . . . . . . . 47

5.4. Efecto de carga oceánica del modelo regionalizado para la componente M2 48

5.5. Carga atmosférica y efectos . . . . . . . . . . . . . . . 50

5.6. Evento atmosférico . . . . . . . . . . . . . . . . . 51

5.7. Efecto de carga para los grandes lagos - Componente vertical . . . . . . . . 53

5.8. Efecto de carga para los grandes lagos - Gravedad . . . . . . . . . . . . 54

5.9. Efecto de carga para Brazo Rico y Brazo Sur, sobre modelo del 2016 . . . . 55

5.10. Efecto de carga para Brazo Rico y Brazo Sur, sobre modelo del 1956 . . . . 56

5.11. Efecto de carga para Brazo Rico y Brazo Sur, inclinaciones, sobre modelo del $1956 \ldots \ldots \ldots \ldots$. . . . . . . . . . . . . . . . . . 58

5.12. Curvas de deformación para 3 sitios de observación . . . . . . . . . . . 59

5.13. Modelo de deformación vertical correspondiente a las represas Cóndor Cliff y Barrancosa. . . . . . . . . . . . . . . . . . . 61

5.14. Cambio altura ortométrica en las represas Cóndor Cliff y Barrancosa . . . 62

5.15. Efecto de carga gravimétrico en las represas Cóndor Cliff y Barrancosa . . 63

5.16. Campos de Hielo Patagónico Norte y Sur, deformación vertical elástica . 65

6.1. Espectro esquemático de amplitudes para Patagonia Austral . . . . . . . . 69 
6.2. Series temporales correspondiente a los modelos de variación de la coordenada vertical por efectos de carga . . . . . . . . . . . . . . . . . . 71 


\section{Índice de tablas}

4.1. Diferencias entre máximo y mínimo de variación anual de los lagos . . . . . 32

4.2. Niveles de agua determinados para los Brazos Rico/Sur . . . . . . . . . . . 33

5.1. Métodos de observación geodésicos . . . . . . . . . . . . . . . . . . . 42

5.2. Comparación componente semi-diurna M2 . . . . . . . . . . . . . . . . . 42

5.3. Comparación de los resultados obtenidos con ELOADE y Bos and Scherneck (2011) para la deformación vertical de las componentes semi-diurnas y diurnas más importantes. $\mathrm{R}$ es el promedio de los módulos de las diferencias relativas. Los valores se calcularon sobre los 44 puntos indicados en la figura 5.1. . . . . . . . . . . . . . . . . . 45

5.4. Comparación cualitativa entre observado y modelado . . . . . . . . . . . 57 


\section{Bibliografía}

ACT EIA PRESAS SC, R. P. (2017). Estudio de impacto ambiental aprovechamientos hidroeléctricos del río santa cruz (presidente dr. néstor c. kirchner y gobernador jorge cepernic), provincia de santa cruz. Online, accedido el 20 Septiembre 2018.

Agnew, D. and Wyatt, F. (2003). Long-base laser strainmeters: A review. scripps institution of oceanography technical report.

Agnew, D. C. (1986). Strainmeters and tiltmeters. Reviews of Geophysics, 24(3):579-624.

Agnew, D. C. (1997). Nloaf: A program for computing ocean-tide loading. Journal of Geophysical Research: Solid Earth, 102(B3):5109-5110.

ALOS-PALSAR (2017). Dataset: [https://www.asf.alaska.edu, 2015], [PALSAR Radiometric Terrain Corrected high res]; Includes Material @ JAXA/METI [2007]. Accessed through ASF DAAC, [https://www.asf.alaska.edu] [2017].

Altamimi, Z., Rebischung, P., Métivier, L., and Collilieux, X. (2016). Itrf2014: A new release of the international terrestrial reference frame modeling nonlinear station motions. Journal of Geophysical Research: Solid Earth, 121(8):6109-6131.

Alterman, Z., Jarosch, H., Pekeris, C. L., and Jeffreys, H. (1959). Oscillations of the earth. Proceedings of the Royal Society of London. Series A. Mathematical and Physical Sciences, 252(1268):80-95.

Amante, C. and Eakins, B. (2009). Etopo1 1 arc-minute global relief model: Procedures, data sources and analysis. NOAA Technical Memorandum NESDIS NGDC-24. accedido en 2018.

Backus, G. E. (1967). Converting Vector and Tensor Equations to Scalar Equations in Spherical Coordinates*. Geophysical Journal International, 13(1-3):71-101.

Baker, T. F. (1984). Tidal Deformations of the Earth. Science Progress, 69:197-233.

Baker, T. F. and Bos, M. S. (2003). Validating earth and ocean tide models using tidal gravity measurements. Geophysical Journal International, 152(2):468-485.

Barletta, V. R., Bevis, M., Smith, B. E., Wilson, T., Brown, A., Bordoni, A., Willis, M., Khan, S. A., Rovira-Navarro, M., Dalziel, I., Smalley, R., Kendrick, E., Konfal, S., Caccamise, D. J., Aster, R. C., Nyblade, A., and Wiens, D. A. (2018). Observed rapid bedrock uplift in amundsen sea embayment promotes ice-sheet stability. Science, 360(6395):1335-1339. 
BDHI (2017). Base de Datos Hidrológica Integrada, Ministerio de Obras Públicas. http://bdhi.hidricosargentina.gob.ar/.

Beltrán, A. (1997). Caracterización microclimática del distrito occidental de la estepa patagónica. Magister Thesis, University of Buenos Aires, AR.

Bird, P. (2003). An updated digital model of plate boundaries. Geochemistry, Geophysics, Geosystems, 4(3).

Bos, M. and Scherneck, H. (2011). Ocean tide loading provider. Retrieved December, $1: 2014$.

Bos, M. S., Penna, N. T., Baker, T. F., and Clarke, P. J. (2015). Ocean tide loading displacements in western europe: 2. gps-observed anelastic dispersion in the asthenosphere. Journal of Geophysical Research: Solid Earth, 120(9):6540-6557.

Braun, M. H., Malz, P., Sommer, C., Farías-Barahona, D., Sauter, T., Casassa, G., Soruco, A., Skvarca, P., and Seehaus, T. C. (2019). Constraining glacier elevation and mass changes in south america. Nature Climate Change, 9(2):130-136.

Breitsprecher, K. and Thorkelson, D. J. (2009). Neogene kinematic history of nazca-antarctic-phoenix slab windows beneath patagonia and the antarctic peninsula. Tectonophysics, 464(1):10 - 20. Interpreting the tectonic evolution of Pacific Rim margins using plate kinematics and slab window volcanism.

Carrere, L., Lyard, F., Cancet, M., Guillot, A., Picot, N., et al. (2016). Fes 2014, a new tidal model - validation results and perspectives for improvements. In Proceedings of the ESA living planet symposium, pages 9-13.

Casassa, G. and Rivera, A. (1999). Topographic mass balance model for the southern patagonia icefield. Annals of Glaciology.

Casassa, G., Rivera, A., Aniya, M., and Naruse, R. (2002). Current Knowledge of the Southern Patagonia Icefield, pages 67-83. Springer US, Boston, MA.

Casassa, G., Rodríguez, J. L., and Loriaux, T. (2014). A New Glacier Inventory for the Southern Patagonia Icefield and Areal Changes 1986-2000, pages 639-660. Springer Berlin Heidelberg, Berlin, Heidelberg.

Casassa, G., Sepúlveda, F. V., and Sinclair, R. M. (2012). The Patagonian icefields: a unique natural laboratory for environmental and climate change studies. Springer Science \& Business Media.

Clarke, A. J. and Battisti, D. S. (1981). The effect of continental shelves on tides. Deep Sea Research Part A. Oceanographic Research Papers, 28(7):665 - 682.

Comisso, E. (2017). Guardaparque. Seccional Río Mitre. Parque Nacional Los Glaciares.

Dach, R., Böhm, J., Lutz, S., Steigenberger, P., and Beutler, G. (2011). Evaluation of the impact of atmospheric pressure loading modeling on gnss data analysis. Journal of Geodesy, 85(2):75-91. 
Dach, R., Lutz, S., Walser, P., and Fridez, P. (2015). Bernese gnss software version 5.2. user manual, astronomical institute. Bern: University of Bern, Bern Open Publishing. https://doi. org/10.7892/boris, 72297.

Dahlen, F. A., Smith, M. L., and Batchelor, G. K. (1975). The influence of rotation on the free oscillations of the earth. Philosophical Transactions of the Royal Society of London. Series A, Mathematical and Physical Sciences, 279(1292):583-624.

Darwin, G. H. (1882). Xlvi. on variations in the vertical due to elasticity of the earth's surface. The London, Edinburgh, and Dublin Philosophical Magazine and Journal of Science, 14(90):409-427.

Davis, J. L., Elósegui, P., Mitrovica, J. X., and Tamisiea, M. E. (2004). Climate-driven deformation of the solid earth from grace and gps. Geophysical Research Letters, 31(24).

Dietrich, R., Ivins, E., Casassa, G., Lange, H., Wendt, J., and Fritsche, M. (2010a). Rapid crustal uplift in patagonia due to enhanced ice loss. Earth and Planetary Science Letters, $289(1): 22-29$.

Dietrich, R., Ivins, E., Casassa, G., Lange, H., Wendt, J., and Fritsche, M. (2010b). Rapid crustal uplift in patagonia due to enhanced ice loss. Earth and Planetary Science Letters, 289(1):22-29.

Dietrich, R., Rülke, A., and Scheinert, M. (2005). Present-day vertical crustal deformations in west greenland from repeated gps observations. Geophysical Journal International, 163(3):865-874.

D'onofrio, E., Oreiro, F., Grismeyer, W., and Fiore, M. (2016). Predicciones precisas de marea astronómica calculadas a partir de altimetría satelital y observaciones costeras para la zona de isla grande de tierra del fuego, islas de los estados y canal de beagle. GeoActa, 40:60-75.

Dussaillant, I., Berthier, E., Brun, F., Masiokas, M., Hugonnet, R., Favier, V., Rabatel, A., Pitte, P., and Ruiz, L. (2019). Two decades of glacier mass loss along the andes. Nature Geoscience, 12:802-808.

Dziewonski, A. M. and Anderson, D. L. (1981). Preliminary reference earth model. Physics of the Earth and Planetary Interiors, 25(4):297 - 356.

F., G. N., Harrison, S., N., J. K., K., A., and A., C. (2011). Global sea-level contribution from the patagonian icefields since the little ice age maximum. Nature Geoscience, 4:303-307.

Farrell, W. E. (1972). Deformation of the Earth by surface loads. Review of Geophysics and Space Physics, 10(3):761-797.

Flint, R. F. and Fidalgo, F. (1964). Glacial Geology of the East Flank of the Argentine Andes between Latitude $39^{\circ} 10^{\prime} \mathrm{S}$ and Latitude $41^{\circ} 20^{\prime} \mathrm{S}$. Geological Society of America Bulletin, 75(4):335-352. 
Foresta, L., Gourmelen, N., Weissgerber, F., Nienow, P., Williams, J., Shepherd, A., Drinkwater, M., and Plummer, S. (2018). Heterogeneous and rapid ice loss over the patagonian ice fields revealed by cryosat-2 swath radar altimetry. Remote Sensing of Environment, 211:441 - 455.

Francis, O. and Dehant, V. (1987). Recomputation of the green's functions for tidal loading estimations. Marées Terrestres Bulletin d'Informations, 100:6962-6986.

Francis, O. and Mazzega, P. (1990). Global charts of ocean tide loading effects. Journal of Geophysical Research: Oceans, 95(C7):11411-11424.

Fritsche, M., Döll, P., and Dietrich, R. (2012). Global-scale validation of model-based load deformation of the earth's crust from continental watermass and atmospheric pressure variations using gps. Journal of Geodynamics, 59-60:133 - 142. Mass Transport and Mass Distribution in the System Earth.

Gabriel, A. K., Goldstein, R. M., and Zebker, H. A. (1989). Mapping small elevation changes over large areas: Differential radar interferometry. Journal of Geophysical Research: Solid Earth, 94(B7):9183-9191.

Glorioso, P. D. and Flather, R. A. (1997). The patagonian shelf tides. Progress in Oceanography, 40(1):263 - 283. Tidal Science In Honour of David E. Cartwright.

Goad, C. C. (1980). Gravimetric tidal loading computed from integrated green's functions. Journal of Geophysical Research: Solid Earth, 85(B5):2679-2683.

Gorring, M. L. and Kay, S. M. (2001). Mantle processes and sources of neogene slab window magmas from southern patagonia, argentina. Journal of Petrology, 42(6):10671094.

Guerrido, C. M., Villalba, R., and Rojas, F. (2014). Documentary and tree-ring evidence for a long-term interval without ice impoundments from glaciar perito moreno, patagonia, argentina. The Holocene, 24(12):1686-1693.

Heinsheimer, G. (1959). Una investigación sobre el balance de agua de la cuenca atlántica del hielo patagónico y de la región de los lagos viedma y argentino. Anales de la Academia Argentina de Geografía, 3:50-92.

Heiskanen, W. A. and Moritz, H. (1967). Physical geodesy. San Francisco W. H. Freeman and Company.

Horn, B. K. (1981). Hill shading and the reflectance map. Proceedings of the IEEE, 69(1):14-47.

Hu, Y. and Freymueller, J. T. (2019). Geodetic observations of time-variable glacial isostatic adjustment in southeast alaska and its implications for earth rheology. Journal of Geophysical Research: Solid Earth, 124(9):9870-9889.

Instituto Geográfico Nacional de la República Argentina (2016). Ign. https://www .ign. gob.ar/. Online; último acceso, enero 2020. 
Ivins, E. R. and James, T. S. (1999). Simple models for late Holocene and present-day Patagonian glacier fluctuations and predictions of a geodetically detectable isostatic response. Geophysical Journal International, 138(3):601-624.

Ivins, E. R. and James, T. S. (2004). Bedrock response to llanquihue holocene and present-day glaciation in southernmost south america. Geophysical Research Letters, $31(24)$.

Ivins, E. R. and Sammis, C. G. (1996). Transient creep of a composite lower crust: 1. constitutive theory. Journal of Geophysical Research: Solid Earth, 101(B12):2798128004 .

Klemann, V., Ivins, E. R., Martinec, Z., and Wolf, D. (2007). Models of active glacial isostasy roofing warm subduction: Case of the south patagonian ice field. Journal of Geophysical Research: Solid Earth, 112(B9).

Klügel, T. and Wziontek, H. (2009). Correcting gravimeters and tiltmeters for atmospheric mass attraction using operational weather models. Journal of Geodynamics, 48(3):204 - 210. New Challenges in Earth's Dynamics - Proceedings of the 16th International Symposium on Earth Tides.

Lambeck, K. (1988). Geophysical geodesy - the slow deformations of the earth. Research supported by CNES and Universite de Paris VI. Oxford and New York, Oxford University Press, 1988, $727 \mathrm{p}$.

Lange, H., Casassa, G., Ivins, E. R., Schröder, L., Fritsche, M., Richter, A., Groh, A., and Dietrich, R. (2014). Observed crustal uplift near the southern patagonian icefield constrains improved viscoelastic earth models. Geophysical Research Letters, 41(3):805812.

Larsen, C. F., Motyka, R. J., Freymueller, J. T., Echelmeyer, K. A., and Ivins, E. R. (2005). Rapid viscoelastic uplift in southeast alaska caused by post-little ice age glacial retreat. Earth and Planetary Science Letters, 237(3):548-560.

Lin, S.-C. (2014). Three-dimensional mantle circulations and lateral slab deformation in the southern chilean subduction zone. Journal of Geophysical Research: Solid Earth, 119(4):3879-3896.

Liss, V. C. (1970). Der morenogletscher in der patagonischen kordillere: sein ungewohnliches verhalten seit 1899 und der eisdamm durchbruch des jahres 1966. Zeitschrift fur Gletscherkunde und Glazialgeologie, pages 161-180.

Lodolo, E., Menichetti, M., Bartole, R., Ben-Avraham, Z., Tassone, A., and Lippai, H. (2003). Magallanes-fagnano continental transform fault (tierra del fuego, southernmost south america). Tectonics, 22(6).

Longman, I. M. (1962). A green's function for determining the deformation of the earth under surface mass loads, 1, theory. Journal of Geophysical Research, 67:845-850. 
Longman, I. M. (1963). A green's function for determining the deformation of the earth under surface mass loads: 2. computations and numerical results. Journal of Geophysical Research, 68(2):485-496.

Love, A. E. H. (1911). Chapter IV: General theory of earth tides. Some problems of geodynamics, 49-5\%. University Press, Cambridge.

Malz, P., Meier, W., Casassa, G., Jaña, R., Skvarca, P., and Braun, M. H. (2018). Elevation and mass changes of the southern patagonia icefield derived from tandem-x and srtm data. Remote Sensing, 10(2).

Marderwald, E., Aragón Paz, J. M., Richter, A., Busch, P., Connon, G., Mendoza, L., Hormaechea, J. L., Horwath, M., and Dietrich, R. (2018a). Hydrological unloading after perito moreno glacier dam rupture: Gnss observation vs. modelling. In $E G U$ General Assembly Conference Abstracts, volume 20, page 793.

Marderwald, E., Busch, P., Aragón, J., Paz, L. M., Strelin, J., Connon, G., Hormaechea, J., Horwath, M., Scheinert, M., Dietrich, R., et al. (2018b). Paleo-lacustrine terraces of lago argentino-witnesses of crustal deformation at the patagonian icefields since late pleistocene? In Berichte zur Polar-und Meeresforschung = Reports on polar and marine research, page 123 .

Marderwald, E. R. (2014). Modelado de las mareas de grandes lagos patagónicos. Tesis de grado en Geofísica, Universidad Nacional de La Plata, AR.

Massabie, A. C., Capdevila, E. O., O., C. R., Barletta, R. H., and A, P. N. (2007). Estudios Geologicos y Geofisicos para proyectos de presas en Condor Cliff y La Barrancosa, Santa Cruz, Argentina.

Mendoza, L., Richter, A., Fritsche, M., Hormaechea, J., Perdomo, R., and Dietrich, R. (2015). Block modeling of crustal deformation in tierra del fuego from gnss velocities. Tectonophysics, 651-652:58-65.

Menichetti, M., Lodolo, E., and Tassone, A. (2008). Structural geology of the fuegian andes and magallanes fold-and-thrust belt-tierra del fuego island. Geologica Acta, 6(1):19-42.

Mercer, J. (1976). Glacial history of southernmost south america. Quaternary Research, 6(2):125-166.

Mercer, J. H. (1967). Southern Hemisphere glacier atlas. US Army Natick Laboratories, Earth Sciences Laboratory.

Milne, G. A., Davis, J. L., Mitrovica, J. X., Scherneck, H.-G., Johansson, J. M., Vermeer, M., and Koivula, H. (2001). Space-geodetic constraints on glacial isostatic adjustment in fennoscandia. Science, 291(5512):2381-2385.

Naruse, R. and Aniya, M. (1992). Outline of glacier research project in patagonia, 1990. Bulletin of Glacier Research, I(10):31-38. 
Olivero, E. B. and Martinioni, D. R. (2001). A review of the geology of the argentinian fuegian andes. Journal of South American Earth Sciences, 14(2):175 - 188. Mesozoic Palaeontology and Stratigraphy of South America and the South Atlantic.

Paruelo, J. M., Beltrán, A., Jobbágy, E., Sala, O. E., and Golluscio, R. A. (1998). The climate of patagonia: general patterns and controls on biotic processes. Ecología Austral, 8(02):085-101.

Pekeris, C. and Jarosch, H. (1958). The free oscillations of the earth. contributions in geophysics in honor of beno gutenberg.

Pelayo, A. M. and Wiens, D. A. (1989). Seismotectonics and relative plate motions in the scotia sea region. Journal of Geophysical Research: Solid Earth, 94(B6):7293-7320.

Peltier, W. R. (1998). Postglacial variations in the level of the sea: Implications for climate dynamics and solid-earth geophysics. Reviews of Geophysics, 36(4):603-689.

Peltier, W. R. and Andrews, J. T. (1976). Glacial-Isostatic Adjustment-I. The Forward Problem. Geophysical Journal International, 46(3):605-646.

Penna, N. T. and Stewart, M. P. (2003). Aliased tidal signatures in continuous gps height time series. Geophysical Research Letters, 30(23).

Reager, J. T., Gardner, A. S., Famiglietti, J. S., Wiese, D. N., Eicker, A., and Lo, M.H. (2016). A decade of sea level rise slowed by climate-driven hydrology. Science, 351(6274):699-703.

Richter, A., Groh, A., Horwath, M., Ivins, E., Marderwald, E., Hormaechea, J. L., Perdomo, R., and Dietrich, R. (2019). The rapid and steady mass loss of the patagonian icefields throughout the grace era: 2002-2017. Remote Sensing, 11(8).

Richter, A., Hormaechea, J. L., Dietrich, R., Perdomo, R., Fritsche, M., Del Cogliano, D., Liebsch, G., and Mendoza, L. (2009). Anomalous ocean load tide signal observed in lake-level variations in tierra del fuego. Geophysical Research Letters, 36(5).

Richter, A., Ivins, E., Lange, H., Mendoza, L., Schröder, L., Hormaechea, J., Casassa, G., Marderwald, E., Fritsche, M., Perdomo, R., Horwath, M., and Dietrich, R. (2016). Crustal deformation across the southern patagonian icefield observed by gnss. Earth and Planetary Science Letters, 452:206-215.

Richter, A., Mendoza, L., Perdomo, R., Hormaechea, J. L., Savcenko, R., Bosch, W., and Dietrich, R. (2012a). Pressure tide gauge records from the atlantic shelf off tierra del fuego, southernmost south america. Continental Shelf Research, 42:20-29.

Richter, A., Mendoza, L., Perdomo, R., Hormaechea, J. L., Savcenko, R., Bosch, W., and Dietrich, R. (2012b). Pressure tide gauge records from the atlantic shelf off tierra del fuego, southernmost south america. Continental Shelf Research, 42:20 - 29.

Rignot, E., Rivera, A., and Casassa, G. (2003). Contribution of the patagonia icefields of south america to sea level rise. Science, 302(5644):434-437. 
Roig, F. A. (1998). La vegetación de la patagonia. Flora patagónica, 1:48-166.

Russo, R. M., VanDecar, J. C., Comte, D., Mocanu, V. I., Gallego, A., and Murdie, R. E. (2010). Subduction of the chile ridge: Upper mantle structure and flow. Gsa Today, 20(9):4-10.

Saraceno, M., D'Onofrio, E., Fiore, M., and Grismeyer, W. (2010). Tide model comparison over the southwestern atlantic shelf. Continental Shelf Research, 30(17):1865 - 1875.

Sasagawa, G. S., Klopping, F., Niebauer, T. M., Faller, J. E., and Hilt, R. L. (1995). Intracomparison tests of the fg5 absolute gravity meters. Geophysical Research Letters, 22(4):461-464.

Savcenko, R., Bosch, W., Dettmering, D., and Seitz, F. (2012). EOT11a - Global Empirical Ocean Tide model from multi-mission satellite altimetry, with links to model results. Supplement to: Savcenko, Roman; Bosch, Wolfgang (2012): EOT11a - Empirical Ocean Tide Model from Multi-Mission Satellite Altimetry. Deutsches Geodätisches Forschungsinstitut (DGFI), München, 89, 49 pp, hdl:10013/epic.43894.d001.

Scheinert, M. and Rulke, A. (2018). Gravimetric determination of the solid-earth response to ice-mass changes in southern patagonia. Research project proposal to the German Research Foundation DFG.

Scherneck, H.-G. (1990). Loading green's functions for a continental shield with qstructure for the mantle and density constraints from the geoid. Marées terrestres Bulletin d'informations, 108:7775-7792.

Scherneck, H.-G. (1991). A parametrized solid earth tide model and ocean tide loading effects for global geodetic baseline measurements. Geophysical Journal International, 106(3):677-694.

Servicio Meteorológico Nacional (2014). Smn. https : //www .smn.gob .ar/. Online; último acceso, enero 2020.

Sood, A. and Smakhtin, V. (2015). Global hydrological models: a review. Hydrological Sciences Journal, 60(4):549-565.

Stewart, M. P., Penna, N. T., and Lichti, D. D. (2005). Investigating the propagation mechanism of unmodelled systematic errors on coordinate time series estimated using least squares. Journal of Geodesy, 79(8):479-489.

Strelin, J., Denton, G., Vandergoes, M., Ninnemann, U., and Putnam, A. (2011). Radiocarbon chronology of the late-glacial puerto bandera moraines, southern patagonian icefield, argentina. Quaternary Science Reviews, 30(19):2551-2569.

Strelin, J. A., Kaplan, M. R., Vandergoes, M. J., Denton, G. H., and Schaefer, J. M. (2014). Holocene glacier history of the lago argentino basin, southern patagonian icefield. Quaternary Science Reviews, 101:124-145. 
Stuefer, M. (1999). Investigations on mass balance and dynamics of Moreno Glacier based on field measurements and satellite imagery (Ph. D. thesis, University of Innsbruck, Austria). na.

Tregoning, P. and van Dam, T. (2005). Atmospheric pressure loading corrections applied to gps data at the observation level. Geophysical Research Letters, 32(22).

UNAVCO (2020). https://www. unavco.org/. Online; último acceso, enero 2020.

USGS (2016). Shuttle Radar Topography Mission (SRTM). SRTM 1 arc-second global. U.S. Geological Survey; https://www.usgs.gov/centers/eros.

van Dam, T., Wahr, J., and Lavallée, D. (2007). A comparison of annual vertical crustal displacements from gps and gravity recovery and climate experiment (grace) over europe. Journal of Geophysical Research: Solid Earth, 112(B3).

Wang, H., Xiang, L., Jia, L., Jiang, L., Wang, Z., Hu, B., and Gao, P. (2012). Load love numbers and green's functions for elastic earth models prem, iasp91, ak135, and modified models with refined crustal structure from crust 2.0. Computers and Geosciences, 49:190 - 199 .

Warren, C. and Aniya, M. (1999). The calving glaciers of southern south america. Global and Planetary Change, 22(1):59 - 77.

Webb, D. (1976). A model of continental-shelf resonances. Deep Sea Research and Oceanographic Abstracts, 23(1):1 - 15 .

Wessel, P., Smith, W. H. F., Scharroo, R., Luis, J., and Wobbe, F. (2013). Generic mapping tools: Improved version released. Eos, Transactions American Geophysical Union, 94(45):409-410.

Whitehouse, P. L. (2018). Glacial isostatic adjustment modelling: historical perspectives, recent advances, and future directions. Earth Surface Dynamics, 6(2):401-429.

Wiens, D., Magnani, B., Ito, S., Wickert, A., Noren, A., and Ivins, E. (2017). Collaborative research: Solid earth response of the patagonian andes to post-little ice age glacial retreat. National Science Foundation Project.

Willis, M. J., Melkonian, A. K., Pritchard, M. E., and Ramage, J. M. (2012a). Ice loss rates at the northern patagonian icefield derived using a decade of satellite remote sensing. Remote Sensing of Environment, 117:184-198. Remote Sensing of Urban Environments.

Willis, M. J., Melkonian, A. K., Pritchard, M. E., and Rivera, A. (2012b). Ice loss from the southern patagonian ice field, south america, between 2000 and 2012. Geophysical Research Letters, 39(17).

Woodhouse, J. and Deuss, A. (2015). 1.03 - theory and observations - earth's free oscillations. In Schubert, G., editor, Treatise on Geophysics (Second Edition), pages 79 115. Elsevier, Oxford, second edition. 